CERN-TH-7528/95

IASSNS-HEP-94/106

OSU-M-94-3

hep-th/9506091

\title{
Mirror Symmetry and the Moduli Space for Generic Hypersurfaces in Toric Varieties
}

\author{
Per Berglund \\ School of Natural Sciences \\ Institute for Advanced Study \\ Princeton, NJ 08540, USA \\ Sheldon Katz \\ Department of Mathematics \\ Oklahoma State University \\ Stillwater, OK 74078, USA \\ and \\ Albrecht Klemm \\ Theory Division, CERN \\ CH-1211 Geneva 23, Switzerland
}

The moduli dependence of $(2,2)$ superstring compactifications based on CalabiYau hypersurfaces in weighted projective space has so far only been investigated for Fermat-type polynomial constraints. These correspond to Landau-Ginzburg orbifolds with $c=9$ whose potential is a sum of $A$-type singularities. Here we consider the generalization to arbitrary quasi-homogeneous singularities at $c=9$. We use mirror symmetry to derive the dependence of the models on the complexified Kähler moduli and check the expansions of some topological correlation functions against explicit genus zero and genus one instanton calculations. As an important application we give examples of how non-algebraic ("twisted") deformations can be mapped to algebraic ones, hence allowing us to study the full moduli space. We also study how moduli spaces can be nested in each other, thus enabling a (singular) transition from one theory to another. Following the recent work of Greene, Morrison and Strominger we show that this corresponds to black hole condensation in type II string theories compactified on Calabi-Yau manifolds.

CERN-TH-7528/95

$6 / 95$

Email: berglund@guinness.ias.edu, katz@math.okstate.edu, klemm@nxth04.cern.ch 


\section{Motivation and Outline of the Strategy}

The study of the moduli dependence of two-dimensional conformal field theories is essential for understanding the symmetries and the vacuum structure of the critical string. For conformal field theories with extended $N=2$ superconformal symmetry remarkable progress in this question was made after realizing the similarity of this problem with the geometrical problem of the variation of the complex structure [1]. This implies that the (topological) correlation functions are related to solutions of Fuchsian differential equations and have natural modular properties with respect to the four-dimensional spacetime moduli [2]. Closed string compactifications with $N=1$ spacetime supersymmetry actually require an extension of the conformal symmetry to a global $N=2$ superconformal symmetry for the right moving modes on the worldsheet; heterotic compactifications with $E_{8} \times E_{6}$ gauge group are based on a left-right symmetric $(N, \bar{N})=(2,2)$ superconformal internal sectors with $c=\bar{c}=9$. The truly marginal operators, which preserve the $(2,2)$ structure, in these phenomenologically motivated string compactifications come in two equivalent types related to the left-right chiral $(c, c)$ states and the left anti-chiral, right chiral $(a, c)$ states of the $(2,2)$ theory [3] 1 . The two types of states form two rings whose structure constants depend only on one type of moduli respectively; our convention will be to identify the $(c, c)$ ring with the complex structure deformations (also known as the B-model in the language of topological field theory [5]) and the $(a, c)$ ring with deformations of the complexified Kähler structure (the A-model in the topological sigma model) of the target space $X$, a Calabi-Yau threefold.

Unlike the dependence of the theory on the complexified Kähler structure, which contains the information about the holomorphic instantons on $X$, the geometrical problem of complex structure deformations is a well studied subject in classical geometry [6]. This fact and the equivalent structure of the two rings from the point of view of the $(2,2)$ theory, which is the origin of mirror symmetry [3:7], has motivated the key trick to solve both sectors; to find a geometrical object $X^{*}$ yielding the identical $(2,2)$ theory in such a way that the deformations of the complexified Kähler structure on $X\left(X^{*}\right)$ can be identified with the complex structure deformations on $X^{*}(X)$. If this holds for $X$ and $X^{*}$, the two manifolds form a so called mirror pair.

Before going on let us briefly recall the phenomenological implications of the above. The physical (normalized) Yukawa couplings can in principle be computed since we know

1 These marginal operators are in one to one correspondence with the $\mathbf{2 7}$ and $\overline{\mathbf{2 7}}$ of $E_{6}$. There is yet a third class, namely the marginal operators which correspond to $\mathbf{1}$. They could potentially enlarge the set of moduli fields. They correspond to deformations of the tangent bundle of the Calabi-Yau manifold in question, $X$, and are given by $H^{1}\left(X, E n d T_{X}\right)$. We will focus our attention to the traditional space of $(2,2)$ preserving deformations. However, see [4] for some recent work. 
the metric on the moduli space for both the complex structure and the Kähler structure deformations; the latter thanks to mirror symmetry. In addition, as was first pointed out by Bershadsky et. al. [8] and recently amplified by Kaplunovsky and Louis [9], threshold corrections to gauge couplings in the four-dimensional effective field theory can be inferred from the detailed knowledge of the singularity structure of the moduli space.

Recently it has become clear that the moduli spaces of Calabi-Yau manifolds might play an essential rôle already when writing down a consistent four dimensional $N=2$ supergravity theory, independently of whether one believes that it arises as a low energy effective theory from string compactification or not. In the work of Seiberg and Witten [10] the moduli space of four dimensional pure $S U(2)$ Super-Yang-Mills theory with global $N=2$ supersymmetry is governed by rigid special geometry, and due to the consistency condition of the positive kinetic terms uniquely it can be identified with the moduli space of a torus2. Similarly, the moduli space of $N=2$ supergravity is known to exhibit nonrigid special geometry [13] and a natural geometrical object associated to this structure is a Calabi-Yau threefold. Indeed, in a recent paper, Kachru and Vafa [14 give examples of heterotic stringy realizations of [10].

The classification of $N=2$ SCFT with $c<3$ follows an A-D-E scheme which has, via the Landau-Ginzburg (LG) approach [15], a beautiful relation to the singularities of modality zero [16]. Superstring compactifications with $N=1$ spacetime supersymmetry can be constructed by taking suitable tensor products of these models such that $c=9$, adding free theories for the uncompactified spacetime degrees of freedom including the gauge degrees of freedom in the left-moving sector, and implementing a generalized GSOprojection [17].

The program for solving the full moduli dependence of the $(2,2)$ theory and checking the instanton predictions has so far been studied only for theories based on tensor products of the $A$-series [18, 19,20,21,22]. (For a rather different approach than the one pursued here, using the linear sigma model, see [23], following the original mirror symmetry construction by Greene and Plesser [24].) Geometrically they can be identified with hypersurfaces of Fermat-type in weighted projective spaces $X=\left\{\vec{x} \in \mathbf{P}^{4}(\vec{w}) \mid \sum_{i=1}^{5} x_{i}^{n_{i}}=0\right\}$. These cases are however only a very tiny subset of all transversal quasi-homogeneous singularities or Landau-Ginzburg potentials with singularity index $\beta=3 / 2$, which correspond to rational $N=2$ SCFT with $c=9$. Theories of this general type have been classified in [25]. Here we develop the methods to treat these generic quasi-homogeneous potentials involving arbitrary combinations of $A-D-E$ invariants as well as new types of singularities.

2 The positivity condition is solved in this approach by the second Riemann inequality for the period lattice. In general, from lattices of dimension six on, not every such such structure, which defines an abelian variety, comes from a geometric curve; this is known as Schottky problem. Families of curves however that generalize[10] for the $S U(N)$ series have been identified [11, 12]. 
Already the first step, to find the geometrical object $X^{*}$, is more tricky for the general case. For models with $A, D_{k}, k \leq 3$ and/or $E$ invariants one has always the simplification, that the complex structure moduli space of $X^{*}$ is the restriction of the moduli space of $X$ to the invariant subsector with respect to a discrete group $H$; in other words $X^{*} \simeq X / H$ (modulo desingularizations). Therefore in these cases one in effect only needs to consider the restricted complex structure deformation of the original manifold $X$ itself. The general case, however, requires the construction of a new variety. The following two approaches to that problem will become relevant for our calculation.

i) For models which admit a description in terms of Fermat-type polynomials Batyrev has suggested a method of constructing the pair $X$ and $X^{*}$ as hypersurfaces in toric varieties defined by a pair of reflexive simplices [26,27], see also [28]. It was later noticed [22,29, 30 that this method applies also to general quasi-homogeneous hypersurfaces and can be used to construct all the mirror manifolds for the hypersurfaces in $\mathbf{P}^{4}(\vec{w})$ which where classified in [25].

ii) An alternative approach [31,32] starts from the following symmetry consideration. The LG-theory $\mathcal{P}$ is defined by a transversal potential $p\left(x_{1}, \ldots, x_{5}\right)$ quasi-homogeneous of degree $d$ with respect to the weights $w_{i}$, i.e. $p\left(\lambda^{w_{1}} x_{1}, \ldots, \lambda^{w_{5}} x_{5}\right)=\lambda^{d} p\left(x_{1}, \ldots, x_{5}\right)$. The potential has an invariance group $\mathcal{G}(\mathcal{P})$ whose elements $\gamma$ act on the coordinates by phase multiplication $x_{i} \rightarrow x_{i} \exp \left(\gamma_{i}\right)$. The GSO-projection onto integral charge states is implemented in the internal sector by orbifoldization with respect to a subgroup $\mathbb{Z}_{d} \in \mathcal{G}(\mathcal{P})$ acting by $x_{i} \rightarrow x_{i} \exp \left(2 \pi i \frac{w_{i}}{d}\right)$ [17]. The string compactification based on the internal sector $\mathcal{P} / \mathbb{Z}_{d}$ has a simple geometrical interpretation. Namely the compact part of the target space is given by $X=\left\{\vec{x} \in \mathbf{P}^{4}(\vec{w}) \mid p\left(x_{1}, \ldots, x_{5}\right)=0\right\}$. Any orbifold with respect to a group $H$ with $\mathbb{Z}_{d} \subset H \subset \mathcal{G}(\mathcal{P})$ and $\prod_{i=1}^{5} \exp \left(\kappa_{i}\right)=1$ for all $\kappa \in H$, leads likewise to a consistent string compactification. The compact part of the target space is now $X /\left(H / \mathbb{Z}_{d}\right)$. It now follows from general arguments [33] that the orbifold $\mathcal{O}$ with respect to an abelian group $H$, will have a dual symmetry group $\mathcal{G}_{q}(\mathcal{O})$ called the quantum symmetry, which is isomorphic to $H$ and manifest in the operator algebra of the twisted states of $\mathcal{O}$. In the case of the above orbifold $\mathcal{O}=\mathcal{P} / \mathbb{Z}_{d}$ all $(a, c)$ states belong to the twisted states and $\mathcal{G}_{q}(\mathcal{O}) \cong \mathbb{Z}_{d}$. On the other hand the operator algebra of the invariant $(c, c)$ states is determined by $\mathcal{G}_{g}(\mathcal{O})=\mathcal{G}(\mathcal{P}) / \mathbb{Z}_{d}$, the so called geometrical symmetry group. To exchange the rôle of the $(c, c)$ and the $(a, c)$ ring and to construct a mirror pair one therefore tries to construct two orbifolds $\mathcal{O}$ and $\mathcal{O}^{*}$ in which the geometrical symmetry and the quantum symmetry are exchanged, i.e. $\mathcal{G}_{g}(\mathcal{O}) \cong \mathcal{G}_{q}\left(\mathcal{O}^{*}\right)$ and $\mathcal{G}_{q}(\mathcal{O}) \cong \mathcal{G}_{g}\left(\mathcal{O}^{*}\right)$. As was recognized in [31] $N=2$ models based on tensor products of minimal models always have a symmetry group $H$ with $\mathbb{Z}_{d} \subset H \subset \mathcal{G}(\mathcal{P})$ such that $\tilde{\mathbb{Z}}_{d}=\mathcal{G}(\mathcal{P}) / H$ and the quantum symmetry and the geometrical symmetry are in fact exchanged for the pair $\mathcal{O}=\mathcal{P} / \mathbb{Z}_{d}, \mathcal{O}^{*}=\mathcal{P} / H$. 
For general LG-models such an $H$ need not exist. In [32] the authors present a generalization 3 of the argument of [31] and consider a LG-potentials $p\left(x_{1}, \ldots, x_{r}\right)$, which is transversal for a polynomial configuration with $r$ monomials, i.e. $p=\sum_{i=1}^{r} X^{v^{(i)}}$ with $v^{(i)}$ vectors of exponents and $X^{v^{(i)}}:=x_{1}^{v_{1}^{(i)}} \cdots x_{r}^{v_{r}^{(i)}}, v_{j}^{(i)} \in \mathbf{N}_{0}$. Then one can consider the "transposed" polynomial $\hat{p}=\sum_{i=1}^{r} Y^{\hat{v}^{(i)}}$, where the new exponent vectors $\hat{v}^{(i)}$ are defined by $\hat{v}_{r}^{(i)}=v_{i}^{(r)}$. It follows from the transversality condition [25] that all monomials $X^{v^{(i)}}$ in $p$ as well as the transposed monomials in $\hat{p}$ have the form $x_{i}^{n_{i}} x_{j}$, with $i, j$ not necessarily different. Using this one can see that $\mathcal{G}(\mathcal{P}) \cong \mathcal{G}(\hat{\mathcal{P}})$ and it was argued in 32] that there exists a group $H$ with $\mathbb{Z}_{\hat{d}} \subset H \subset \mathcal{G}(\mathcal{P})$ such that $\mathcal{G}_{q}\left(\mathcal{P} / \mathbb{Z}_{d}\right) \cong \mathcal{G}_{g}(\hat{\mathcal{P}} / H)$ and $\mathcal{G}_{g}\left(\mathcal{P} / \mathbb{Z}_{d}\right) \cong \mathcal{G}_{q}(\hat{\mathcal{P}} / H)$. In fact the transposition rule also holds for many non-transverse polynomials [30], where it was also shown to be consistent with approach i).

In section 2 we will briefly review the construction of Calabi-Yau hypersurfaces in toric varieties, heavily relying on the methods introduced in [22]. In particular we will introduce the Batyrev-Cox homogeneous coordinate ring [37] which will simplify the construction of the period vector as well as the Picard-Fuchs equation associated to it. To generalize this approach to cases which have no ordinary LG-description, i.e. where no description as hypersurface in $\mathbf{P}^{4}(\vec{w})$ or orbifolds thereof is available, we develop methods to derive the Picard-Fuchs equations directly from the combinatorial data of the dual polyhedron $\Delta^{*}$.

In section 3.1 we then consider generalized hypersurfaces in $\mathbf{P}^{4}(\vec{w})$ with two Kähler moduli. We use the constructions $(i),(i i)$ to derive the Picard-Fuchs equations for the period integrals in order to study the complex structure deformations of $X^{*}$. It is convenient to follow first (ii) and use $\hat{\mathcal{P}} / H$ as a representation of $X^{*}$. Then one can apply the standard Dwork-Katz-Griffiths [38,39,40] reduction formulas adapted to weighted projective spaces for the derivation of the differential equations. From this information we calculate the number of holomorphic instantons of genus zero and genus one on $X$. A detailed check of these predictions will be presented in the section 4, where we also note that the discrepancy between the Mori cones affects the nature of the large complex structure limit, but does not affect the validity of the instanton expansions.

The construction of [32] allows one to obtain $X^{*}$ for the majority of the LG-potentials in [25]. It fails however for LG-potentials for which a transversal configuration requires

3 Contrary to the original mirror symmetry construction [24], there is no supporting argument at the level of the underlying conformal field theory, since the exact $N=2$ SCFT theory is not known. However, the calculation here, the affirmative check of the elliptic genus [34] as well as the chiral ring [35] suggests that this is true. Moreover, Morrison and Plesser have a promising approach to providing such an argument.[36]

4 As usual, the Mori cone of the associated toric variety enters in a crucial way. We observe that the Mori cone of the Calabi-Yau hypersurface may be strictly smaller than the Mori cone of the toric variety. 
more than $r$ monomials; in that case, Batyrev's approach will apply, as shown in [22,30]. We consider therefore in section $3.2 \mathrm{such}$ a case. It turns out that if we restrict to a suitable non-transversal configuration involving $r$ terms (where $r=4+1$ in our case since the hypersurfaces are embedded in a toric variety of dimension four) and consider the transposed polynomial $\hat{P} / H$ as before, we get an orbifold of a non-transversal hypersurface $X^{*}$ in $\mathbf{P}^{4}(\vec{w})$, which can be conveniently used to derive the set of differential equations of Fuchsian type. The latter reproduce the topological couplings and at least to lowest order the genus zero instantons of $X$, see section 4 . This approach can be justified by translating the operator identities, which hold modulo the ideal of $\hat{P}$ (equations of motions), to the Laurent-monomials and derivatives of the Laurent-polynomial and using the partial differentiation rule of section (3.1).

In section 5 we apply the techniques developed in the earlier sections to study some new phenomena. Given a toric variety based on a polyhedron $\Delta$, we construct new reflexive polyhedra. In 5.1 we give examples of reflexive polyhedra, which are not associated to hypersurfaces in $\mathbf{P}^{4}(\vec{w})$ and apply the methods outlined in section 2 to get the Picard-Fuchs equations also in this case. In section 5.2, we the study the phenomenon of embedding the moduli space of one Calabi-Yau space into the moduli space of another Calabi-Yau space and develop a quite general strategy for constructing an algebraic realization of the space of deformations (section 5.3). The latter approach removes, what is sometimes described as the "twisted sector problem" in the physics literature. Finally, in section 6 we will discuss the general validity of the computation and give an algorithm for which, in principle, a model with any number of moduli can be solved. We also briefly comment on the connection with the recent developments in type II string theory compactified on Calabi-Yau manifolds.

\section{General construction of Picard-Fuchs equations for Calabi-Yau hypersur- faces in toric varieties}

Let us start by giving a brief review of the existing methods by which the the PicardFuchs equations are obtained. For more details, see [22].

Given a weighted projective space $\mathbf{P}^{4}(\vec{w})$ we construct the Newton polyhedron, $\Delta$, as the convex hull (shifted by $(-1,-1,-1,-1,-1)$ ) of the most general polynomial $p$ of degree $d=\sum_{i=1}^{5} w_{i}$

$$
\Delta=\operatorname{Conv}\left(\left\{n \in \mathbb{Z}^{5} \mid \sum_{i=1}^{5} n_{i} w_{i}=0, n_{i} \geq-1 \forall i\right\}\right),
$$

which lies in a hyperplane in $\mathbb{R}^{5}$ passing through the origin. For any set of weights which admits a transverse polynomial, it has been shown in [30 that the Newton polyhedron is 
reflexive, yielding a toric variety birational to $\mathbf{P}^{4}(\vec{w})$. (Reflexivity in these cases has been checked independently by the third author.) The polar polyhedron, $\Delta^{*}$, is given by

$$
\Delta^{*}=\operatorname{Conv}\left(\left\{m \in \Lambda^{*} \mid<n, m>\geq-1, \quad \forall n \in \Delta\right\}\right)
$$

where $\Lambda^{*}$ is the dual lattice to $\Lambda=\left\{n \in \mathbb{Z}^{5} \mid \sum_{i=1}^{5} n_{i} w_{i}=0\right\}$. We can identify five vertices, $v^{*(i)}, i=1, \ldots, 5$ satisfying the linear relation [29]

$$
\sum_{i=1}^{5} k_{i} \cdot v^{*(i)}=0 \text {. }
$$

When $k_{1}=1$ we have

$$
v^{*(1)}=\left(-k_{2},-k_{3},-k_{4},-k_{5}\right), v^{*(i+1)}=e_{i}, \quad i=1, \ldots, 4
$$

where the $e_{i}$ are the standard basis elements in $\mathbb{Z}^{4}$.

We are interested in studying hyperplane sections, $X^{*}$, of the toric variety $\mathbf{P}_{\Delta^{*}}$, given by

$$
P=\sum_{i \in \Delta^{*} \cap \Lambda^{*}} a_{i} \phi_{i}=\sum_{i \in \Delta^{*} \cap \Lambda^{*}} \prod_{j} Y_{j}^{\nu_{j}^{*(i)}}
$$

The claim [26] is that hypersurfaces $X \in \mathbf{P}^{4}(\vec{w})$ and $X^{*} \in \mathbf{P}_{\Delta^{*}}$ are mirror partners. An alternative way is to specify an étale map $Y(y)$ to the homogeneous coordinates $\left(y_{1}: \ldots\right.$ : $y_{5}$ ) of a suitable four dimensional weighted projective space $\hat{\mathbf{P}}^{4}(\vec{w})$ (this map is actually only étale on the respective tori), which identifies (2.5) in $\mathbf{P}_{\Delta^{*}}$ with the hypersurface defined by an ordinary polynomial constraint $\hat{p}=\left(y_{1} y_{2} y_{3} y_{4} y_{5}\right) P(Y(y))=0$ in this $\hat{\mathbf{P}}^{4}(\vec{w})$. Note that in general $\hat{\mathbf{P}}^{4}(\vec{w}) \neq \mathbf{P}^{4}(\vec{w})$ In fact if we set $a_{i}=0, i \neq 1, \ldots, 5$ then (2.5) reduces to the transposed polynomial $\hat{p}_{0}\left(y_{i}\right)$, of $p_{0}$; here $p_{0}\left(x_{i}\right)$ defines a (possibly non-transverse) hypersurface in $\mathbf{P}^{4}(\vec{w})$, see ii) in the previous section. Note that the map $Y(y)$ is in general not one-to-one, but rather there is an automorphism which is isomorphic to $H$ the group which we need to divide by in order to make $\left\{\hat{p}\left(y_{i}\right)=0\right\} / H$ the mirror of a hypersurface in $\mathbf{P}^{4}(\vec{w})$ described by $p=0$. (For a more detailed account of the correspondence between the toric construction and the transposition scheme, see [29,30].)

Yet a third way of identifying the hyperplane section in $\mathbf{P}_{\Delta^{*}}$ is to use the homogeneous coordinate ring defined by Batyrev and Cox [37. To each of the vertices, $\nu^{(i)}$ in $\Delta$ we associate a coordinate $x_{i}$. As for a homogeneous coordinate in a weighted projective space, each $x_{i}$ has a weight, or rather a multiple weight, given by positive linear relations among the $\nu^{(i)}$. However, for our purposes it is enough to note that to each vertex in the polar polytope, $\Delta^{*}$, corresponds a monomial $\phi_{j}$ in terms of the $x_{i}$;

$$
\phi_{j}=\sum_{i \in \Lambda \cap \Delta}<\left(v^{(i)}, 1\right),\left(v^{(j) *}, 1\right)>
$$


Thus, $P=\sum_{j \in \Lambda^{*} \cap \Delta^{*}} a_{i} \phi_{i}$ defines a hyperplanes section in $\mathbf{P}_{\Delta^{*}}$. Note that if we were to restrict to a particular set of five vertices in $\Delta$ one can show $P$ reduces to $\hat{p}$ defined above through the étale map [29,30. In the examples we will mostly be using the second and third representation, but that is only as a matter of convenience; all results can be derived using Batyrev's original set of coordinates (2.5).

The next step is to find the generators of the Mori cone as they are relevant in studying the large complex structure limit; recall that the Mori cone is dual to the Kähler cone. Given $\Delta^{*}$ we have to specify a particular triangulation; more precisely a star subdivision of $\Delta^{*}$ from the interior point $\nu^{*(0)}$. In general this triangulation is not unique. In particular there may exist more than one subdivision which admits a Kähler resolution, i.e. there is more than one Calabi-Yau phase [41]; see section 3 for examples.

Once a particular subdivision is picked an algorithm for constructing the generators of the Mori cone is as follows:

i) Extend $\nu^{*(i)}$ to $\bar{\nu}^{*(i)}=\left(1, \nu^{*(i)}\right)$

ii) Consider every pair $\left(S_{k}, S_{l}\right)$ of four-dimensional simplices in the star subdivision of $\Delta^{*}$ which have a common three-dimensional simplex $s_{i}=S_{k} \cap S_{l}$.

iii) Find for all such pairs the unique linear relation $\sum_{i=1}^{6} l_{i}^{(k, l)} \bar{\nu}^{*(i)}=0$ among the six points $\nu^{*(i)}$ of $S_{l} \cup S_{k}$ in which the $l_{i}^{(k, l)}$ are minimal integers and the coefficients of the two points in $\left(S_{k} \cup S_{l}\right) \backslash\left(S_{l} \cap S_{k}\right)$ are non-negative.

iv) Find the minimal integer vectors $l^{(i)}$ by which every $l^{(k, l)}$ can be expressed as positive integer linear combination. These are the generators of the Mori cone.

Next we derive the Picard-Fuchs equation for $X^{*}$ from the residue expression for the periods. There are two residue forms for the periods; the Laurent polynomial (2.5) or the transposed polynomial from the étale map, both of which we will use for the derivation. The first one reads 27]

$$
\Pi_{i}\left(a_{0}, \ldots, a_{p}\right)=\int_{\Gamma_{i}} \frac{\omega}{P}, \quad i=1, \ldots, 2\left(h^{2,1}+1\right),
$$

where $\Gamma_{i} \in H^{4}(\mathbf{T} \backslash X)$ ( $\mathbf{T}$ is the algebraic torus associated to the toric variety [27]), and $\omega=\frac{d Y_{1}}{Y_{1}} \wedge \frac{d Y_{2}}{Y_{2}} \wedge \frac{d Y_{3}}{Y_{3}} \wedge \frac{d Y_{4}}{Y_{4}}$. Alternatively, we can write the following expression for the periods in terms of the transposed polynomial $\hat{p}=0$, 40

$$
\hat{\Pi}_{i}\left(a_{0}, \ldots, a_{p}\right)=\int_{\gamma} \int_{\hat{\Gamma}_{i}} \frac{\hat{\omega}}{\hat{p}}, \quad i=1, \ldots, 2\left(h^{2,1}+1\right) .
$$

5 This algorithm is equivalent to the one described by Oda and Park [42, but is simpler to apply. 
Here $\hat{\omega}=\sum_{i=1}^{5}(-1)^{i} w_{i} y_{i} d y_{1} \wedge \ldots \wedge \widehat{d y}_{i} \wedge \ldots \wedge d y_{5} ; \hat{\Gamma}_{i}$ is an element of $H_{3}(\hat{X}, \mathbb{Z})$ and $\gamma$ a small curve around $\hat{p}=0$ in the 4 -dimensional embedding space. Note that by performing a change of variables as dictated by the étale map one can show that (2.7) is equivalent to (2.8). Symmetry considerations now make the derivation of the Picard-Fuchs equations a short argument. The linear relations between the points $\bar{\nu}^{*(i)}=\left(\nu^{*(i)}, 1\right)$ in the extended dual polyhedron $\bar{\Delta}^{*}$ as expressed by the $l^{(i)}$ translates into relations among the $\phi_{i}$,

$$
\mathcal{L}_{k}\left(\phi_{i}\right)=0 .
$$

However, from the definition of $\Pi_{i}$, the (2.9) are equivalent to

$$
\mathcal{L}_{k}\left(\partial_{a_{i}}\right) \Pi_{j}=0 .
$$

Finally, due to the $\left(\mathbb{C}^{*}\right)^{5}$-invariance of $\Pi_{j}\left(a_{i}\right)$ we choose the following combinations as the coordinates relevant in the large complex structure limit [27]

$$
z_{k}=(-1)^{l_{0}^{(k)}} \prod_{i} a_{i}^{l_{i}^{(k)}} .
$$

They will lead directly to the large Kähler structure limit at $z_{k}=0$ at which the monodromy is maximal unipotent [43]. Thus, using $\theta_{i}=z_{i} \frac{d}{z_{i}}$, eq. (2.10) is readily transformed tol

$$
\mathcal{L}_{k}\left(\theta_{i}, z_{i}\right) \hat{\Pi}_{j}=0 .
$$

However, the above system (2.12) has in general more than $2\left(h^{2,1}+1\right)$ solutions as can be seen by studying the number of solutions to the indicial problem. This can be resurrected in the following two ways. On one hand, one can try to factorize the set of differential operators $\mathcal{L}_{k}$ in order to reproduce operators of lower order; for examples see [22] and section 3.1. Alternatively, we can derive the differential operators by the more standard manipulations of the residuum expressions (2.7) and (2.8). In particular, there exist relations of order $n$ based on the use of the ideal $\partial_{y_{i}} \hat{p}$, ,

$$
\Xi_{k}\left(\partial_{y_{i}} \hat{p}, \phi_{j}, y_{i}\right)=0
$$

This implies $\int_{\gamma} \int_{\Gamma_{i}} \frac{\Xi \hat{\omega}}{\hat{p}^{n+1}}=0$. We can use the partial integration rule $\frac{m(y) \partial_{y_{i}} \hat{p}}{\hat{p}^{n+1}}=\frac{1}{n} \frac{\partial_{y_{i}} m(y)}{\hat{p}^{n}}$ under the integral sign (2.8), which follows from the fact that $\partial_{y_{i}}\left(\frac{m(y)}{\hat{p}^{n}}\right) \hat{\omega}$ is exact, provided the integral over both sides of the above equation makes sense as periods, which is

${ }^{6}$ For historical reasons we rescale also the periods and use $\Pi_{i}=\frac{1}{a_{0}} \hat{\Pi}_{i}$. This brings the system of differential equations in the form, first discussed by Gelfand-Kapranov and Zelevinskii [44].

7 There are in general many ways of obtaining relations such as the one below. From a technical point of view, it is preferable to use the ideal in such away that the use of the partial integration rule becomes trivial. 
the case if $m(y) \partial_{y_{i}} \hat{p}$ is homogeneous of degree $d n$, see e.g. [45, 19]. In analogy to (2.9) and (2.10) we get a differential equation satisfied by the period vector,

$$
\hat{\mathcal{L}}_{k}\left(\theta_{i}, z_{i}\right) \hat{\Pi}_{j}=0
$$

It is clear that (2.13) can be used analogously in (2.7). To be more precise $\Theta_{i}=Y_{i} \frac{d}{d Y_{i}}$ and the partial integration rule, which due to the measure $\omega$ of $(2.7)$ now reads $8 \frac{m(Y) d_{i} P}{P^{n}}=$ $\frac{1}{n-1} \frac{\left(d_{i}+n-2\right) m(Y)}{P^{n-1}}$. Also this integration rule is valid only if the integral (2.7) over both sides makes sense as periods, which is the case if the points associated to $m(Y) d_{i} P$ are in $(n-1) \Delta^{*}$ and the ones associated to $\left(d_{i}+n-2\right) m(Y)$ are in $(n-2) \Delta^{*}$, where $k \Delta^{*}$ denotes the polyhedron $\Delta^{*}$ scaled by $k$.

It should be obvious from the first and the last derivation of the Picard-Fuchs equation, that the étale map and the transposed polynomial are auxiliary constructions. Their virtue is to introduce a suitable grading which facilitate the calculations. All relevant data however can be obtained directly from the polyhedron $\Delta^{*}$ and $(2.7)$.

Finally, from the sets of operators $\left(\mathcal{L}_{k}, \hat{\mathcal{L}}_{k}\right)$ one then has to select the set of operators $\left(L_{k}\right)$ which will reproduce the relevant ring structure. Unfortunately, we do not know of a general recipe for how this is done, and at present we have to resort to a case by case study, see section 3 .

With the relevant set of operators at hand $\left(L_{k}\right)$ we are now ready to compute the Yukawa couplings, the mirror map and then the instanton expansion. Schematically the procedure is as follows. (For more details, see [22,46].) One first finds solutions, $w_{j}$, of the Picard-Fuchs system $\left(L_{k} \hat{\Pi}=0\right)$ with maximally unipotent monodromy at $z_{i}=0$. Then the flat coordinates are given by $t_{j}=w_{j} / w_{0}$ where $w_{0}$ is the power series solution at $z_{i}=0$. From the $\left(L_{k}\right)$ one can derive linear relations among the Yukawa couplings $K_{z_{i} z_{j} z_{k}}$ and their derivatives. Thus rather than using the explicit solutions, and a knowledge of an integral symplectic basis, we derive the Yukawa couplings (up to an overall normalization) directly from the differential operators. This also gives us the discriminant locus, $\Delta$, i.e. the codimension one set where the Calabi-Yau hypersurface $X^{*}$ is singular.

From the Picard-Fuchs operators, we can also determine the intersection numbers up to a normalization as the coefficients of the unique degree three element in

$$
\mathbb{C}\left[\theta_{i}\right] /\left\{\lim _{z_{i} \rightarrow 0} L_{k}\right\}
$$

The normalization may be fixed by the intersection of

$$
K_{J_{1} J_{1} J_{1}}:=\int_{X} J_{1} \wedge J_{1} \wedge J_{1}=\left(\frac{d}{\prod_{i=1}^{5} w_{i}}\right) n_{0}^{3},
$$

8 The indexing of the $d_{i}$ is such that $d_{i} \prod_{j} Y_{j}^{\nu_{j}^{*(k)}}=\left(1-\left\langle\nu^{(i)}, \nu^{*(k)}\right\rangle\right) \prod_{j} Y_{j}^{\nu_{j}^{*(k)}}$. 
where $n_{0}$ is the least common multiple of the orders of all fixpoints in $X$. Alternatively, by considering the restriction to a one-parameter subspace of the moduli space of complex structure deformations spanned by the deformation corresponding to the interior point in $\Delta^{*}$, one can directly compute (2.15) 47].

With both the Yukawa couplings and the flat coordinates at hand it is then straightforward to map our results to that of the Yukawa coupling as a function of the Kähler moduli in $X$, the hypersurface in $\mathbf{P}^{4}(\vec{w})$. The details of this are standard and can be found in 22,46]. Here we only record the result;

$$
K_{\tilde{t}_{i} \tilde{t}_{j} \tilde{t}_{k}}(\tilde{t})=\frac{1}{w_{0}^{2}} \sum_{l, m, n} \frac{\partial x_{l}}{\partial \tilde{t}_{i}} \frac{\partial x_{m}}{\partial \tilde{t}_{j}} \frac{\partial x_{n}}{\partial \tilde{t}_{k}} K_{z_{l} z_{m} z_{n}}
$$

where the $\tilde{t}_{i}$ corresponds to an integral basis of $H^{1,1}(X, \mathbb{Z})$ and are related to the flat coordinates by an integral similarity transformation. Finally, by expanding (2.16) in terms of the variables $q_{i}=\exp \left(2 \pi i \tilde{t}_{i}\right)$, we can read off the invariants of the rational curves $N\left(\left\{n_{l}\right\}\right)$,

$$
K_{\tilde{t}_{i} \tilde{t}_{j} \tilde{t}_{k}}(\tilde{t})=K_{i j k}^{0}+\sum_{n_{i}} \frac{N\left(\left\{n_{l}\right\}\right) n_{i} n_{j} n_{k}}{1-\prod_{l} q_{l}^{n_{l}}} \prod_{l} q_{l}^{n_{l}} .
$$

It is well-known that there are no corrections to the Yukawa couplings at higher genus. Still it is interesting to note that invariants of elliptic curves (genus one) can be studied by means of the following index $F_{1}^{t o p}[8]$,

$$
F_{1}^{t o p}=\log \left[\left(\frac{1}{\omega_{0}}\right)^{5-\chi / 12} \frac{\partial\left(z_{i}\right)}{\partial\left(t_{i}\right)} f(z)\right]+\text { const. }
$$

where $f$ is a holomorphic function with singularities only at singular points on $X$ and so is related to the discriminant locus. Thus, we make the ansatz

$$
f(z)=\left(\prod_{j} \Delta_{j}^{r_{j}}\right) \prod_{i} z_{i}^{s_{i}}
$$

with the $s_{i}$ fixed by using the following asymptotic relation (valid at the large radius limit)

$$
\lim _{t, \bar{t} \rightarrow \infty} F_{1}=-\frac{2 \pi i}{12} \sum_{i}\left(t_{i}+\bar{t}_{i}\right) \int c_{2} J_{i}
$$

In (2.19) the product over the $j$ is taken over the components $\Delta_{j}$ of the discriminant locus. The $r_{j}$ are determined by knowing some of the lowest order invariants of the elliptic curves; see the examples for more details. 


\section{Examples}

\subsection{Non-Fermat hypersurfaces in $\mathbf{P}^{4}$ with two moduli}

The minimal Kähler structure moduli system of transversal non-Fermat Calabi-Yau hypersurfaces in weighted $\mathbf{P}^{4}$ is two dimensional. In Table 3.1 the complete set of these models [25] is listed. We treat the first two examples in some detail and summarize the results of the remaining cases in Appendix A.

\begin{tabular}{|c|r|r|r|r|r|}
\hline $\mathrm{X}$ & $X_{7}(1,1,1,2,2)$ & $X_{7}(1,1,1,1,3)$ & $X_{8}(1,1,1,2,3)$ & $X_{9}(1,1,2,2,3)$ & $X_{14}(1,1,2,3,7)$ \\
\hline$\left(h^{1,1}, h^{2,1}\right)$ & $(2,95)$ & $(2,122)$ & $(2,106)$ & $(2,86)$ & $(2,132)$ \\
$C(\Delta), C\left(\Delta^{*}\right)$ & $(9,6)$ & $(8,6)$ & $(8,6)$ & $(9,6)$ & $(7,6)$ \\
$\nu^{*(6)}$ & $(0,0,-1,-1)$ & $(0,0,0,-1)$ & $(0,0,0,-1)$ & $(0,-1,-1,-1)$ & $(0,0,-1,-2)$ \\
\hline$\hat{X}$ & $X_{21}(2,2,3,7,7)$ & $X_{14}(1,2,2,2,7)$ & $X_{8}(1,1,1,1,4)$ & $X_{12}(1,1,3,3,4)$ & $X_{28}(2,2,3,7,14)$ \\
\hline$\left(\hat{h}^{1,1}, \hat{h}^{2,1}\right)$ & $(11,50)$ & $(2,122)$ & $(1,149)$ & $(5,89)$ & $(9,83)$ \\
$H \quad: \quad \hat{h}^{(1)}$ & $\frac{1}{21}(2,1,18,7,14)$ & $\frac{1}{7}(1,0,6,0,0)$ & $\frac{1}{8}(7,0,0,1,0)$ & $\frac{1}{9}(1,8,0,0,0)$ & $\frac{1}{14}(1,13,0,0,0)$ \\
$: \quad \hat{h}^{(2)}$ & & $\frac{1}{7}(1,6,0,0,0)$ & $\frac{1}{8}(7,0,1,0,0)$ & $\frac{1}{4}(1,0,3,0,0)$ & $\frac{1}{2}(1,0,0,0,1)$ \\
\hline
\end{tabular}

Table 3.1: Non-Fermat Calabi-Yau hypersurfaces in $\mathbf{P}^{4}(\vec{w})$ with $h^{1,1}=2$. The table displays the Hodge numbers and the number of corners $\left(C(\Delta), C\left(\delta^{*}\right)\right)$ of the Newton polyhedron $\Delta$ and its dual $\Delta^{*}$ respectively. For the five corners of $\Delta^{*}$ besides $\nu^{*(6)}$, see (2.4) and the discussion below. Note that there are no non-toric states in $X$ because all non-interior points in $\Delta^{*}$ are corners or interior points of codimension one faces, which means that we can describe all complex- and Kähler-structure perturbations of $X$ algebraically. The mirror manifold is $X^{*}=\hat{X} / H$, were $\hat{X}$ is the transposed hypersurface with Hodge numbers $\hat{h}^{1,1}, \hat{h}^{2,1}$, which is transversal in these cases and hence appears in [25]. The vectors $h^{(k)}$ specify the generators of $H$, which act by $x_{i} \rightarrow x_{i} \exp 2 \pi i h_{i}^{(k)}$ on the homogeneous coordinates of $\hat{X}$.

The first model is defined by the zero locus $p=0$ of a quasihomogeneous polynomial of degree $d=7$ in the weighted projective space $\mathbf{P}^{4}(1,1,1,2,2)$. By Bertini's Theorem (see e.g. Remark III.10.9.2. in [48) transversality can fail for a generic member of $F$ only at the base locus, i.e. the locus where every $p$ vanishes identically. This base locus is the hyperplane $H=\left\{x \in \mathbf{P}^{4}(1,1,1,2,2) \mid x_{1}=x_{2}=x_{3}=0\right\}$. However the possible singular locus $S=\{x \in H \mid d p=0\}$, where transversality could fail, is empty for a generic member of $X$. A transversal member of $X$ is given for instance by

$$
X_{7}(1,1,1,2,2)=\left\{x \in \mathbf{P}^{4}(1,1,1,2,2) \mid p_{0}=x_{1}^{7}+x_{2}^{7}+x_{3}^{7}+x_{1} x_{4}^{3}+x_{2} x_{5}^{3}=0\right\} .
$$

Note that $p_{0}$ is not a sum of $A-D-E$ singularities, but since the transversal configuration involves only five terms the construction of ref. [32] will apply. All complex structure deformations are algebraic and can be described by 95 monomial perturbations of $p_{0}$ with 
elements of $\mathcal{R}=\mathbb{C}\left[x_{1}, \ldots, x_{5}\right] /\left\{\partial_{x_{1}} p_{0}, \ldots, \partial_{x_{5}} p_{0}\right\}$. The canonical resolution $\hat{X}$ of the hypersurface $X$ has two elements in $H^{2}(\hat{X})$; one corresponds to the divisor associated to the generating element of $\operatorname{Pic}(X)$ and a second one stems from the exceptional divisor, which is introduced by the resolution of the $Z_{2}$-singularity.

Returning to $\mathbf{P}^{4}(1,1,1,2,2)$, we see that $\Delta$ has nine corners whose components read

$$
\begin{array}{lcc}
\nu^{(1)}=(-1,-1,-1,2), & \nu^{(2)}=(-1,-1,-1,-1), & \nu^{(3)}=(0,-1,-1,2) \\
\nu^{(4)}=(6,-1,-1,-1), & \nu^{(5)}=(-1,0,-1,2), & \nu^{(6)}=(-1,6,-1,-1) \\
\nu^{(7)}=(-1,-1,2,-1), & \nu^{(8)}=(0,-1,2,-1), & \nu^{(9)}=(-1,0,2,-1)
\end{array}
$$

in a convenient basis for the sublattice $\Lambda \in \mathbb{Z}^{5}$ within the hyperplane: $e_{1}=(-1,1,0,0,0)$, $e_{2}=(-1,0,1,0,0), e_{3}=(-2,0,0,1,0)$ and $e_{4}=(-2,0,0,0,1)$. Beside the corners, $\Delta$ contains 1 lattice point in the interior, 20 lattice points on codimension 1 faces, 54 lattice points on codimension 2 and 36 lattice points on codimension 3 faces. One can pick 95 monomials corresponding to 4 of the corners, the internal point and the 90 points on codimension 2 and 3 faces as representatives of $\mathcal{R}$.

Since we know that the weights $w_{i}$ at hand admits a transverse polynomial the polyhedron $\Delta$ is reflexive 30. Its dual $\Delta^{*}$ has six corners, whose components in the basis of the dual lattice $\Lambda^{*}$ are given below

$$
\begin{array}{ccc}
\nu^{*(1)}=(-1,-1,-2,-2), & \nu^{*(2)}=(1,0,0,0), & \nu^{*(3)}=(0,1,0,0), \\
\nu^{*(4)}=(0,0,1,0), & \nu^{*(5)}=(0,0,0,1), & \nu^{*(6)}=(0,0,-1,-1) .
\end{array}
$$

Beside these corners, the point $\nu^{*(0)}=(0,0,0,0)$ is the only integral point in $\Delta^{*}$. In all cases where $w_{1}=1$ we can chose the lattice such that $\nu^{*(1)}=\left(-w_{2},-w_{3},-w_{4},-w_{5}\right)$ and $\nu^{*(i)}$ is as above for $i=2, \ldots, 5$. In all cases considered here, there is only the additional corner $\nu^{*(6)}$. It is always the case that $\nu^{*(0)}$ is an additional integral point of $\Delta^{*}$. It is in fact the only additional integral point except in the case of $\mathbf{P}^{4}(1,1,2,3,7)$. In that case $\Delta$ contains the point $(0,0,0,-1)$; but this plays essentially no role since it is the interior point of a codimension 1 face. Hence it is sufficient to only list $\nu^{*(6)}$ for the other cases, see Table 3.1.

Using (3.3) and (2.5) the Laurent polynomial of $\Delta^{*}$ is given by

$$
P=\sum_{i} a_{i} \phi_{i}=a_{0}+a_{1} \frac{1}{Y_{1} Y_{2} Y_{3}^{2} Y_{4}^{2}}+a_{2} Y_{1}+a_{3} Y_{2}+a_{4} Y_{3}+a_{5} Y_{4}+a_{6} \frac{1}{Y_{3} Y_{4}} .
$$

The étale map is given by

$$
Y_{1}=\frac{y_{2}^{6}}{y_{1} y_{3} y_{4}}, Y_{2}=\frac{y_{3}^{6}}{y_{1} y_{2} y_{4} y_{5}}, Y_{3}=\frac{y_{4}^{2}}{y_{1} y_{2} y_{3} y_{5}}, Y_{4}=\frac{y_{5}^{2}}{y_{1} y_{2} y_{3} y_{4}}
$$

which leads to a polynomial constraint

$$
\hat{p}=\sum_{i=0}^{6} a_{i} \phi_{i} \equiv a_{1} y_{1}^{7} y_{4}+a_{2} y_{2}^{7} y_{5}+a_{3} y_{3}^{7}+a_{4} y_{4}^{3}+a_{5} y_{5}^{3}+a_{0} y_{1} y_{2} y_{3} y_{4} y_{5}+a_{6}\left(y_{1} y_{2} y_{3}\right)^{3},
$$


which is quasihomogeneous of degree $\hat{d}=21$ with respect to the weights of $\mathbf{P}^{4}(2,2,3,7,7)$. In fact $\hat{p}_{0}=y_{1}^{7} y_{4}+y_{2}^{7} y_{5}+y_{3}^{7}+y_{4}^{3}+y_{5}^{3}$ is the transposed polynomial of $p_{0}$ in (3.1) and the symmetry, which is identified in (3.5) corresponds exactly to the symmetry group $H \sim$ $\left(\mathbf{Z}_{21}: 2,1,18,7,14\right)$, which has to be modded out from the configuration $X_{21}(2,2,3,7,7)$ to obtain the mirror [32] of the configuration $X_{7}(1,1,1,2,2)$. Note also that the family $X_{21}(2,2,3,7,7)$ admits 50 independent complex structure perturbations (which are all algebraic) but the terms of (3.6) are the only invariant terms under the $H$ symmetry group.

The boundary of $\Delta^{*}$ in $(3.3)$ consists of 9 three dimensional simplices and joining each of these simplices with the origin $\nu^{*(0)}$ we get a unique star subdivision of $\Delta^{*}$ into 9 four dimensional simplices. Application of the algorithm for constructing the generators of the Mori cone described in the previous section leads to

$$
l^{(1)}=(-3,0,0,0,1,1,1), \quad l^{(2)}=(-1,1,1,1,0,0,-2) .
$$

Note that the Mori cone of $\mathbf{P}_{\Delta^{*}}$ coincides with the Mori cone of $X$ in this example, as well as in all examples treated so far [22,46]; see however sections 3.2, 4 and Appendix A for models where this is not true. From the general discussion in section 2 this allows us to determine the relevant coordinates in the large complex structure limit, see (2.11), as $z_{1}=-\frac{a_{4} a_{5} a_{6}}{a_{0}^{3}}$ and $z_{2}=-\frac{a_{1} a_{2} a_{3}}{a_{0} a_{6}^{2}}$.

From the relations $\phi_{0}^{3}-\phi_{4} \phi_{5} \phi_{6}=0$ and $\phi_{6}^{2} \phi_{0}-\phi_{1} \phi_{2} \phi_{3}=0$, where the $\phi_{i}$ are defined by (3.4) or equivalently by (3.6), we get two third order differential operators satisfied by all periods $\partial_{a_{0}}^{3}-\partial_{a_{4}} \partial_{a_{5}} \partial_{a_{6}}=0$ and $\partial_{a_{6}}^{2} \partial_{a_{0}}-\partial_{a_{1}} \partial_{a_{2}} \partial_{a_{3}}=0$. This is readily transformed in the good variables (2.11)

$$
\begin{aligned}
& \mathcal{L}_{1}=\theta_{1}^{2}\left(2 \theta_{2}-\theta_{1}\right)+\left(3 \theta_{1}+\theta_{2}-2\right)\left(3 \theta_{1}+\theta_{2}-1\right)\left(3 \theta_{1}+\theta_{2}\right) z_{1} \\
& \mathcal{L}_{2}=\theta_{2}^{3}-\left(3 \theta_{1}+\theta_{2}\right)\left(2 \theta_{2}-\theta_{1}-2\right)\left(2 \theta_{2}-\theta_{1}-1\right) z_{2} .
\end{aligned}
$$

A short consideration of the indicial problem reveals that it has nine solutions. The six periods however are solutions of a system, which consists of a third and a second order differential operator. As in many examples in [46] the second order differential can be obtained in this case rather simply by factoring $7 \mathcal{L}_{2}-27 \mathcal{L}_{1}=\left(3 \theta_{1}+\theta_{2}\right) L_{2}$ with

$$
L_{2}=9 \theta_{1}^{2}-21 \theta_{1} \theta_{2}+7 \theta_{2}^{2}-27 z_{1} \prod_{i=1}^{2}\left(3 \theta_{1}+\theta_{2}+i\right)-7 z_{2} \prod_{i=0}^{1}\left(2 \theta_{2}-\theta_{1}+i\right) .
$$

As Picard-Fuchs system we may choose this operator and say $L_{1}=\mathcal{L}_{2}$.

Instead of using the factorization we may derive e.g. the second order differential operator from the vanishing of

$$
\begin{aligned}
\Xi= & a_{1} a_{2} \phi_{1} \phi_{2}-3 a_{1} a_{6} \phi_{1} \phi_{6}-3 a_{2} a_{6} \phi_{2} \phi_{6}-3 a_{0} a_{6} \phi_{0} \phi_{6}-27 z_{1} a_{0}^{2} \phi_{0}^{2}-7 z_{2} a_{6}^{2} \phi_{6}^{2}- \\
& \frac{9 a_{4} a_{6}}{a_{0}}\left(y_{1} y_{2} y_{3} y_{4}\right)^{2} \partial_{y_{5}} \hat{p}+3 a_{6}\left(\frac{a_{2}}{a_{0}} y_{1}^{2} y_{2}^{9} y_{3}^{2}+y_{4}\left(y_{1} y_{2} y_{3}\right)^{3}\right) \partial_{y_{4}} \hat{p}-\frac{a_{1} a_{2}}{a_{0}}\left(y_{1} y_{2}\right)^{6} \partial_{y_{3}} \hat{p}
\end{aligned}
$$


which implies $\int_{\gamma} \int_{\Gamma_{i}} \frac{\Xi \hat{\omega}}{\hat{p}^{3}}=0$. After the partial integration we get from the last three terms of $\Xi$ the contribution $\frac{3 a_{6}}{2} \int_{\gamma} \int_{\Gamma_{i}} \frac{\phi_{6} \omega}{\hat{p}^{2}}$. Replacing all $\phi_{i}$ by derivatives with respect to the $a_{i}$ and transforming to the $z_{i}$ variables yields (3.9) It is clear that (3.10) can be used analogously in (2.7), to obtain the second order differential relation. To be more precise $\Xi$ transforms into the variables $Y_{i}$ as

$$
\begin{aligned}
\Xi= & a_{1} a_{2} \phi_{1} \phi_{2}-3 a_{1} a_{6} \phi_{1} \phi_{6}-3 a_{2} a_{6} \phi_{2} \phi_{6}-3 a_{0} a_{6} \phi_{0} \phi_{6}-27 z_{1} a_{0}^{2} \phi_{0}^{2}-7 z_{2} a_{6}^{2} \phi_{6}^{2}- \\
& \frac{9 a_{4} a_{6}}{a_{0}} \frac{1}{Y_{4}} d_{1} P+3 a_{6}\left(\frac{a_{2}}{a_{0}} \frac{Y_{1}}{Y_{3} Y_{4}}+\frac{1}{Y_{3} Y_{4}}\right) d_{7} P-\frac{a_{1} a_{2}}{a_{0}} \frac{1}{Y_{2} Y_{3}^{2} Y_{4}^{2}} d_{6} P=0,
\end{aligned}
$$

where $d_{1}=\left(1-\Theta_{1}-\Theta_{2}-\Theta_{3}+2 \Theta_{4}\right), d_{7}=\left(1-\Theta_{1}-\Theta_{2}+2 \Theta_{3}-\Theta_{4}\right), d_{6}=\left(1-\Theta_{1}+\right.$ $6 \Theta_{2}-\Theta_{3}-\Theta_{4}$ ) and $\Theta_{i}=Y_{i} \frac{d}{d Y_{i}}$. Using the partial integration rule yields again (3.9).

From the Picard-Fuchs operators, we first determine the intersection numbers up to a normalization which we get from (2.15). Hence $K_{J_{1} J_{1} J_{1}}=14, K_{J_{1} J_{1} J_{2}}=7, K_{J_{1} J_{2} J_{2}}=3$ and $K_{J_{2} J_{2} J_{2}}=0$. Secondly, we derive the general discriminant locus

$$
\Delta=\left(1-27 z_{1}\right)^{3}-z_{2}\left(8-675 z_{1}+71442 z_{1}^{2}-16 z_{2}+1372 z_{1} z_{2}-453789 z_{1}^{2} z_{2}+823543 z_{1}^{2} z_{2}^{2}\right)
$$

and the Yukawa couplings in our normalization

$$
\begin{aligned}
K_{111} & =\frac{\left(14-112 z_{2}+324 z_{1}+729 z_{1}^{2}-2213 z_{1} z_{2}-1323 z_{1}^{2} z_{2}+224 z_{2}^{2}+4116 z_{1} z_{2}^{2}\right)}{z_{1}^{3} \Delta} \\
K_{112} & =\frac{\left(7-135 z_{1}-1458 z_{1}^{2}-56 z_{2}+1284 z_{1} z_{2}+3969 z_{1}^{2} z_{2}+112 z_{2}^{2}-2744 z_{1} z_{2}^{2}\right)}{z_{1}^{2} z_{2} \Delta} \\
K_{122} & =\frac{\left(3-162 z_{1}+2187 z_{1}^{2}-26 z_{2}+1629 z_{1} z_{2}-11907 z_{1}^{2} z_{2}+56 z_{2}^{2}-3773 z_{1} z_{2}^{2}\right)}{z_{1} z_{2}^{2} \Delta} \\
K_{222} & =\frac{z_{1}\left(-11+1161 z_{1}+35721 z_{1}^{2}+28 z_{2}-3087 z_{1} z_{2}\right)}{z_{2}^{3} \Delta} .
\end{aligned}
$$

Finally, the number of rational curves is obtained by an expansion of (3.13) around $z_{i}=0$; the result is recorded in Table 3.2 .

To obtain the invariants for the elliptic curves we first note that $\int_{X} c_{2} J_{1}=68$ and $\int_{X} c_{2} J_{2}=36$. Using the ansatz (2.18) and the asymptotic relation (2.20) one obtains $s_{1}=-20 / 3, s_{2}=-4$. The invariants of the elliptic curves still contain $r_{0}$, e.g. $n_{0,1}^{e}=$ $-(1 / 3)\left(2+12 r_{0}\right)$. We will see in section 4.1 that this invariant vanishes, which fixes $r_{0}=-(1 / 6)$. This allows us to obtain the other invariants of the elliptic curves, see Table 3.2. In fact, we will see later that $n_{0,1}^{e}=0$ for all of our two parameter models, which will allow us to determine $r_{0}$ in each case. We always obtain $r_{0}=-1 / 6$, which supports the conjecture [49], [46] that the exponent is universally -(1/6) for the component of the discriminant parameterizing nodal hypersurfaces. An intriguing possible explanation of 
this phenomenon based on consideration of black hole states of the type II string has been given in [50]. These instanton predictions will be discussed in section four. The successful check provides a very detailed verification that the configuration $X_{21}(2,2,3,7,7)$ modded out by $H \simeq\left(\mathbb{Z}_{21}: 2,1,18,7,14\right)$ is in fact the correct mirror configuration.

\begin{tabular}{|l|rrrrrrr|}
\hline$n_{i, j}$ & $j=0$ & $j=1$ & $j=2$ & $j=3$ & $j=4$ & $j=5$ & $j=6$ \\
\hline$n_{0, j}^{r}$ & 0 & -2 & 0 & $\forall j>1$ & & & \\
$n_{0, j}^{e}$ & 0 & $\forall j$ & & & & & \\
\hline$n_{1, j}^{r}$ & 177 & 178 & 3 & 5 & 7 & 9 & 11 \\
$n_{1, j}^{e}$ & 0 & $\forall j$ & & & & & \\
\hline$n_{2, j}^{r}$ & 177 & 20291 & -177 & -708 & -1068 & -1448 & 1880 \\
$n_{2, j}^{e}$ & 0 & 0 & 0 & 0 & 0 & 9 & 68 \\
\hline$n_{3, j}^{r}$ & 186 & 317172 & 332040 & 44790 & 75225 & 110271 & 157734 \\
$n_{3, j}^{e}$ & 3 & 4 & 181 & 534 & 885 & -177 & -11161 \\
\hline$n_{4, j}^{r}$ & 177 & 2998628 & 73458379 & 794368 & -4468169 & -7157586 & -11253268 \\
$n_{4, j}^{e}$ & 0 & -356 & 316802 & -60844 & -121684 & -81636 & 857218 \\
\hline$n_{5, j}^{r}$ & 177 & 21195310 & 3048964748 & 3122149716 & 243105088 & 396368217 & 676476353 \\
$n_{5, j}^{e}$ & 0 & -40582 & 21251999 & 26695536 & 16380749 & 23269402 & -21423697 \\
\hline
\end{tabular}

Table 3.2 The invariants of rational and elliptic curves of degree $(i, j), n_{i, j}^{r}$ and $n_{i, j}^{e}$ respectively, for $X_{7}(1,1,1,2,2)$. (The computation was done using the Mathematica code, INSTANTON [46].)

The mirror configuration for the second example $X_{7}(1,1,1,1,3)$ is obtained by the resolved quotient of the family $X_{14}(1,2,2,2,7)$, a manifold with $h^{2,1}=2$ and $h^{1,1}=$ 122. These are in fact also the Hodge numbers of the mirror of the Fermat configuration $X_{14}(1,2,2,2,7)$ itself, which suggests, that $X_{7}(1,1,1,1,3)$ and $X_{14}(1,2,2,2,7)$ (as well as its mirror pair) are isomorphic. This has been checked to be the case in [30]. Let us look into this in more detail and check first the homotopy type of the mirror pair. We get the following generators of the Mori cone

$$
l^{(1)}=(-2,0,0,0,0,1,1), \quad l^{(2)}=(-1,1,1,1,1,0,-3)
$$

Applying similar methods as described above we derive a third and a second order PicardFuchs equation

$$
\begin{aligned}
& \mathcal{L}_{1}=\theta_{1}\left(\theta_{1}-3 \theta_{2}\right)-\left(2 \theta_{1}+\theta_{2}\right)\left(2 \theta_{1}+\theta_{2}-1\right) z_{1} \\
& \mathcal{L}_{2}=\theta_{2}^{2}\left(7 \theta_{2}-2 \theta_{1}\right)+4 \theta_{2}^{2}\left(2 \theta_{1}+\theta_{2}-1\right) z_{1}-7 \prod_{i=1}^{3}\left(2 \theta_{2}-\theta_{1}-i\right),
\end{aligned}
$$


from which the ratio of the topological couplings can be read off. Fixing, according to [47], the intersection $K_{J_{1} J_{1} J_{1}}=63$, we get $K_{J_{1} J_{1} J_{2}}=21, K_{J_{1} J_{2} J_{2}}=7$ and $K_{J_{2} J_{2} J_{2}}=2$. In terms of the complex structure parameters $z_{1}, z_{2}$ the Yukawa couplings are given as follows

$$
\begin{aligned}
& K_{111}=\frac{\left(63+217 z_{1}+168 z_{1}^{2}+16 z_{1}^{3}+63 z_{2}\left(3+7 z_{1}\right)\left(9+7 z_{1}\right)\right)}{z_{1}^{3} \Delta} \\
& K_{112}=\frac{z_{2}\left(21-42 z_{1}-160 z_{1}^{2}-32 z_{1}^{3}+7 z_{2}\left(81-189 z_{1}-539 z_{1}^{2}\right)\right)}{z_{1}^{2} \Delta} \\
& K_{122}=\frac{z_{2}^{2}\left(7-52 z_{1}+80 z_{1}^{2}+64 z_{1}^{3}+7 z_{2}\left(9-28 z_{1}\right)\left(3-14 z_{1}\right)\right)}{z_{1} \Delta} \\
& K_{222}=\frac{\left(2-24 z_{1}+96 z_{1}^{2}-128 z_{1}^{3}+7 z_{2}\left(9-119 z_{1}+588 z_{1}^{2}\right)\right)}{z_{2}^{3} \Delta}
\end{aligned}
$$

where the general discriminant $\Delta$ is

$$
\Delta=\left(1-4 z_{1}\right)^{4}+27 z_{2}-411 z_{1} z_{2}+2744 z_{1}^{2} z_{2}-38416 z_{1}^{3} z_{2}-823543 z_{1}^{3} z_{2}^{2}
$$

Using the formulas from [46] one obtains $\int c_{2} J_{1}=126, \int c_{2} J_{2}=44$. Comparing with [22], [46] we see that these topological numbers indeed coincide with the ones of the mirror of the Fermat model $X_{14}(1,2,2,2,7)$ after exchanging the rôle of the exceptional divisor and the one from the ambient space. The Picard-Fuchs equations and the expression for the Yukawa couplings however do not coincide after exchanging $z_{1}$ and $z_{2}$. The difference is in fact in the choice of the normalization of the holomorphic three-form and one of the complex structure coordinates. The other complex structure coordinate can be identified. If we denote by $\tilde{z}_{i}$ the coordinate of the $X_{14}(1,2,2,2,7)$ model associated to $l^{(1)}=(-7,0,1,1,1,-3,7)$ (in the notation of [22]), we have the following simple relation between the mirror maps in both models: $\tilde{z}_{1}\left(q_{1}, q_{2}\right)=z_{2}\left(q_{2}, q_{1}\right)$. The resulting topological invariants, modulo the interchange of the divisors, are exactly the ones calculated in [22], [46] for $X_{14}(1,2,2,2,7)$. We will further discuss the relation between the models in section five. The first few are listed below in Table 3.3. Note that also in this case, as well as in all other cases we consider here, the exponent of the holomorphic ambiguity at the general discriminant turns out to be $r_{0}=-1 / 6$. 


\begin{tabular}{|l|rrrrrrr|}
\hline$n_{i, j}$ & $j=0$ & $j=1$ & $j=2$ & $j=3$ & $j=4$ & $j=5$ & $j=6$ \\
\hline$n_{j, 0}^{r}$ & 0 & 28 & 0 & $\forall j>1$ & & & \\
$n_{j, 0}^{e}$ & 0 & $\forall j$ & & & & & \\
\hline$n_{j, 1}^{r}$ & 3 & -56 & 378 & 14427 & 14427 & 378 & -56 \\
$n_{j, 1}^{e}$ & 0 & $\forall j$ & & & & & \\
\hline$n_{j, 2}^{r}$ & -6 & 140 & -1512 & 9828 & -69804 & 500724 & 29683962 \\
$n_{j, 2}^{e}$ & 0 & 0 & 0 & 0 & 0 & 378 & 6496 \\
\hline$n_{j, 3}^{r}$ & 27 & -896 & 13426 & -122472 & 837900 & -5083092 & 27877878 \\
$n_{j, 3}^{e}$ & -10 & 252 & -3024 & 22932 & -122850 & 489888 & -1474200 \\
\hline
\end{tabular}

Table 2.3 The invariants of rational and elliptic curves of degree $(i, j), n_{i, j}^{r}$ and $n_{i, j}^{e}$ respectively, for $X_{7}(1,1,1,1,3)$.

\subsection{An Exotic Example}

As the simplest example, in terms of the number of Kähler deformations, for which the naive application of [32] fails we now consider a hypersurface of degree 13 in $\mathbf{P}^{4}(1,2,3,3,4)$ that has Euler number -114. The second Betti number is 5 . In this case we do not expect to find a subsector of the algebraic deformations from a LG-potential to be related to the complexified Kähler structure deformation of $X_{13}(1,2,3,3,4)$. Instead we should learn everything from the dual polyhedron $\Delta^{*}$, whose corners are the following points

$$
\begin{aligned}
& \nu^{*(1)}=(-2,-3,-3,-4), \quad \nu^{*(2)}=\left(\begin{array}{llll}
1, & 0, & 0, & 0
\end{array}\right), \quad \nu^{*(3)}=\left(\begin{array}{llll}
0, & 1, & 0, & 0
\end{array}\right),
\end{aligned}
$$

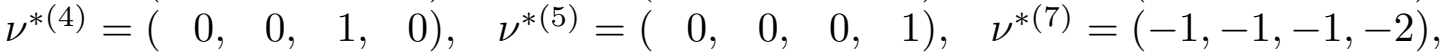

$$
\begin{aligned}
& \nu^{*(8)}=(-1,-2,-2,-2), \quad \nu^{*(9)}=(-1,-2,-2,-3) \text {, }
\end{aligned}
$$

and which contains in addition the point $\nu^{*(6)}=(0,-1,-1,-1)$ on the edge $\left(\nu^{*(2)}-\right.$ $\left.\nu^{*(8)}\right)$ apart from the interior point $\nu^{*(0)}=(0,0,0,0)$. In order to find the generators of the Mori cone, we have to specify a particular triangulation; more precisely a star subdivision of $\Delta^{*}$ from the point $\nu^{*(0)}$. This triangulation is not unique, in contrast to the previous examples. First note that $\Delta^{*}$, which has volume 22 is bounded by thirteen three-dimensional hyperplanes, on which the natural bilinear form $\left\langle\nu^{(i)},.\right\rangle i=1, \ldots, 13$ takes the value -1 for $\nu^{(i)}$ the corners of $\Delta$

$$
\begin{aligned}
& \nu^{(1)}=(-1,-1,-1,2), \quad \nu^{(2)}=(-1,-1,-1,-1), \quad \nu^{(3)}=(5,-1,-1,-1), \\
& \nu^{(4)}=(0, \quad 0,-1,1), \quad \nu^{(5)}=(4,0,-1,-1), \quad \nu^{(6)}=(-1,2,-1,0) \text {, } \\
& \nu^{(7)}=(1,2,-1,-1), \quad \nu^{(8)}=(-1,3,-1,-1) \quad \nu^{(9)}=(0,-1,0,1) \text {, } \\
& \nu^{(10)}=(4,-1, \quad 0,-1), \quad \nu^{(11)}=(-1,-1, \quad 2,0), \quad \nu^{(12)}=(1,-1,2,-1) \text {, } \\
& \nu^{(13)}=(-1,-1, \quad 3,-1) \text {. }
\end{aligned}
$$


There exists six different subdivisions which admit a Kähler resolution 9. One of them is given by 10

$$
\begin{array}{cccc}
s_{1}=(3,4,7,9), & s_{2}=(3,4,5,7), & s_{3}=(2,3,4,5), & s_{4}=(2,3,5,6), \\
s_{5}=(2,4,5,6), & s_{6}=(2,3,4,9), & s_{7}=(4,5,6,8), & s_{8}=(3,5,6,8), \\
s_{9}=(3,5,1,7), & s_{10}=(1,4,5,7), & s_{11}=(1,4,7,9), & s_{12}=(1,3,7,9), \\
s_{13}=(1,3,5,8), & s_{14}=(1,4,5,8), & s_{15}=(1,2,4,6), & s_{16}=(1,2,3,6), \\
s_{17}=(1,3,6,8), & s_{18}=(1,4,6,8), & s_{19}=(1,2,4,9), & s_{20}=(1,2,3,9) .
\end{array}
$$

We now apply the algorithm in section 2 ; step iv) is straightforward but tedious. The generators of the Mori cone are 11

$$
\begin{aligned}
& l_{1}=(-1, \quad 1, \quad 0, \quad 0, \quad 0, \quad 1, \quad 1, \quad 0,-2, \quad 0) \\
& l_{2}=(0, \quad 0, \quad 1,-1,-1, \quad 0, \quad 0,3, \quad 0,-2) \\
& l_{3}=(-1, \quad 1, \quad 0, \quad 1, \quad 1, \quad 0, \quad 0,-2, \quad 0, \quad 0)
\end{aligned}
$$

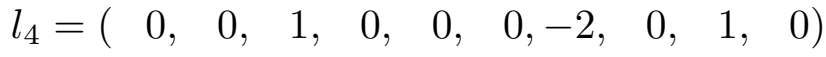

$$
\begin{aligned}
& l_{5}=(0,-1,-1, \quad 0, \quad 0, \quad 0, \quad 1, \quad 0, \quad 0, \quad 1)
\end{aligned}
$$

From the dual of the Mori cone (which is the Kähler cone of the toric variety in which $X_{13}(1,2,3,3,4)$ is a hypersurface) we get the classical intersection numbers, see Table B.1. As a check, the identical intersection numbers have been calculated using [53].

To each of the above generators we associate a coordinate in the usual way, i.e.

$$
z_{1}=-\frac{a_{1} a_{5} a_{6}}{a_{8}^{2} a_{0}}, \quad z_{2}=\frac{a_{2} a_{7}^{3}}{a_{3} a_{4} a_{9}^{2}}, \quad z_{3}=-\frac{a_{1} a_{3} a_{4}}{a_{0} a_{7}^{2}}, \quad z_{4}=\frac{a_{2} a_{8}}{a_{6}^{2}}, \quad z_{5}=\frac{a_{6} a_{9}}{a_{1} a_{2}} .
$$

As in the previous example, we use the residue expression for the period integral to derive the differential equation,

$$
\Pi_{i}\left(a_{0}, \ldots, a_{p}\right)=\int_{\gamma} \int_{\Gamma_{j}} \frac{\omega}{P}, \quad j=1, \ldots, 2\left(h^{2,1}+1\right),
$$

9 In this respect the model is similar to the much celebrated example of topology change, studied in [41]. We will however refrain from considering this aspect since our motivation is different; although an interesting problem, the outcome would not imply anything new in terms of the phase structure [51.

10 This subdivision and the other Kähler subdivisions were found from among the 305 subdivisions (phases) produced by the computer program PUNTOS written by J. De Loera [52].

11 There is an interesting point here. The Mori cone calculated here is the Mori cone of the toric variety determined by our triangulation of $\Delta^{*}$. This cone differs from the Mori cone of the Calabi-Yau hypersurface. To our knowledge, this is the first known example of this phenomenon; see also Appendix A.3. We return to this point in section 4.3. 
where $P$ is the Laurent polynomial, $P=\sum_{i} a_{i} \phi_{i} \equiv \sum_{i} a_{i} \prod_{j} Y_{j}^{\nu_{j}^{*(i)}}$. Contrary to the examples earlier in this section we do not have an expression for which the mirror is defined as a quotient of a model defined by a transverse hypersurface in a weighted projective space. However, we can still write down the differential operators based on the relations between the $\phi_{i}$ or equivalently between the $l_{i}$, the generators of the Mori cone given in (3.18). At order two and three we get 6 and 13 differential operators in this fashion. Since there are five moduli, we would need 10 differential equations of order two - there are only five nontrivial elements of degree 2 , but $5 \cdot 6 / 2=15$ "monomials" $\phi_{i} \phi_{j}$, with the indices running over the five moduli, which give ten relations. In most cases (see e.g. [22,46]) this problem can be solved by factorization, as was the case in the previous example. However, one can check (e.g. by using the code INSTANTON) that this is not the case here. As discussed in 22 one can circumvent this obstacle by explicitly using the ideal based on the defining polynomial by which the mirror is defined. Since we lack a monomial representation of the complex structure deformations in terms of a defining equation 12 , we will make use of the construction of a ring structure due to Batyrev and Cox [37] as discussed in section 2. One then gets the following polynomial

$$
\begin{aligned}
W= & a_{1} x_{1} x_{2}^{13} x_{3} x_{8} x_{13}+a_{2} x_{3}^{6} x_{4} x_{5}^{5} x_{7}^{2} x_{9} x_{10}^{5} x_{12}^{2}+a_{3} x_{4} x_{5} x_{6}^{3} x_{7}^{3} x_{8}^{4}+a_{4} x_{9} x_{10} x_{11}^{3} x_{12}^{3} x_{13}^{4}+ \\
& a_{5} x_{1}^{3} x_{4}^{2} x_{6} x_{9}^{2} x_{11}+a_{6} x_{1} x_{2}^{4} x_{3}^{4} x_{4} x_{5}^{3} x_{7} x_{9} x_{10}^{3} x_{12}+a_{7} x_{2}^{6} x_{6} x_{7} x_{8}^{2} x_{11} x_{12} x_{13}^{2}+ \\
& a_{8} x_{1}^{2} x_{2}^{8} x_{3}^{2} x_{4} x_{5} x_{9} x_{10}+a_{9} x_{2}^{9} x_{3}^{3} x_{5}^{2} x_{7} x_{8} x_{10}^{2} x_{12} x_{13}+a_{10} x_{1} \ldots x_{13}
\end{aligned}
$$

The idea is now to use the traditional reduction scheme à la Dwork-GriffithsKatz 38,39,40]. It is rather straightforward to go through the second order monomials, $\phi_{i} \phi_{j}$ and to show that the only non-trivial relation is 13

$$
a_{1} \phi_{9}^{2}+a_{0} \phi_{1} \phi_{7}+3 a_{5} \phi_{0} \phi_{6}+a_{6} \phi_{1} \phi_{9}+2 a_{8} \phi_{1} \phi_{5}=0 \text {. }
$$

Note that unlike the generic case of the reduction method there is no lower order piece from the partial integration.

This is still three short of the ten operators which we need, i.e. at the current level the ring is not adequate for describing the moduli space of interest. Rather than insisting on using all of the thirteen $x_{i}$, let us now restrict to $x_{1}, x_{2}, x_{5}, x_{8}, x_{11}$, i.e. formally set

12 Alternatively, we could have used the Laurent polynomial and the relations from the ideal as explained in section 2. However, we find it easier to use method outlined below.

13 Strictly speaking there is one further relation involving a second order monomial which cannot be written as $\phi_{i} \phi_{j}$, a phenomenon which was treated in [22]. However, as it turns out the third order relations (differential operators) which are obtained in this way are encompassed by the second order operators found below. 
the remaining $x_{i}=1$. Renaming these $y_{i}, i=1, \ldots, 5$ the Batyrev-Cox potential is then reduced to

$$
\begin{aligned}
\tilde{W}= & a_{1} y_{1} y_{2}^{13} y_{4}+a_{2} y_{3}^{5}+a_{3} y_{3} y_{4}^{4}+a_{4} y_{5}^{3}+a_{5} y_{1}^{3} y_{5}+ \\
& a_{0} y_{1} y_{2} y_{3} y_{4} y_{5}+a_{6} y_{1} y_{2}^{4} y_{3}^{3}+a_{7} y_{2}^{6} y_{4}^{2} y_{5}+a_{8} y_{1}^{2} y_{2}^{8} y_{3}+a_{9} y_{2}^{9} y_{3}^{2} y_{4} .
\end{aligned}
$$

Note that the first five terms can be thought of as obtained from a transposition of a degenerate potential for the original model, degenerate because we need to add extra terms in order to make it transversal; this was indeed the reason why the above $x_{i}$ were chosen. Since the $x_{i}$ correspond to points in $\Delta$, choosing the five $x_{i}$ corresponds to refraining from resolving the singularities of $X$, the existence of which the remaining $x_{i}$ are based on.

Finally, let us then consider the following three monomials

$$
\phi_{6} \phi_{8}, \quad \phi_{7}^{2}, \quad \phi_{0} \phi_{7}
$$

which are the among the fifteen $\phi_{i} \phi_{j}, i, j=0,6, \ldots, 9$ which do not appear in any of the previous relations at second order among the $\phi_{i} \phi_{j}$. By vigorous application of the ideal, $\partial \tilde{W}$, it is possible in all three cases to arrive at non-trivial relations of second order involving only monomials of the form $\phi_{i} \phi_{j}$. This completes the story; together with the operators obtained from the Mori cone, (3.18), and (3.21) it can be shown that the triple intersection numbers on the original toric variety $\mathbf{P}^{4}(1,2,3,3,4)$ are reproduced, see Table B.1. Note that they differ from those we would compute from the Kähler cone of the manifold $X_{14}(1,2,3,3,4)$, see the discussion in section 4.3. In Table B.2 we record the lowest order instanton corrections, some of which are verified in the following section.

\section{Verifications.}

In this section we geometrically explain some of the instanton numbers computed in the earlier sections.

\section{1. $\mathbf{P}^{4}(1,1,1,2,2)$}

The first step is to desingularize $\mathbf{P}^{4}(1,1,1,2,2)$. This has already been accomplished implicitly in (3.3) by the presence of the vector $\nu^{*(6)}$, since the other 5 vectors in (3.3) determine the toric fan for $\mathbf{P}^{4}(1,1,1,2,2)$. However, we prefer to use an explicit calculation.

$\mathbf{P}^{4}(1,1,1,2,2)$ is singular along the $\mathbf{P}^{1}$ defined by $x_{1}=x_{2}=x_{3}=0$. We desingularize by using an auxiliary $\mathbf{P}^{2}$ with coordinates $\left(y_{1}, y_{2}, y_{3}\right)$ and define

$$
\tilde{\mathbf{P}^{4}} \subset \mathbf{P}^{4}(1,1,1,2,2) \times \mathbf{P}^{2}
$$


by the equations

$$
x_{i} y_{j}=x_{j} y_{i}, \quad i, j=1,2,3 .
$$

The exceptional divisor is just $\mathbf{P}^{1} \times \mathbf{P}^{2}$, where the two projective spaces have coordinates $\left(x_{4}, x_{5}\right)$ and $\left(y_{1}, y_{2}, y_{3}\right)$ respectively. The proper transform of a general degree 7 hypersurface $X$ is seen to intersect the exceptional divisor in a surface defined by a polynomial $f\left(x_{4}, x_{5}, y_{1}, y_{2}, y_{3}\right)$ which is cubic in the $x$ 's and linear in the $y$ 's. This can be seen by direct calculation, the essential point being the presence of monomials cubic in $x_{4}, x_{5}$ and linear in $x_{1}, x_{2}, x_{3}$ in an equation for $X$. The fibers of the projection of this surface to $\mathbf{P}^{1}$ are lines; thus the desingularized Calabi-Yau manifold $\tilde{X}$ contains a ruled surface with $\mathbf{P}^{1}$ as a base.

This fact was noticed and pointed out to us by D.R. Morrison. Other examples of Calabi-Yau manifolds containing ruled surfaces parameterized by curves of higher genus have been studied in [46] and in [21]. The presence of a ruled surface with $\mathbf{P}^{1}$ as base yields different and interesting geometry; this has in part motivated us to calculate the instanton numbers for this example.

To understand this ruled surface, we first think of $\mathbf{P}^{1} \times \mathbf{P}^{2}$ as the projective bundle $\mathbf{P}\left(\mathcal{O}^{3}\right)$ on $\mathbf{P}^{1}$ (see [54 for notation). Writing $f=f_{1}(x) y_{1}+f_{2}(x) y_{2}+f_{3}(x) y_{3}$, we see that $f$ is determined by the three $f_{i}$; these can be identified with a map $\mathcal{O}(-3) \rightarrow \mathcal{O}^{3}$; the cokernel of a generic such map is $\mathcal{O}(1) \oplus \mathcal{O}(2)$. So the exceptional divisor of $X$ is just $\mathbf{P}(\mathcal{O}(1) \oplus \mathcal{O}(2))$. Rational ruled surfaces such as this are very well understood [54]. We quickly review the relevant points.

Let $B$ denote the rank 2 bundle $\mathcal{O}(a) \oplus \mathcal{O}(b)$ on $\mathbf{P}^{1}$ for some integers $a \leq b$. The abstract rational ruled surface $\mathbf{P}(B)$ is isomorphic to the Hirzebruch surface $F_{b-a}$; this surface is characterized by the minimum value of $C^{2}$ for $C$ a section of the ruled surface, the minimum being $a-b$. The section achieving this minimum is even unique if $a<b$.

$H^{2}(\mathbf{P}(B))$ is generated by two classes - a hyperplane class $H$ and the fiber $f$. The cohomology $H^{0}(m H+n f)$ is calculated for $m \geq 0$ as $H^{0}\left(\mathbf{P}^{1}, \operatorname{Sym}^{m}(B) \otimes \mathcal{O}(n)\right)$. The space of curves in the class $m H+n f$ is parameterized by the projectivization of this vector space. We also note the intersection numbers $H^{2}=a+b, H \cdot f=1, f^{2}=0$. In our case, this gives

$$
H^{2}=3, H \cdot f=1, f^{2}=0 .
$$

We also note that the curve of minimum self intersection is in the class $H-2 f$.

In particular, if any families of rational curves are of this type, and are parameterized by $\mathbf{P}^{r}$, then its contribution to the Gromov-Witten invariant for this type of curve is $(-1)^{r}(r+1)$ by [21]. Later, we will also need that if a family of rational curves is parameterized by a smooth curve of genus g, then its contribution to the Gromov-Witten invariant is $2 g-2$. 
The classes $J_{1}$ and $J_{2}$ are interpreted as the classes given by the zeros of quadratic and linear polynomials, respectively. Since all linear polynomials vanish along $x_{1}=x_{2}=$ $x_{3}=0$, and therefore along the exceptional divisor $E$ after the blowup, we conclude that $J_{1}=2 J_{2}+E$.

To compute $n_{i, j}^{r}$, we note that if $i<2 j$ and if $C \cdot J_{1}=i, C \cdot J_{2}=j$, then $C \cdot E=$ $i-2 j<0$, which implies that a component of $C$ must be contained in $E$. So in this case we are often reduced to understanding curves in $E$. Next, we need to observe that $\left.J_{1}\right|_{E} \simeq f$ and $\left.J_{2}\right|_{E} \simeq H$.

In the case of $n_{0, j}^{r}$ for $j \geq 1$, we see inductively that all of these curves lie in $E$. For these curves $C$, we have that (thought of as a curve in the ruled surface $E$ ) $C \cdot f=0$ and $C \cdot H=j$. The first equation and (4.2) say that $C$ must be a fiber; in that case, necessarily $C \cdot H=1$, so $j=1$. If $j=1$, then we have

$$
H^{0}(\mathbf{P}(B), f) \simeq H^{0}\left(\mathbf{P}^{1}, \mathcal{O}(1)\right) \simeq \mathbb{C}^{2}
$$

so the curves are parameterized by $\mathbf{P}^{1}$ and $n_{0,1}^{r}=-2$. We have also shown that $n_{0, j}^{r}=0$ for $j \geq 2$.

For $n_{1, j}^{r}$ with $j \geq 2$, these curves $C$ are again contained in $E$ (at this point, it is clear that a component is contained in $E$; but we will check later that all such curves are entirely contained in $E$ ).

We have $C \cdot f=1$ and $C \cdot H=j$, which implies that $C$ is of type $H+(j-3) f$. In addition, $C \cdot f=1$ implies that $C$ is a section or the union of a section and fibers, hence is rational. Finally, $H^{0}(\mathbf{P}(B), H+(j-3) f)$ is isomorphic to

$$
H^{0}\left(\mathbf{P}^{1}, B \otimes \mathcal{O}(j-3)\right)=H^{0}\left(\mathbf{P}^{1}, \mathcal{O}(j-2) \oplus \mathcal{O}(j-1)\right),
$$

which has dimension $2 j-1$. So the curves are parameterized by a projective space of dimension $2 j-2$. This gives $n_{1, j}=2 j-1$ for $j \geq 2$.

Note in passing that we have also shown that $n_{j, k}^{e}=0$ for $j=0,1$, since only rational curves arise as sections or fibers. In fact, for all of our two parameter models we note that for exactly the same reason as given above, a curve $C$ of type $(0,1)$ necessarily lies in the exceptional divisor $E$. In each case, $E$ is an explicitly given rational surface, and by consideration of the possible curves on $E$, the condition $C \cdot J_{2}=1$ is either not possible or forces $C$ to be rational. Either way, we get $n_{0,1}^{e}=0$, justifying the procedure given in section 3.1 for determining $r_{0}$.

Consider $n_{2,5}^{e}$. These curves are contained in $E$ by the now-familiar argument. Since $C \cdot f=2$ and $C \cdot H=5$, we get that $C$ is in the class $2 H-f$. This is a family of elliptic curves, and is parameterized by a $\mathbf{P}^{8}$. This gives $n_{2,5}^{e}=9$. The general curve in the class $2 H+b f$ is elliptic only for $b=-1$. This leads quickly to the conclusion that $n_{2, j}^{e}=0$ for $j \neq 5$. 
We can make further verifications (and finish an earlier argument) by using $J_{2}$. The three linear forms which are the sections of $J_{2}$ give a map to $\mathbf{P}^{2}$. Actually, we study this by means of the map $g: \tilde{\mathbf{P}^{4}} \rightarrow \mathbf{P}^{2}$ given by projection onto $\left(y_{1}, y_{2}, y_{3}\right)$; the map on the Calabi-Yau hypersurface $\tilde{X}$ is given by restriction. The fibers of $g$ are isomorphic to $\mathbf{P}^{2}$; in fact $\tilde{\mathbf{P}}^{4}$ can be constructed as the projective bundle $\mathbf{P}\left(\mathcal{O}^{2} \oplus \mathcal{O}(2)\right)$ on $\mathbf{P}^{2}$. The $\mathcal{O}^{2}$ corresponds to the coordinates $x_{4}, x_{5}$; the $\mathcal{O}(2)$ arises because $y_{1}, y_{2}, y_{3}$ can be rescaled in $\tilde{\mathbf{P}}^{4}$ independent of $x_{4}, x_{5}$. Like all projective bundles, this one comes with a tautological rank 1 quotient, the bundle of linear forms in the fibers. In this case, it turns out to be $J_{1}$. That is, there is a map $g^{*}\left(\mathcal{O}^{2} \oplus \mathcal{O}(2)\right) \rightarrow J_{1}$. The hypersurface $\tilde{X}$ is in the class $3 J_{1}+J_{2}$. This says that fibers of $g$, restricted to the hypersurface of interest, are plane cubic curves. The fibers are readily seen to be of type $(3,0)$.

We are now equipped to compute $n_{1,0}^{r}$. If $C \cdot J_{2}=0$, then $C$ is contained in a fiber of $g$. The condition $C \cdot J_{1}=1$ says that $C$ is a line. Hence we must enumerate points of $\mathbf{P}^{2}$ with the property that the cubic fiber factors into a line and a conic. In particular, there is a 1-1 correspondence between lines and conics; hence $n_{1,0}^{r}=n_{2,0}^{r}$.

The technique is standard [55]. We form the Grassmann bundle $G=\operatorname{Gr}^{2}\left(\mathcal{O}^{2} \oplus \mathcal{O}(2)\right)$ of lines in the fibers of $g$. Note that $\operatorname{dim}(G)=4$. There is a projection map $\phi: G \rightarrow \mathbf{P}^{2}$. On $G$, there is a canonical rank 2 bundle $Q$, the bundle of linear forms on these lines. The equation of $\tilde{X}$ induces a section of the rank 4 bundle $\operatorname{Sym}^{3}(Q) \otimes \phi^{*} \mathcal{O}(1)$. The line is contained in $\tilde{X}$ if and only if this section vanishes at the corresponding point of $G$. So we must calculate $c_{4}\left(\operatorname{Sym}^{3}(Q) \otimes \phi^{*} \mathcal{O}(1)\right)$. This is immediately calculated to be 177 using Schubert [53].

Turning to $n_{1,1}^{r}$, we note that $C \cdot J_{1}=1$ and $C \cdot J_{2}=1$ implies that a component of $C$ is contained in $E$. There are two cases: either $C \subset E$, or $C$ is a union of a curve of type $(1,0)$ and a curve of type $(0,1)$ (we have already seen that the latter curve is a fiber of $E$ ).

In the former case, $C \cdot f=1$ and $C \cdot H=1$ implies that $C$ is in the class $H-2 f$. Since $H^{0}(\mathbf{P}(B), H-2 f)$ is 1 dimensional, there is only one curve of this type, contributing 1 to $n_{0,1}^{r}$. Alternatively, note that $(H-2 f)^{2}=-1$, the minimum value; hence $C$ is unique.

If on the other hand $C=C^{\prime} \cup C^{\prime \prime}$ with $C^{\prime}$ of type $(1,0)$, then $C^{\prime} \cdot E=C^{\prime} \cdot\left(J_{1}-2 J_{2}\right)=1$. For $C$ to be connected, $C^{\prime \prime}$ must be the unique fiber $f$ containing the unique point of intersection on $C^{\prime}$ and $E$. So each curve of type $(1,0)$ yields a degenerate instanton (see appendix to [8]) of type $(1,1)$.

Putting these two cases together, we see that $n_{1,1}^{r}=n_{1,0}^{r}+1=178$.

Returning to the final details of $n_{1, j}^{r}$ for $j \geq 2$, we see that if $C$ were not contained in $E$, then it would have to be a union

$$
C=C^{\prime} \cup C_{1} \cup \ldots \cup C_{j}
$$

where $C^{\prime}$ has type $(1,0)$ and the $C_{i}$ have type $(0,1)$. But $C^{\prime}$ meets $E$ in just one point, so there is no way to add on $j$ fibers and obtain a connected curve. So this case does not occur, as asserted earlier. 
Next, $n_{3,0}^{e}$ is easy; these are the elliptic cubic curves in the fibers of $g$. But the general fibers of $g$ are elliptic cubic curves. So these curves are parameterized by $\mathbf{P}^{2}$; hence $n_{3,0}^{e}=3$,

The calculation of $n_{3,0}^{r}$ is more intricate. Here we must study the rational cubic curves in the fibers of $g$. A cubic curve is rational only if it is singular. So we investigate the locus of singular cubic curves. This is seen to be a plane curve of degree 36. But this curve is singular. In fact, the curve has nodes at each of the 177 cubics that we found earlier that factor into a line and a conic. This is because such curves have 2 singularities, which can be smoothed independently, yielding 2 distinct tangent directions in the space of singular curves. In addition, there are 216 cusps 14

It will be illuminating to generalize this situation before continuing the calculation. We suppose that we have a family of nodal elliptic curves with parameter space of curve $B$ of arithmetic genus $p_{a}$, containing $\delta$ nodes and $\kappa$ cusps. The nodes parameterize 2-nodal elliptic curves (which necessarily split up into 2 smooth rational curves, meeting twice), and the cusps parameterize cuspidal curves. In our example, $p_{a}=(36-1)(35-1) / 2=595$, while $\delta=177$ and $\kappa=216$.

Resolving the singularities of $B$, we get a curve $\tilde{B}$ of genus $p_{a}-\delta-\kappa$; and the computation from [21] shows that we must make a correction for the cusps, finally obtaining the Gromov-Witten invariant $c_{1}\left(\Omega^{1}(\tilde{B})\right)-\kappa=2\left(p_{a}-\delta-\kappa\right)-2-\kappa$, which simplifies to $2 p_{a}-2 \delta-3 \kappa-2$. In our example, this gives $n_{3,0}^{r}=186$.

This is not coincidentally the negative of the Euler characteristic of the target space $\tilde{X}$. We calculate the Euler characteristic by decomposing $\tilde{X}$. Again we generalize, assuming that there is a map $f: \tilde{X} \rightarrow S$, where $S$ is a smooth complex surface. We suppose that the general fiber of $f$ is a smooth elliptic curve, while over some curve $B$ contained in the surface, the fiber degenerates in the manner described above. We keep the notation $p_{a}, \delta, \kappa$ as before.

We divide $S$ into four pieces: $S-B$, the complement of the nodes and cusps in $B$, the nodes, and the cusps. We can then divide $\tilde{X}$ into four corresponding pieces, the inverse images of these four pieces via $f$. We will calculate the Euler characteristic of these four pieces.

We start the calculation by a preliminary calculation on $B$. We obtain a node by pinching a 1-cycle to a point, and a cusp by pinching two 1-cycles. If we then remove the resulting singularities, we compute that the Euler characteristic of the complement of the nodes and cusps in $B$ is $2-2 p_{a}+\delta+2 \kappa-(\delta+\kappa)$, which simplifies to $2-2 p_{a}+\kappa$.

14 The statements about the degree and number of cusps can for instance be checked by the standard technique of considering pairs $(C, p)$ where $C$ is one of the cubic curves and $p \in C$, then projecting onto $C$. The Schubert code for these calculations is available upon request. 
We also need to observe that the Euler characteristic of a smooth elliptic curve, the nodal curves, the 2-nodal curves, and the cuspidal curves have respective Euler characteristics $0,1,2,2$.

We can obtain the Euler characteristic of each piece by multiplying the Euler characteristics of the base and the fiber, then summing over all pieces. The result is $0+\left(2-2 p_{a}+\kappa\right)(1)+\delta(2)+\kappa(2)$, which simplifies to $2-2 p_{a}+2 \delta+3 \kappa$. This is plainly the negative of the Gromov-Witten invariant obtained above. This phenomenon occurs in several of the the examples discussed in [22,46].

For $n_{3,1}^{e}$, we claim that a curve $C$ with $C \cdot J_{1}=3$ and $C \cdot J_{2}=1$ is reducible. Suppose it were irreducible. Then $J_{2}$ restricts to a degree 1 bundle on $C$, which has a 1 dimensional space of global sections. Since $J_{2}$ has a 3 dimensional space of global sections (spanned by $\left.y_{1}, y_{2}, y_{3}\right)$, the kernel of the restriction map is at least 2 dimensional. This constrains $C$ to lie in a fiber of $g$. But we have already noted that the fibers of $g$ are of type $(3,0)$, so this is impossible. Looking at our prior discussion of curves of type $(a, b)$ with $a \leq 3$ and $b \leq 1$, we see that the only possibility is $C=C_{1} \cup C_{2}$, where the $C_{i}$ are of type $(3,0)$ and $(0,1)$ respectively. Such curves are parameterized by $E$ - take the fiber $f$ through the point $p \in E$ union the fiber of $g$ through the image of $p$ in $\mathbf{P}^{2}$. Since $c_{2}(E)=4$, this gives $n_{3,1}^{e}=4$.

Finally, we can easily see that $n_{4,0}=n_{5,0}=177$. For each of the 177 line-conic pairs, we have two families of degenerate instantons. These are analyzed by the method in the appendix to [8]; since our situation is simpler, we will content ourselves with sketching the construction. The first consists of maps from $\mathbf{P}^{1}$ to the conic, with lines bubbling off at each of the two nodes. These are degenerate instantons of type $(4,0)$. The other family comes from maps from $\mathbf{P}^{1}$ to the line, with conics bubbling off at each of the nodes. These are degenerate instantons of type $(5,0)$.

One can in fact verify that there is a non-zero contribution to $n_{i, 0}$ for all $i$ by using stable maps [56] as the method to compactify the space of instantons. All stable maps needed will restrict to degree 1 maps on each irreducible component.

If $i=3 k$, we can take a tree $C=C_{1} \cup \cdots \cup C_{k}$ of rational curves, and map the intersection points of the components to the nodes of any of the nodal cubic curves described above, with the rule that if $C_{j-1}$ maps to one branch of a nodal cubic near $p=C_{j-1} \cap C_{j}$, then $C_{j}$ maps to the other branch near $p$.

If $i=3 k+1$, we take $C=C_{1} \cup \cdots \cup C_{2 k+1}$, mapping $C_{2 j-1}$ to one of the lines found above, and mapping $C_{2 j}$ to the intersecting conic. Each $C_{l-1} \cap C_{l}$ maps to one of the two intersection points of a line and a conic. If $l<2 k+1$, then $C_{l} \cap C_{l+1}$ maps to the other intersection point.

The case $i=3 k+2$ is similar, this time mapping $C_{2 j-1}$ to conics and $C_{2 j}$ to lines. 


\section{2. $\mathbf{P}^{4}(1,1,1,1,3)$}

We desingularize the weighted projective space in this case by using an auxiliary $\mathbf{P}^{3}$ with coordinates $\left(y_{1}, y_{2}, y_{3}, y_{4}\right)$ and define

$$
\tilde{\mathbf{P}^{4}} \subset \mathbf{P}^{4}(1,1,1,1,3) \times \mathbf{P}^{3}
$$

by the equations

$$
x_{i} y_{j}=x_{j} y_{i}, \quad i=1, \ldots, 4 .
$$

The exceptional locus is clearly a copy of $\mathbf{P}^{3}$. The proper transform of a general degree 7 hypersurface is seen to intersect this locus in a linear hypersurface, i.e. a projective plane. This can be seen by direct calculation, the essential point being the presence of the monomials $x_{i} x_{5}^{2}$ for $i=1, \ldots, 4$ which are linear in the variables $x_{1}, \ldots, x_{4}$.

Now $J_{1}$ and $J_{2}$ are respectively the classes of degree 3 and degree 1 polynomials. This leads to the equality $J_{1}=3 J_{2}+E$, where $E$ is the class of the exceptional $\mathbf{P}^{2}$. So if a curve $C$ with $C \cdot J_{i}=a_{i}$ and $a_{1}<3 a_{2}$, then a component of $C$ necessarily lies on $E$.

To compute $n_{0, j}^{r}$, we see that these curves lie on $E$, and have degree $j$ as a curve on $E$. Since lines are parameterized by $\mathbf{P}^{2}$, we get $n_{0,1}^{r}=3$. Since conics are parameterized by $\mathbf{P}^{5}$, we get $n_{0,2}^{r}=-6$. Elliptic curves are similar; the lowest degree possible is cubic, so $n_{0, j}^{e}=0$ for $j=1,2$, while $n_{0,3}^{e}=-10$ since plane cubics are parameterized by a $\mathbf{P}^{9}$.

To compute $n_{1,0}^{r}$, we note that a curve $C$ with $C \cdot J_{2}=0$ maps to a point under the projection to $\mathbf{P}^{3}$, since the projection map is defined by the sections of $J_{2}$. We write the equation of the proper transform of the hypersurface as $f_{7}(y)+f_{4}(y) x_{5}+f_{1}(y) x_{5}^{2}$, where the $f_{j}(y)$ are homogeneous polynomials of degree $j$ in $y_{1}, \ldots, y_{4}$. The 28 values of $y$ for which $f_{7}(y)=f_{4}(y)=f_{1}(y)=0$ yield a $\mathbf{P}^{1}$ in the hypersurface (since $x_{5}$ may be arbitrary); this curve is easily seen to have $J_{1}$-degree 1 . This gives $n_{1,0}^{r}=28$. This geometry also implies that $n_{i, 0}^{r}=0$ for $i>1$. Each such curve $C$ satisfies $C \cdot E=C \cdot J_{1}-3 C \cdot J_{2}=1$, so that $C \cap E$ is a point $p$. We can get reducible curves with $C \cdot J_{1}=1$ and $C \cdot J_{2}=1$ (resp. 2) by taking the union of $C$ with a line (resp. a conic) in $E$ passing through $p$. Since lines (resp. conics) passing through $p$ are parameterized by $\mathbf{P}^{1}\left(\right.$ resp. $\left.\mathbf{P}^{4}\right)$, we get $n_{1,1}^{r}=28(-2)=-56$ and $n_{1,2}^{r}=28(5)=140$. Also, we can construct rational curves of type $(2,1)$ by taking a pair of curves of type $(1,0)$ and taking their union with the unique line in $E$ passing through the respective intersection points of these two curves with $E$. Thus $n_{2,1}^{r}=\left(\begin{array}{c}28 \\ 2\end{array}\right)=378$.

Irreducible elliptic curves $C$ of type $(5,2)$ satisfy $C \cdot E=-1$, so these lie on $E$. Such curves do not exist, since we know that the plane curves in $E$ have type $(0, j)$ for some $j$. The only possible reducible curves are obtained as follows. Consider one of the rational curves $C_{1}$ of type $(5,1)$. These meet $E$ in 2 points, which may be joined by the unique line $C_{2}$ (of type $(0,1)$ ) meeting these points. The curve $C=C_{1} \cup C_{2}$ is of type $(5,2)$, and is elliptic. This verifies $n_{5,2}^{e}=n_{5,1}^{r}$. 
Considering elliptic curves of type $(i, 3)$ for $i<9$, we see that such curves have a component contained in $E$. We see that if $i<5$, this component must be one of the $(0,3)$ elliptic curves considered above, and there are $i$ additional components, each rational curves of type $(1,0)$. This gives $n_{i, 3}^{e}=\left(\begin{array}{c}28 \\ i\end{array}\right) \cdot(-1)^{i-1}(10-i)$, since plane cubics passing through $i$ fixed points are parameterized by a $\mathbf{P}^{9-i}$. These are in agreement with the invariants produced from the instanton expansion. This does not hold for $5 \leq i<9$, due to the presence of reducible elliptic curves containing a rational component of type $(5,1)$.

\section{3. $\mathbf{P}^{4}(1,2,3,3,4)$}

We start by describing the geometry of the toric variety $\mathbf{P}_{\Delta^{*}}$, especially its Kähler cone. It will be convenient in the sequel to consult the following table.

$\begin{array}{ccccccc}\nu^{*(1)} & (-2,-3,-3,-4) & 1 & 0 & 1 & 0 & -1 \\ \nu^{*(2)} & (1,0,0,0) & 0 & 1 & 0 & 1 & -1 \\ \nu^{*(3)} & (0,1,0,0) & 0 & -1 & 1 & 0 & 0 \\ \nu^{*(4)} & (0,0,1,0) & 0 & -1 & 1 & 0 & 0 \\ \nu^{*(5)} & (0,0,0,1) & 1 & 0 & 0 & 0 & 0 \\ \nu^{*(6)} & (0,-1,-1,-1) & 1 & 0 & 0 & -2 & 1 \\ \nu^{*(7)} & (-1,-1,-1,-2) & 0 & 3 & -2 & 0 & 0 \\ \nu^{*(8)} & (-1,-2,-2,-2) & -2 & 0 & 0 & 1 & 0 \\ \nu^{*(9)} & (-1,-2,-2,-3) & 0 & -2 & 0 & 0 & 1\end{array}$

Here, the five columns on the right come from the representation of the Mori cone found in (3.18) (the first coordinate of the earlier representation, corresponding to the origin, is not needed in the current context). This representation gives a basis for the divisor class group of the toric variety; the coordinates of the classes of the divisors $D_{i}$ given by the equations $x_{i}=0$ (corresponding to the edges $\nu^{*(i)}$ ) can be read off horizontally in the above.

Now we find the edges of the Kähler cone by taking the dual basis (recall that the Kähler cone is dual to the Mori cone). We find linear combinations of the generators $D_{1}, \ldots, D_{9}$ such that in the coordinates given by the corresponding rows, we get the five standard basis vectors of $\mathbf{Z}^{5}$. There are many ways of expressing these in terms of the $D_{i}$; here is one choice.

$$
\begin{aligned}
& J_{1}=D_{5} \\
& J_{2}=D_{2}+D_{5}+D_{6}+D_{8} \\
& J_{3}=D_{2}+D_{3}+D_{5}+D_{6}+D_{8} \\
& J_{4}=2 D_{5}+D_{8} \\
& J_{5}=3 D_{5}+D_{6}+2 D_{8}
\end{aligned}
$$


Toric varieties can also be described as quotients of torus actions. We see that the toric variety $\mathbf{P}_{\Delta^{*}}$ is naturally identified with $\left(\mathbb{C}^{9}-F\right) / \mathbb{C}^{* 5}, 15$ where

$$
\begin{gathered}
F=\left\{x_{2}=x_{7}=0\right\} \cup\left\{x_{2}=x_{8}=0\right\} \cup\left\{x_{5}=x_{9}=0\right\} \cup\left\{x_{6}=x_{7}=0\right\} \cup\left\{x_{6}=x_{9}=0\right\} \\
\cup\left\{x_{7}=x_{8}=0\right\} \cup\left\{x_{8}=x_{9}=0\right\} \cup\left\{x_{1}=x_{2}=x_{5}=0\right\} \cup\left\{x_{1}=x_{3}=x_{4}=0\right\} \\
\cup\left\{x_{3}=x_{4}=x_{6}=0\right\} \cup\left\{x_{3}=x_{4}=x_{8}=0\right\} \cup\left\{x_{1}=x_{5}=x_{6}=0\right\} .
\end{gathered}
$$

The only singular point of the toric variety $\mathbf{P}_{\Delta^{*}}$ is the point $x_{2}=x_{3}=x_{4}=x_{9}=0$ corresponding to the determinant 3 cone spanned by $\nu^{*(2)}, \nu^{*(3)}, \nu^{*(4)}, \nu^{*(9)}$. Since the general Calabi-Yau hypersurface $X$ misses this point, $X$ is smooth.

We now identify the classes of curves $C_{1} \ldots, C_{5}$ which generate the Mori cone. These curves by definition are dual to the divisors $J_{i}$ via the intersection pairing. The intersection numbers $C_{i} \cdot D_{j}$ can be read from the last five columns of (4.3), at least up to a scalar multiple of each column. If the intersection number is negative, then necessarily $C_{i}$ is contained in $D_{j}$. So we see that $C_{1} \ldots C_{5}$ are respectively contained in the divisor $D_{8}$, the empty set $D_{3} \cap D_{4} \cap D_{9}$, the divisor $D_{7}$, the divisor $D_{6}$, and the curve $D_{1} \cap D_{2}$.

We immediately observe that the Mori cone of $X$ differs from the Mori cone of the toric variety, since $C_{2}$ is not present on $X$. For comparison, the locus $x_{3}=x_{4}=x_{9}=0$ is a curve on the toric variety $\mathbf{P}_{\Delta^{*}}$, whose intersection numbers with the $D_{i}$ are given by $1 / 3$ of the corresponding column of (4.3). The fractional intersection numbers are not surprising, since $C_{2}$ passes through the singular point of $\mathbf{P}_{\Delta^{*}}$. The fact that $D_{3} \cap D_{4} \cap D_{9}$ has dimension less than 1 is to be expected on dimensional grounds; but can be verified conclusively by explicitly writing down the general equation for $X$, substituting $x_{3}=x_{4}=x_{9}=0$, and noting that vanishing of the remaining term would imply that the point is contained in the disallowed set $F$. So this set is in fact empty.

We next set out to identify the Mori cone of $X$. Consider the class of a curve of type $C=\sum_{i} a_{i} C_{i}$. In the sequel, we will sometimes call this a curve of type $\left(a_{1}, \ldots, a_{5}\right)$. Suppose that this is the class of an effective curve on $X$. We claim that

$$
a_{1} \geq 0, a_{3} \geq a_{2} \geq 0, a_{4} \geq 0, a_{5} \geq 0 .
$$

The inequalities $C \cdot J_{i} \geq 0$ hold because $C$ is an effective curve on $\mathbf{P}_{\Delta^{*}}$ and the $J_{i}$ are in the Kähler cone. But $C \cdot J_{i}=a_{i}$. Now suppose that $a_{2}>a_{3}$. Without loss of generality, we may assume that $C$ is irreducible. Then $C \cdot D_{3}=C \cdot D_{4}=a_{3}-a_{2}<0$. Thus $C \subset D_{3} \cap D_{4}$. We have already seen that $D_{3} \cap D_{4} \cap D_{9}$ is empty. The intersection of $D_{3} \cap D_{4}$ with any

15 This and other calculations in this section were performed using an extensive collection of Maple procedures for toric varieties which has been written jointly by the second author and S.A. Strømme; a modified version of these will appear in the next release of Schubert. 
of $D_{1}, D_{6}$, or $D_{8}$ is empty as well, by consideration of the locus $F$. So we only have to show that $C \cdot D_{i}<0$ for at least one of $i=1,6,8,9$. But the system of inequalities in the $a_{i}$ deduced from the inequalities $C \cdot D_{i} \geq 0$ for $i=1,6,8,9$ together with $a_{i} \geq 0$ is readily seen to be inconsistent, justifying (4.4).

To show that the system (4.4) indeed defines the Mori cone, we first note that it is a simplicial cone spanned by $C_{1}, C_{2}+C_{3}, C_{3}, C_{4}, C_{5}$. So it suffices to show that all of these are classes of a curve on $X$. We denote these classes by $\tilde{C}_{1}, \ldots, \tilde{C}_{5}$.

Let $\tilde{J}_{i}, i=1, \ldots, 5$ be the dual basis of divisors (which will turn out to be the generators of the Kähler cone of $X$ ). This gives explicitly $\tilde{J}_{i}=J_{i}$ for $i=1,2,4,5$, and $\tilde{J}_{3}=J_{3}-J_{2}=D_{3}$.

We have already seen that if the respective curves $\tilde{C}_{1}, \tilde{C}_{3}, \tilde{C}_{4}$ exist, they lie in the divisors $D_{8}, D_{7}, D_{6}$ and $\tilde{C}_{5}$ lies in the curve $D_{1} \cap D_{2}$. By the same reasoning, we see that $\tilde{C}_{2}$ lies in the divisor $D_{9}$.

Let $\hat{\mathcal{J}}_{i}$ denote the wall of the Kähler cone obtained by taking non-negative linear combinations of all of the $\tilde{J}_{k}$ except $\tilde{J}_{i}$. According to [57, these walls consist of divisor classes which contract $\tilde{C}_{i}$ (i.e. the divisors intersect each curve in the locus trivially). Let us find these loci. First, we identify all curves $x_{i}=x_{j}=0$ which meet the divisors in $\hat{\mathcal{J}}_{i}$ trivially. We may ignore the curves which are components of $F$. Direct calculation gives

$$
\begin{array}{cc}
1 & \left(x_{3}=x_{8}=0\right) \cup\left(x_{4}=x_{8}=0\right) \\
2 & \left(x_{3}=x_{9}=0\right) \cup\left(x_{4}=x_{9}=0\right) \\
3 & \left(x_{5}=x_{7}=0\right) \cup\left(x_{7}=x_{9}=0\right) \\
4 & \left(x_{1}=x_{6}=0\right) \cup\left(x_{3}=x_{6}=0\right) \cup\left(x_{4}=x_{6}=0\right) \cup\left(x_{5}=x_{6}=0\right) \\
5 & \left(x_{1}=x_{2}=0\right)
\end{array}
$$

The above table identifies interesting curves, which leads to the following results.

The divisors of $\hat{\mathcal{J}}_{1}$ blow down the fibers of a base point free pencil on $x_{8}=0$ generated by $x_{3}, x_{4}$ (base point free since $x_{3}=x_{4}=x_{8}=0$ is a component of $F$ ). The fibers are calculated to be rational curves of type $(1,0,0,0,0)$. This verifies $n_{1,0,0,0,0}=-2$ and $n_{j, 0,0,0,0}=0$ for $j>1$.

The divisors of $\hat{\mathcal{J}}_{2}$ blow down the fibers of a base point free pencil on $x_{9}=0$ generated by $\left(x_{3}, x_{4}\right.$ ) (while $x_{3}=x_{4}=x_{9}=0$ is non-empty on $\mathbf{P}_{\Delta^{*}}$ since it is not a component of $F$, it is in fact empty on $X)$. The fibers are calculated to be rational curves of type $(0,1,1,0,0)$. This verifies $n_{0,1,1,0,0}=-2$ and $n_{0, j, j, 0,0}=0$ for $j>1$.

Similarly, the divisors of $\hat{\mathcal{J}}_{3}$ blow down the fibers of a base point free pencil on $x_{7}=0$ generated by $x_{5}, x_{9}$. Note that while $\left(x_{5}, x_{9}\right)$ scale differently, the description of $x_{7}=0$ as a toric variety shows that $x_{5}=0$ and $x_{9}=0$ are linearly equivalent on $x_{7}=0$. The fibers are calculated to be rational curves of type $(0,0,1,0,0)$. This verifies $n_{0,0,1,0,0}=-2$ and $n_{0,0, j, 0,0}=0$ for $j>1$.

The divisors of $\hat{\mathcal{J}}_{4}$ blow down the fibers of the base point free pencil on $x_{6}=0$ generated by $\left(x_{3}, x_{4}\right)$. The fibers are calculated to be rational curves of type $(0,0,0,1,0)$. 
This verifies $n_{0,0,0,1,0}=-2$ and $n_{0,0,0, j, 0}=0$ for $j>1$. Note that the curves $x_{1}=x_{6}=0$ and $x_{5}=x_{6}=0$ are actually disconnected unions of 3 and 4 fibers respectively, so contribute nothing new.

The divisors of $\hat{\mathcal{J}}_{5}$ blow down the curve $x_{1}=x_{2}=0$, which is easily seen to have three irreducible connected components, each of which is of type $(0,0,0,0,1)$. This verifies $n_{0,0,0,0,1}=3$ and $n_{0,0,0,0, j}=0$ for $j>1$.

We can also verify several other numbers. For example, degenerate instantons can be formed by taking the unions of transversely intersecting irreducible rational curves which have no closed loops (hence still have genus 0 ). Here is a short list.

Each of the 3 curves of type $(0,0,0,0,1)$ meets each of $x_{6}=0$ and $x_{9}=0$ once. So there is a unique fiber of either or both of these surfaces that can be joined to each of these 3 curves to get a rational curve (a glance at $F$ shows that $x_{6}=0$ and $x_{9}=0$ are disjoint). This verifies $n_{0,0,0,1,1}=n_{0,1,1,0,1}=n_{0,1,1,1,1}=3$.

Now consider the curve $C$ given by $x_{7}=x_{9}=0$. This is one of the $\hat{\mathcal{J}}_{3}$-exceptional curves already considered, so is of type $(0,0,1,0,0)$. The curve $C$ meets each of the exceptional fibers of type $(0,1,1,0,0)$ on $x_{9}=0$ in one point, so can be joined to each of these fibers. We again get a family of curves parameterized by $\mathbf{P}^{1}$. This verifies $n_{0,1,2,0,0}=$ -2 . Now each of the 3 curves of type $(0,0,0,0,1)$ can be joined to a unique element of this family; and then again a unique fiber of $x_{6}=0$ can be joined afterwards. This verifies $n_{0,1,2,0,1}=n_{0,1,2,1,1}=3$.

Note also that the fibrations on $x_{6}=0$ and $x_{8}=0$ are defined by the same pencil $\left(x_{3}, x_{4}\right)$, and that the corresponding fibers meet in one point. Taking unions again gives a family of curves parameterized by $\mathbf{P}^{1}$. Then each of the 3 curves of type $(0,0,0,0,1)$ can be joined to precisely one of these, yielding a rational curve (each such curve meets $x_{6}=0$ once but is disjoint from $\left.x_{8}=0\right)$. This verifies $n_{1,0,0,1,0}=-2$ and $n_{1,0,0,1,1}=3$.

We next observe that curves of type $(0,2,3,0,0)$ are restrictions to $x_{9}=0$ of the divisors linearly equivalent to $J_{2}$. These restrictions are parameterized by $\mathbf{P}^{3}$ (explicitly, the divisors are defined by linear combinations of $\left.x_{3}^{2} x_{7}, x_{3} x_{4} x_{7}, x_{4}^{2} x_{7}, x_{2} x_{5} x_{6} x_{8}\right)$. This gives $n_{0,2,3,0,0}=-4$. Each of the 3 curves of type $(0,0,0,0,1)$ meets $x_{9}=0$ in one point; the divisors of $J_{2}$ passing through this point (the only ones that can be joined to get a connected curve) are parameterized by $\mathbf{P}^{2}$. This gives $n_{0,2,3,0,1}=3 \cdot 3=9$. Similarly we can add on two curves (which restricts divisors of $J_{2}$ to a $\mathbf{P}^{1}$ ) or all three (uniquely specifying the divisor of $J_{2}$ ). This verifies $n_{0,2,3,0,2}=3 \cdot(-2)=-6$ and $n_{0,2,3,0,3}=1$, while $n_{0,2,3,0, j}=0$ for $j>3$.

Finally we observe that curves of type $(0,3,4,0,0)$ are restrictions to $x_{9}=0$ of the divisors linearly equivalent to $J_{2}+J_{3}$. These restrictions are parameterized by $\mathbf{P}^{5}$ (explicitly, the divisors are defined by linear combinations of $f_{3}\left(x_{3}, x_{4}\right) x_{7}, x_{2} x_{3} x_{5} x_{6} x_{8}, x_{2} x_{4} x_{5} x_{6} x_{8}$, and $x_{1} x_{5}^{2} x_{6} x_{8}^{2}$, where $f_{3}\left(x_{3}, x_{4}\right)$ is a homogeneous cubic; note that when restricted to $X \cap\left(x_{9}=0\right)$, the last monomial becomes a linear combination of the others). This gives 
$n_{0,3,4,0,0}=-6$, and proceeding as before, we also get $n_{0,3,4,0,1}=3 \cdot 5=15, n_{0,3,4,0,2}=$ $3 \cdot(-4)=-12$, and $n_{0,3,4,0,3}=3$.

The calculation of the Mori cone of $X$ shows that the true large complex structure coordinates are $q_{1}, q_{2} q_{3}, q_{3}, q_{4}, q_{5}$. In other words, the boundary divisor $q_{3}=0$ in the moduli space constructed from toric geometry gets blown down, slightly changing the geometry of the large complex structure limit. The instanton expansions remain valid, the only change being that we get the more precise result that the instanton expansions must be power series in $q_{1}, q_{2} q_{3}, q_{3}, q_{4}, q_{5}$, agreeing with our calculations.

\section{Applications and Generalizations}

\subsection{Non-Landau-Ginzburg cases}

The classification of the three dimensional polyhedra appears as the natural generalization of the work of [25]. We will not solve this complicated combinatorial problem here, but rather give the first examples of reflexive pairs of polyhedra, which do not come from the list of [25] nor from moddings of the examples in that list [58]. The important observation in finding such examples is the fact that one can systematically modify a given polyhedron by moving one corner to a new position, in such a fashion that the new polyhedron remains reflexive. We can then use the general methods for determining the Picard-Fuchs equations for reflexive polyhedra, which were developed in the previous section.

As an example of this phenomenon let us consider some of the two moduli models of table 3.1 and move one of the vertices to a new position without destroying reflexivity. In table 5.1 we list models constructed by moving the point $\nu^{*(1)}$ such that the resulting Calabi-Yau spaces have $h_{11}<4$. In $\left.a\right)-c$ ), the original location for $\nu^{*(1)}$ is $\left(-k_{2},-k_{3},-k_{4},-k_{5}\right)$, see (2.4). There are no such natural coordinates for the model in table 5.1.d); a choice of coordinates has been made here 16

16 We are grateful to Maximilian Kreuzer for checking that the models marked with a star in table 5.1 do not appear as a result of various moddings [58] of the models in [25]. 


\begin{tabular}{|c|r|r|r|}
\hline$\nu^{*(1)}$ & $\chi$ & $h_{11}$ & $h_{21}$ \\
\hline$(-1,-1,-2,-2)$ & -186 & $2(0)$ & $95(0)$ \\
$(-1,-1,-1,-2)^{*}$ & -180 & $2(0)$ & $92(0)$ \\
$(-1,-1,-1,-1)$ & -168 & $2(0)$ & $86(0)$ \\
$(-1,-1,0,0)^{*}$ & -162 & $2(0)$ & $83(0)$ \\
\hline$(-1,-1,-3,3)$ & -324 & $3(0)$ & $165(0)$ \\
$(-1,-1,3,3)^{*}$ & -216 & $3(0)$ & $111(0)$ \\
$(-2,-1,-2,0)^{*}$ & -156 & $3(0)$ & $81(0)$ \\
$(-1,-2,0,0)$ & -144 & $3(0)$ & $75(1)$ \\
$(-3,-2,-2,2)^{\diamond}$ & -120 & $3(0)$ & $63(1)$ \\
\hline
\end{tabular}

b)

\begin{tabular}{|c|r|r|r|}
\hline$\nu^{*(1)}$ & $\chi$ & $h_{11}$ & $h_{21}$ \\
\hline$(-1,-2,-2,-6)$ & -252 & $2(0)$ & $128(0)$ \\
$(-1,-1,-1,-3)$ & -240 & $2(0)$ & $122(0)$ \\
$(-1,-1,-1,-2) *$ & -200 & $2(0)$ & $102(0)$ \\
$(-1,-1,-2,-3)$ & -208 & $2(0)$ & $106(0)$ \\
$(-1,-1,-1,-1) \diamond$ & -176 & $2(0)$ & $90(0)$ \\
$(-1,-1,-1,0)$ & -168 & $2(0)$ & $86(0)$ \\
\hline$(-1,-1,-3,-6)$ & -324 & $3(0)$ & $165(1)$ \\
$(-1,-2,-2,-4) *$ & -192 & $3(0)$ & $99(0)$ \\
$(-1,-2,-2,-2) *$ & -156 & $3(0)$ & $81(0)$ \\
$(-1,-2,-2,0)$ & -144 & $3(0)$ & $75(2)$ \\
\hline
\end{tabular}

\begin{tabular}{|c|r|r|r|}
\hline$\nu^{*(1)}$ & $\chi$ & $h_{11}$ & $h_{21}$ \\
\hline$(0,0,-1,-1)$ & -168 & $2(0)$ & $86(0)$ \\
\hline$(-1,0,-2,-2)$ & -168 & $3(0)$ & $87(0)$ \\
$(-1,0,-2,0)^{*}$ & -172 & $3(0)$ & $89(0)$ \\
c) $(-1,-1,-2,-3)$ & -132 & $3(0)$ & $69(3)$ \\
\hline
\end{tabular}

d)

\begin{tabular}{|c|r|c|r|}
\hline$\nu^{*(1)}$ & $\chi$ & $h_{11}$ & $h_{21}$ \\
\hline$(-1,-1,-1,-1)^{*}$ & -152 & $3(0)$ & $79(1)$ \\
$(-1,-1,0,0)$ & -144 & $3(0)$ & $75(1)$ \\
$(-1,-1,-1,0)$ & -132 & $3(0)$ & $69(3)$ \\
$(-1,-1,-2,-3)^{*}$ & -124 & $3(0)$ & $65(3)$ \\
$(-1,-1,0,0) \diamond$ & -112 & $3(0)$ & $59(6)$ \\
$(-2,-1,-1,1) \diamond$ & -108 & $3(0)$ & $57(4)$ \\
\hline
\end{tabular}

Table 5.1: Calabi-Yau hypersurfaces, $a)-d$ ) with $h_{11}=2,3$ in toric varieties, which derive from the polyhedron of $X_{7}(1,1,1,2,2), X_{7}(1,1,1,1,3), X_{12}(1,1,3,3,4)$ and $X_{28}(2,2,3,7,14)$ by moving the point $\nu^{*(1)}$. Examples which do not appear in the list of [25] are marked with a star. Models for which the Hodge numbers agree with that of a Landau-Ginzburg model with more than five fields are marked with a $\diamond$, although it is not clear that this makes the theories the same.

The generators of the Mori cone for the second case in the table 5.1.a), which is a new two moduli example, are

$$
l^{(1)}=(-3,0,0,0,1,1,1), \quad l^{(2)}=(0,1,1,1,-1,0,-2)
$$

In this case the third and second order Picard-Fuchs operators follow in a very simple manner by factorization

$$
\begin{aligned}
& \mathcal{L}_{1}=\left(\theta_{2}-\theta_{1}\right)\left(\theta_{1}-2 \theta_{2}\right)-3\left(3 \theta_{1}-2\right)\left(3 \theta_{1}-1\right) z_{1} \\
& \mathcal{L}_{2}=\theta_{2}^{3}-\left(2 \theta_{2}-\theta_{1}-1\right)\left(2 \theta_{2}-\theta_{1}-2\right)\left(\theta_{1}-\theta_{2}+1\right) z_{2},
\end{aligned}
$$

The discriminant and the Yukawa couplings are calculated as

$$
\Delta=\left(1-27 z_{1}\right)^{3}+27 z_{1} z_{2}\left(1-540 z_{1}+5832 z_{1}^{2}+11664 z_{1}^{2} z_{2}\right)
$$




$$
\begin{gathered}
K_{111}=\frac{3\left(7-54 z_{1}-216 z_{1} z_{2}\right)}{z_{1}^{3} \Delta}, \quad K_{112}=\frac{9 z_{2}\left(1-27 z_{1}+36 z_{1}-32 z_{1} z_{2}\right)}{z_{1}^{2} \Delta} \\
K_{122}=\frac{3\left(\left(1-27 z_{1}\right)^{2}+54 z_{1} z_{2}+2916 z_{1}^{2} z_{2}\right)}{z_{1} z_{2}^{2} \Delta} \quad K_{222}=\frac{81 z_{1}\left(1+216 z_{1}\right)}{z_{2}^{2} \Delta,}
\end{gathered}
$$

Instead of (2.15), which applies only to hypersurfaces in weighted projective spaces, we use the formulas of 46]

$$
\begin{aligned}
& \int_{X} c_{2} \wedge J_{p}=\frac{1}{2} \sum_{m, n}\left(\sum_{i} l_{i}^{(m)} l_{i}^{(n)}\right) K_{J_{p} J_{m} J_{n}}, \\
& \chi=\int_{X} c_{3}=\frac{1}{3} \sum_{j, m, n}\left(\sum_{i} l_{i}^{(j)} l_{i}^{(m)} l_{i}^{(n)}\right) K_{J_{j} J_{m} J_{n}},
\end{aligned}
$$

which gives a relation between the evaluation of the second Chern class on $H^{(1,1)}$ as well as the Euler number with the intersection numbers. Formulas (5.4) apply to canonical resolved hypersurfaces and also to canonical resolved complete intersections in general toric varieties. Knowing that $\chi=-168$ we get from (5.4) the following normalization of the couplings $K_{J_{1} J_{1} J_{1}}=21, K_{J_{1} J_{1} J_{2}}=9, K_{J_{1} J_{2} J_{2}}=3, K_{J_{2} J_{2} J_{2}}=0$. From the first relation in (5.4) follows $\int c_{2} J_{1}=78$ and $\int c_{2} J_{2}=36$, which fixes $s_{1}=-\frac{15}{2}, s_{2}=-4$. The fact that $n_{1,0}^{e}$ vanishes enforces $r_{0}=-1 / 6$, and leads to predictions for the instantons, as given in Table 5.2.

\begin{tabular}{|l|rrrrrrr|}
\hline$n_{i, j}$ & $j=0$ & $j=1$ & $j=2$ & $j=3$ & $j=4$ & $j=5$ & $j=6$ \\
\hline$n_{0, j}^{r}$ & 0 & $\forall j$ & & & & & \\
$n_{0, j}^{e}$ & 0 & $\forall j$ & & & & & \\
\hline$n_{1, j}^{r}$ & 180 & 27 & 0 & $\forall j>1$ & & & \\
$n_{1, j}^{e}$ & 0 & $\forall j$ & & & & & \\
\hline$n_{2, j}^{r}$ & 180 & 6804 & -54 & 0 & $\forall j>2$ & & \\
$n_{2, j}^{e}$ & 0 & $\forall j$ & & & & & \\
\hline$n_{3, j}^{r}$ & 180 & 138510 & 4860 & 243 & 0 & $\forall j>3$ & \\
$n_{3, j}^{e}$ & 3 & 0 & 0 & -4 & 0 & 0 & 0 \\
\hline$n_{4, j}^{r}$ & 180 & 1478520 & 5103972 & -29520 & -1728 & 0 & 0 \\
$n_{4, j}^{e}$ & 0 & -54 & 6804 & 540 & 135 & 0 & 0 \\
\hline
\end{tabular}

Table 5.2 The invariants of rational and elliptic curves of degree $(i, j), n_{i, j}^{r}$ and $n_{i, j}^{e}$ respectively, for the non-LG two moduli case described above.

\subsection{Mirror nesting of the moduli spaces}

In [21,22] it was realized that certain models when restricted to a specific codimension one surface of the Kähler moduli space are birationally equivalent to a different 
Calabi-Yau manifold. The models in question have $h^{1,1}=2$ and a $Z_{2}$ curve singularity, $X_{8}(2,2,1,1,1)_{-168}^{2,86}, X_{12}(1,1,2,2,6)_{-252}^{2,128}, X_{12}(1,2,2,3,4)_{-144}^{2,74}, X_{14}(1,2,2,2,7)_{-240}^{2,122}$ There exists a one-dimensional subspace in the Kähler moduli space, which corresponds to the following one Kähler moduli Calabi-Yau spaces $X_{4,2}(1,1,1,1,1,1)_{-176}^{1,89}$, $X_{6,2}(1,1,1,1,1,3)_{-256}^{1,129}, X_{6,4}(1,1,1,2,2,3)_{-156}^{1,79}$ and $X_{8}(1,1,1,1,4)_{-296}^{1,149}$ respectively. In particular the relation manifests itself in the following relation between the topological invariants, $\sum_{d_{D}} n_{d_{J}, d_{D}}=n_{d_{J}}$ [21].

In the last case the Calabi-Yau manifold with the one dimensional Kähler deformation space is itself defined as a hypersurface of a toric variety given by a reflexive polyhedron This gives the following very simple interpretation 17 of the situation, namely that the polyhedra are nested into each other, i.e. $\Delta_{X_{8}(1,1,1,1,4)}^{*} \subset \Delta_{X_{14}(1,2,2,2,7)}^{*}$.

Let us now look in more detail at this nesting phenomenon and its implication on the moduli spaces and proceed with the model $X_{14}(1,2,2,2,7)$. In the case at hand $\Delta_{X_{14}(1,2,2,2,7)}^{*}$ is the convex hull of the following points

$$
\begin{aligned}
& \nu^{*(0)}=\left(\begin{array}{llll}
0, & 0, & 0, & 0
\end{array}\right)^{4}, \quad \nu^{*(1)}=(-2,-2,-2,-7)^{0}, \quad \nu^{*(2)}=\left(\begin{array}{cccc}
1, & 0, & 0, & 0
\end{array}\right)^{0}, \\
& \nu^{*(3)}=\left(\begin{array}{llll}
0, & 1, & 0, & 0
\end{array}\right)^{0}, \quad \nu^{*(4)}=\left(\begin{array}{llll}
0, & 0, & 1, & 0
\end{array}\right)^{0}, \quad \nu^{*(5)}=\left(\begin{array}{llll}
0, & 0, & 0, & 1
\end{array}\right)^{0},, \\
& \nu^{*(6)}=(-1,-1,-1,-3)^{1}, \quad \nu^{*(7)}=(-1,-1,-1,-4)^{3}, \quad \nu^{*(8)}=\left(\begin{array}{lll}
0, & 0, & 0,-1)^{3}
\end{array}\right.
\end{aligned}
$$

where we indicated the dimension of the lowest dimensional face the points lie on as upper index on the points. Since we include the codimension 1 points $\nu^{*(7)}, \nu^{*(8)}$ in the Laurent polynomial $P=\sum_{i=0}^{8} a_{i} \phi_{i}$ the two dimensional moduli space is redundantly parameterized. Part of the redundancy is removed by choosing representatives of the ring of deformations 18 . This can be done due to two relations of type (3.11) but at level one: $a_{0} \phi_{8}+2 a_{5} \phi_{0}+a_{6} \phi_{1}=\frac{d_{5}}{X_{4}}$ and $a_{0} \phi_{7}+2 a_{5} \phi_{5}+a_{6} \phi_{1}=\frac{d_{5}}{X_{1} X_{2} X_{3} X_{4}^{4}}$ with $d_{5}=$ $\left(1-\Theta_{1}-\Theta_{2}-\Theta_{3}+\Theta_{4}\right)$, which allows us either to set $a_{7}=a_{8}=0, a_{1} \stackrel{a_{8}}{=} a_{8}$ or $a_{1}=a_{7}=0$. In all cases the convex hull of the remaining Newton polyhedron is reflexive and as we argue below $\left(\Delta, \Delta^{*}\right)$ describes birationally equivalent manifolds in the three possible cases, although the polyhedra are different combinatorial objects. Note that the third possibility corresponds to the two moduli case $X_{7}(1,1,1,1,3)$ treated in section two.

Another possibility is to restrict oneself to a subspace of the moduli space, by forcing an additional coordinate $a_{i}$ to vanish. The only possible way to do this for the case at hand in such a way that the remaining Newton polyhedron stays reflexive is to set $a_{1}=a_{6}=a_{8}=0$, which leads to the Newton polyhedron describing the one dimensional moduli space of

17 This observation was also made by Shinobu Hosono, whom we thank for an email correspondence on this point.

18 The rest of the redundancy is removed by Euler type homogeneity conditions given by the generators of the Mori cone as usual. 
$X_{8}(1,1,1,1,4)_{-296}^{1,149}$ and explains the relation between the topological numbers of the two models. Let us summarize this situation in the table 5.3.

\begin{tabular}{|c|r|r|r|r|}
\hline$\left(\Delta, \Delta^{*}\right)$ & $\left(\Delta_{I}, \Delta_{I}^{*}\right)$ & $\left(\Delta_{I I}, \Delta_{I I}^{*}\right)$ & $\left(\Delta_{I I I}, \Delta_{I I I}^{*}\right)$ & $\left(\Delta_{I V}, \Delta_{I V}^{*}\right)$ \\
\hline$P$ & $a_{1}=a_{6}=a_{8}=0$ & $a_{1}=a_{8}=0$ & $a_{1}=a_{7}=0$ & $a_{7}=a_{8}=0$ \\
\hline$\Delta^{*}$ & $\operatorname{conv}(7,2,3,4,5)$ & $\operatorname{conv}(6,7,2,3,4,5)$ & $\operatorname{conv}(6,8,2,3,4,5)$ & $\operatorname{conv}(1,2,3,4,5)$ \\
\hline$\Delta$ & $\operatorname{conv}(1,2,3,4,5)$ & $\operatorname{conv}(1,2,6,7,8,9,10,11)$ & $\operatorname{conv}(1,2,6,7,8,12,13,14)$ & $\operatorname{conv}(1,2,6,7,8)$ \\
\hline$L G$ & $X_{8}(1,1,1,1,4)$ & --- & $X_{7}(1,1,1,1,3)$ & $X_{14}(1,2,2,2,7)$ \\
\hline$h^{1,1}$ & $1(0)$ & $2(0)$ & $2(0)$ & $2(0)$ \\
\hline$h^{2,1}$ & $149(0)$ & $122(0)$ & $122(0)$ & $122(15)$ \\
\hline$z_{1}$ & $\frac{a_{5}^{4} a_{2} a_{3} a_{4} a_{7}}{a_{0}^{8}}$ & $\frac{a_{2} a_{3} a_{4} a_{6}^{4}}{a_{0}^{4} a_{7}^{3}}$ & $-\frac{a_{2} a_{3} a_{4} a_{6}}{a_{0} a_{8}^{3}}$ & $-\frac{a_{2} a_{3} a_{4} a_{6}^{7}}{a_{0}^{7} a_{1}^{3}}$ \\
$z_{2}$ & & $-\frac{a_{5} a_{7}}{a_{0} a_{6}}$ & $\frac{a_{6} a_{8}}{a_{0}^{2}}$ & $\frac{a_{1} a_{5}}{a_{6}^{2}}$ \\
\hline
\end{tabular}

Table 5.3: The reflexive polyhedra inside $\Delta_{I V}^{*}=\Delta_{X_{14}(1,2,2,2,7)}^{*}$ as well as their duals. The polyhedra are specified as convex hulls of the points given in (5.5) and (5.2), with the row below indicating the corresponding LG-configuration if it exits. Note that $\Delta_{I}^{*} \subset \Delta_{I I}^{*} \subset \Delta_{I V}^{*}$ hence $\Delta_{I V} \subset \Delta_{I I} \subset \Delta_{I}$. Furthermore we list the number of Kähler and complex structure deformations of $X_{\Delta}$ (the number of non-algebraic deformations is indicated in parentheses) as well as the vanishing coefficient in the Laurent polynomial and the canonical large complex structure coordinates of $X_{\Delta}^{*}$, which are determined by the Mori cone. For each case the latter is generated by positive linear combinations of the generators of the Mori cone of $\mathbf{P}_{\Delta_{1}^{*}}$ which have vanishing entries at the places of the corresponding Laurent monomials.

The polyhedra $\Delta^{*}, \Delta$ in table 5.3 are specified as the convex hull of the points given in (5.5) and the following points inside of $\Delta_{X_{8}(1,1,1,1,4)}$

$$
\begin{aligned}
& \nu^{(1)}=(-1,-1,-1,-1)^{0}, \quad \nu^{(2)}=(-1,-1,-1, \quad 1)^{0}, \quad \nu^{(3)}=(-1,-1, \quad 7,-1)^{0}, \\
& \nu^{(4)}=(-1,7,-1,-1)^{0}, \quad \nu^{(5)}=(7,-1,-1,-1)^{0}, \quad \nu^{(6)}=(-1,-1, \quad 6,-1)^{1} \text {, } \\
& \nu^{(7)}=(-1, \quad 6,-1,-1)^{1}, \quad \nu^{(8)}=(6,-1,-1,-1)^{1}, \quad \nu^{(9)}=(-1,-1, \quad 3, \quad 0)^{1} \text {, } \\
& \nu^{(10)}=(-1, \quad 3,-1, \quad 0)^{1}, \quad \nu^{(11)}=(3,-1,-1, \quad 0)^{1}, \quad \nu^{(12)}=\left(\begin{array}{ll}
-1,-1, & 0, \quad 1
\end{array}\right) \text {, } \\
& \nu^{(13)}=(-1, \quad 0,-1, \quad 1) \quad \nu^{(14)}=\left(\begin{array}{lll}
0,-1,-1, & 1
\end{array}\right)
\end{aligned}
$$

This nesting phenomenon, which is ubiquitous among the reflexive polyhedra, has the following simple implications 19 for the moduli spaces of Kähler $\mathcal{M}_{1,1}\left(X_{\Delta_{1,2}}\right)$ and complex structure deformation $\mathcal{M}_{2,1}\left(X_{\Delta_{1,2}}\right)$ of the manifolds $X_{\Delta_{1,2}}$

$$
\begin{gathered}
\Delta_{1}^{*} \subset \Delta_{2}^{*} \Longleftrightarrow \Delta_{2} \subset \Delta_{1} \Longrightarrow \\
\mathcal{M}_{1,1}\left(X_{\Delta_{1}}\right) \subseteq \mathcal{M}_{1,1}\left(X_{\Delta_{2}}\right), \quad \mathcal{M}_{2,1}\left(X_{\Delta_{2}}\right) \subseteq \mathcal{M}_{2,1}\left(X_{\Delta_{1}}\right)
\end{gathered}
$$

19 We conjecture that $\mathcal{M}_{2,1}\left(X_{d}\right) \subset \mathcal{M}_{2,1}\left(X_{d_{1}, d_{2}}\right)$ is true for the first three examples given at the beginning of this section. 
If also $\operatorname{dim}\left(\mathcal{M}_{1,1}\left(X_{\Delta_{1}}\right)\right)=\operatorname{dim}\left(\mathcal{M}_{1,1}\left(X_{\Delta_{2}}\right)\right)$ and $\operatorname{dim}\left(\mathcal{M}_{2,1}\left(X_{\Delta_{1}}\right)\right)=\operatorname{dim}\left(\mathcal{M}_{2,1}\left(X_{\Delta_{2}}\right)\right)$ the manifolds will be birationally equivalent to each other. This is in fact the case for the last three examples in table 5.3.

Let us return to the example discussed in the beginning of this section and compare the manifolds associated to $\left(\Delta_{I}, \Delta_{I}^{*}\right)$ and $\left(\Delta_{I I}, \Delta_{I I}^{*}\right)$, which from now on will be denoted the I model and II model. The representation $\left(\Delta_{I I}, \Delta_{I I}^{*}\right)$ of table 5.3 is the most suitable for our purposes, because the restriction to the moduli space of the I model is simply given by $a_{6}=0$.

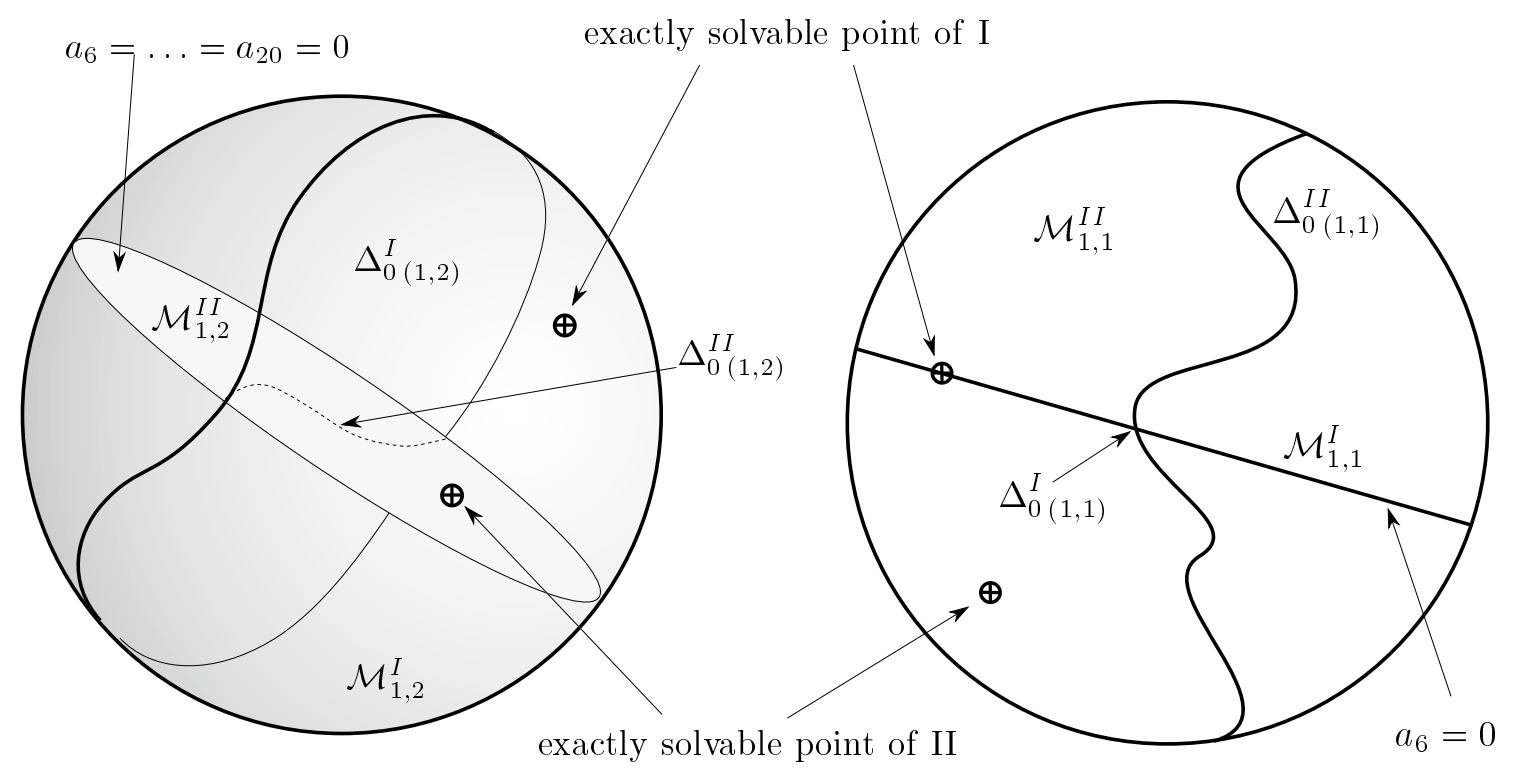

Complex moduli space $\operatorname{dim}_{\mathrm{c}}=149$

Kähler moduli space $\operatorname{dim}=2$

Fig.1: Schematic Picture of the mirror nesting of the moduli spaces for the I model and the II model

In the large Kähler structure variables $z_{1}, z_{2}$ the Picard-Fuchs system consists of the following two differential operators of second and third order

$$
\begin{aligned}
\mathcal{L}_{1} & =\theta_{1}^{2}\left(7 \theta_{1}-2 \theta_{2}\right)+7 z_{1}\left\{\left[\left(3 \theta_{1}-\theta_{2}+2\right)-2 z_{2}\left(12 \theta_{1}+\theta_{2}+6\right)\right]\left(3 \theta_{1}-\theta_{2}+1\right)\left(3 \theta_{1}-\theta_{2}\right)\right. \\
& +\left[\left(4 z_{2}^{2}\left(8 \theta_{1}+2 \theta_{2}+3\right)\left(3 \theta_{1}-\theta_{2}\right)-8 z_{2}^{3}\left(4 \theta_{1}+\theta_{2}+1\right)\left(4 \theta_{1}+\theta_{2}+3\right)\right]\left(8 \theta_{1}+5\right)\right\} \\
& -2 z_{2} \theta_{1}^{2}\left(4 \theta_{1}-\theta_{2}\right) \\
\mathcal{L}_{2} & =\theta_{2}\left(3 \theta_{1}+\theta_{2}\right)+z_{2}\left(4 \theta_{1}-\theta_{2}\right)\left(4 \theta_{1}+\theta_{2}+1\right),
\end{aligned}
$$

The differential operator for the I-model in the large Kähler structure variable is

$$
\mathcal{L}=\theta^{4}-64 z(8 \theta+7)(8 \theta+5)(8 \theta+3)(8 \theta+1)
$$


Let us then transform to the new variables $(a, b)$

$$
a^{8}=\frac{a_{2} a_{3} a_{4} a_{5}^{4} a_{7}}{a_{0}^{8}}=z_{1} z_{2}^{4}=z, \quad b^{8}=\frac{a_{2} a_{3} a_{4} a_{6}^{8}}{a_{5}^{4} a_{7}}=\frac{z_{1}}{z_{2}^{4}}
$$

in which the limit $b \rightarrow 0$ corresponds to restricting the Kähler moduli space of the II-model to the Kähler moduli space of the I-model. The general discriminants of I and II read in this parametrisation

$$
\begin{aligned}
\Delta_{0(1,1)}^{I I}= & a^{3}\left(1-2^{16} a^{8}\right)+a^{2} b\left(3-7 \cdot 2^{15} a^{8}\right)+a b^{2}\left(3-7 \cdot 41 \cdot 2^{10} a^{8}\right)+b^{3}\left(1-5 \cdot 7 \cdot 2^{9} a^{8}\right) \\
& +a^{4} b^{4}\left(7 \cdot 23 a^{2} b-7 \cdot 3^{2} a b^{2}-2 \cdot 3 \cdot 7 \cdot 11 \cdot 67 a^{3}+3^{3} b^{3}+7^{7} a^{7} b^{4}\right) \\
\Delta_{0,(1,1)}^{I}= & \lim _{b \rightarrow 0} v \Delta_{0(1,1)}^{I I}=a^{3}\left(1-2^{16} a^{8}\right) .
\end{aligned}
$$

The (unnormalized) Yukawa couplings of the II-model are

$$
\begin{aligned}
& K_{111}=\frac{1}{a^{3} \Delta_{0(1,1)}^{I I}}\left(2^{6} a^{2}\left(2^{4} a+3^{2} \cdot 5 b\right)+7^{4} a^{4} b^{5}\left(2^{6} a^{2}-2^{2} 5 a b+3^{2} b^{2}\right)+b^{2}\left(2^{2} 661 a+19 \cdot 41 b\right)\right) \\
& K_{112}=\frac{1}{a^{2} \Delta_{0(1,1)}^{I I}}\left(2^{6} a^{2}+2^{2} 37 a b+3 \cdot 31 b^{2}+7^{3} a^{4} b^{3}\left(2^{10} a^{3}+2^{6} 3 a^{2} b-2^{2} 11 a b^{2}+3^{2} b^{3}\right)\right) \\
& K_{122}=\frac{1}{a \Delta_{0(1,1)}^{I I}}\left(\left(2^{2} a-5 b\right)+7^{2} a^{4} b\left(2^{14} a^{4}+2^{10} 11 a^{3} b+2^{7} 3 a^{2} b^{2}+3^{2} b^{4}\right)\right) \\
& K_{222}=\frac{7 a}{b \Delta_{0(1,1)}^{I I}}\left(2^{2}\left(1-2^{16} a^{8}\right)+a^{3} b\left(2^{1} 4 a^{4}+20^{10} 3 \cdot 29 a^{3} b-2^{7} 5 a^{2} b^{2}-2^{2} 23 a b^{3}+3^{2} b^{4}\right)\right) .
\end{aligned}
$$

We note that as $b \rightarrow 0$, both $K_{112}$ and $K_{122}$ stay finite (and in particular non-zero) while $K_{222}$ blows up as $1 / b$. Comparison with [59] would indicate that $b=0$ corresponds to a conifold point; this will indeed be shown below. Also, $\lim _{b \rightarrow 0} K_{111}=2^{10} / \Delta_{0(1,1)}^{I}$ which agrees with the result obtained by a direct computation on the I model [20].

Let us comment further on the limit $b \rightarrow 0$ of the II model and the correspondence with model I. From table 5.3 we read off that the II model has homogeneous coordinates $\left(y_{2}, y_{3}, \ldots, y_{7}\right)$ which are identified by two $\mathbb{C}^{*}$ actions with exponents $(1,1,1,0,4,-3)$ and $(0,0,0,1,-1,1)$, corresponding to the generators of the Mori cone, $l^{(1)}=(-4,1,1,1,0,4,-3)$ and $l^{(2)}=(-1,0,0,0,1,-1,1)$. The values of the $y_{i}$ are restricted so that we cannot have $y_{2}=y_{3}=y_{4}=y_{5}=0$ or $y_{5}=y_{7}=0$ (the combinatorics leading to this conclusion is identical to that which leads to the calculation of the numerators of the entries for $z_{1}$ and $z_{2}$ in table 5.3). Consider the vector function

$$
\left(y_{2}, \ldots, y_{7}\right) \mapsto\left(y_{2}, y_{3}, y_{4}, y_{6} y_{7}, y_{5} y_{6}\right)
$$


Consideration of the $\mathbb{C}^{*}$ actions shows that this gives a well defined map from the II model to $\mathbf{P}(1,1,1,1,4)$, which blows down the divisor $y_{6}=0$ to a codimension 2 subset. This map is precisely the one that contracts the second generator of the Mori cone, as indicated by the -1 in the corresponding coordinate of $l^{(2)}$. The hyperplane class of $\mathbf{P}(1,1,1,1,4)$ pulls back to $J_{1}$. So we are in a similar situation to that in the first paper of [21], obtaining the relation

$$
\sum_{j} n_{i, j}(I I)=n_{i}(\mathbf{P}(1,1,1,1,4)),
$$

where the spaces in parentheses indicate the space for which the Gromov-Witten invariants are calculated.

It is also interesting to analyze the singularities resulting from the blow down (5.13). In the coordinates $\left(x_{1}, \ldots, x_{5}\right)$ of $\mathbf{P}^{4}(1,1,1,1,4)$, the form of our map shows that the image of the II model is a degree 8 hypersurface containing the surface $x_{4}=x_{5}=0$. The situation is now entirely analogous to that studied by Greene, Morrison and Strominger [60]. The octic equation has the form $x_{4} f+x_{5} g=0$, where $f$ and $g$ have degrees 4 and 7 . This Calabi-Yau is singular at the 28 points $x_{4}=x_{5}=f=g=0$, having conifold singularities (nodes). There are correspondingly 28 vanishing cycles on a nearby smooth octic hypersurface, which, when compactifying the type IIB string on the Calabi-Yau in question, give rise to 28 hypermultiplets which become light as we approach our hypersurface. The 28 vanishing cycles sum to zero, as they are bounded by the complement of 28 balls in the surface $x_{4}=x_{5}=0$. By the argument in [60], we check that this is consistent with $h^{2,1}$ decreasing by 27 from 149 to 122 , while $h^{1,1}$ increases by 1 from 1 to 2 as we perform this conifold transition.

\subsection{Construction of algebraic realizations of the deformation ring}

It is a well-known fact that in certain situations not all of the Kählermoduli space can be described by toric divisors of $X$. Similarly, the deformation of the defining equation of a hypersurface $X^{*}$ in a toric variety $\mathbf{P}_{\Delta^{*}}^{4}$ is in general not sufficient to describe the complex structure moduli space of $X$. More precisely, there are non-toric divisors in $X$ when [26]

$$
\sum_{\text {codimension } \theta^{*}=2, \theta^{*} \in \Delta^{*}} l^{\prime}\left(\theta^{*}\right) l^{\prime}(\theta)
$$

is non-zero. In a similar fashion, when

$$
\sum_{\text {codimension } \theta=2, \theta^{*} \in \Delta} l^{\prime}(\theta) l^{\prime}\left(\theta^{*}\right)
$$

is non-zero there are non-algebraic deformations of the complex structure of $X . l^{\prime}(\theta)$ and $l^{\prime}\left(\theta^{*}\right)$ is the number of interior points in a face $\theta$ and $\theta^{*}$ respectively, where $\theta$ and $\theta^{*}$ are 
dual faces; if $\theta \in \Delta$ is an $n$-dimensional face given by the set of vertices $\left\{\nu^{i_{1}}, \ldots, \nu^{i_{k}}\right\}$ then the dual face, $\theta^{*} \in \Delta^{*}$ is an $3-n$ dimensional face given by,

$$
\theta^{*}=\left\{y \in \Delta^{*} \mid\left(y, \nu^{i_{1}}\right)=\ldots,\left(y \nu^{i_{k}}\right)=-1\right\}
$$

where $\left(\theta^{*}\right)^{*}=\theta^{*}$. We would like to propose a method by which we under certain circumstances can circumvent this problem such that either all of the non-toric divisors in (5.15) or the non-algebraic deformations of the complex structure in (5.16), can be treated by the methods described in section 2 .

Let us assume that there exists a set of points $\left\{\tilde{\nu}^{j_{1}}, \ldots \tilde{\nu}^{j_{r}}\right\}$ in codimension 1 faces of $\Delta$; we will consider the situation in (5.15). In a number of examples which we have studied we have found that it was then possible to promote $\nu^{j_{i}} \in \theta$, given by (5.15), to vertices, while at the same time removing the previous vertices of $\theta$. This also implies that the $\tilde{\nu}^{j_{i}}$ become points in dimension 2 faces. After all this procedure will result in new polyhedra $\tilde{\Delta}^{*}$ and $\tilde{\Delta}$ such that

$$
\tilde{\Delta}^{*} \supset \Delta^{*}, \quad \tilde{\Delta} \subset \Delta .
$$

Hence, as we remove points coming from (5.15) we at the same time increase the contribution from (5.16), i.e. there are more points in codimension 2 faces of $\tilde{\Delta}$ and so the number of non-algebraic deformations of the complex structure has increased. Repeated application of this procedure will then have completely removed the contribution from (5.15). The same can be applied to (5.16) with the obvious changes in the above discussion. Unfortunately, we do not know at this point how general the above idea is.

Let us now consider an example, $X_{12}(1,1,1,3,6)$, in which a non-algebraic sector occurs in the Kähler structure moduli space. Batyrev's original construction leads to the A-representation of the model, where $\Delta_{A}$ is the convex hull of

$$
\begin{array}{ccc}
\nu^{(1)}=(-1,-1,-1,-1)^{0}, & \nu^{(2)}=(-1,-1,-1, \quad 1)^{0}, \quad \nu^{(3)}=(-1,-1, \quad 3,-1)^{0}, \\
\nu^{(4)}=(-1, \quad 11,-1,-1)^{0}, & \nu^{(5)}=(11,-1,-1,-1)^{0} .
\end{array}
$$

The non-algebraic state is related to the fact that the point $\nu^{*(6)}$ in the dual polyhedra $\Delta_{A}^{*}$

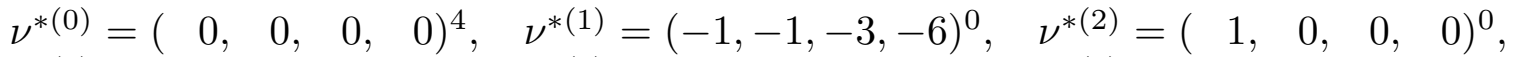

$$
\begin{aligned}
& \nu^{*(3)}=\left(\begin{array}{llll}
0, & 1, & 0, & 0
\end{array}\right)^{0}, \quad \nu^{*(4)}=\left(\begin{array}{llll}
0, & 0, & 1, & 0
\end{array}\right)^{0}, \quad \nu^{*(7)}=\left(\begin{array}{llll}
0, & 0, & 0, & 1
\end{array}\right)^{0},, \\
& \nu^{*(6)}=(0, \quad 0,-1,-2)^{2}, \quad \nu^{*(11)}=\left(\begin{array}{lll}
0, & 0, & 0,-1
\end{array}\right)^{3}
\end{aligned}
$$

lies on a face $\operatorname{conv}\left(\nu^{*(1)}, \nu^{*(2)}, \nu^{*(3)}\right)$, whose dual $\operatorname{conv}\left(\nu^{(2)}, \nu^{(3)}\right)$ is not empty, but contains the point $\tilde{\nu}^{(3)}=(-1,-1,1,0)$. The dimension of the cohomologies are $h^{2,1}=165(0)$ and $h^{1,1}=3(1)$, i.e. in the A-representation, we have an algebraic description of $\mathcal{M}_{2,1}$ but not of $\mathcal{M}_{1,1}$. We hence cut $\Delta_{A}$ in a way, which turns $\nu^{(3)}$ into a corner, i.e. by replacing $\nu^{(3)}$ 
with $\tilde{\nu}^{(3)}$. This leads to the B-representation of the model in which $\Delta_{B}$ is the convex hull of

$$
\begin{aligned}
& \nu^{(1)}=(-1,-1,-1,-1)^{0}, \quad \nu^{(2)}=(-1,-1,-1,1)^{0}, \\
& \nu^{(3)}=(-1,-1, \quad 1,0)^{0}, \quad \nu^{(4)}=(-1, \quad 11,-1,-1)^{0}, \quad \nu^{(5)}=(\quad 11,-1,-1,-1)^{0} .
\end{aligned}
$$

and the enlarged $\Delta_{B}^{*}$ contains

$$
\begin{aligned}
& \nu^{*(0)}=\left(\begin{array}{llll}
0, & 0, & 0, & 0
\end{array}\right)^{4}, \quad \nu^{*(1)}=(-1,-1,-3,-6)^{0}, \quad \nu^{*(2)}=\left(\begin{array}{cccc}
1, & 0, & 0, & 0
\end{array}\right), \\
& \nu^{*(3)}=\left(\begin{array}{llll}
0, & 1, & 0, & 0
\end{array}\right)^{0}, \quad \nu^{*(4)}=\left(\begin{array}{llll}
0, & 0, & 1, & 0
\end{array}\right)^{0}, \quad \nu^{*(5)}=\left(\begin{array}{lll}
0, & 0,-1,2 & 0
\end{array}\right. \\
& \nu^{*(6)}=(0,0,-1,-2)^{2}, \quad \nu^{*(7)}=\left(\begin{array}{ccc}
0, & 0, & 0,1
\end{array}\right)^{1}, \quad \nu^{*(8)}=(0,0,-1,-1)^{3} \text {, }
\end{aligned}
$$

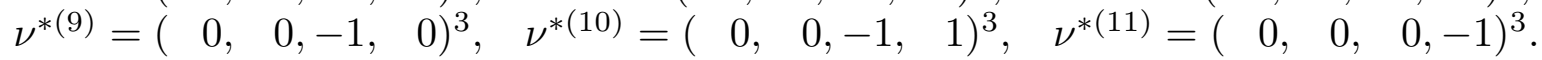

In the B-representation the data of the cohomologies for the new pair $\left(\Delta_{B}, \Delta_{B}^{*}\right)$ are $h^{2,1}=$ $165(55)$ and $h^{1,1}=3(0)$, i.e. we can pick the Laurent monomials of $\nu^{*(0)}, \nu^{*(6)}, \nu^{*(7)}$ as representatives of the ring of Kähler structure deformations. To summarize we can describe the complete complex structure deformations algebraically in the A-representation and the full Kähler structure deformations in the B-representation. We will use the Brepresentation to solve the Kähler deformations part of the $X_{12}(1,1,1,3,6)$ model including the "twisted" sector and investigate how the "untwisted" deformation space of the Arepresentation 22] is embedded in the full deformation space. The generators of the Mori cone for $(5.22)$ are

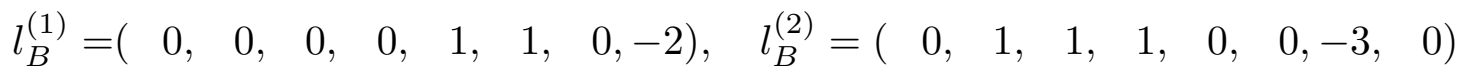

$$
\begin{aligned}
& l_{B}^{(3)}=(-4, \quad 0, \quad 0, \quad 0, \quad 0,-1, \quad 1,4) \text {. }
\end{aligned}
$$

The subspace of the Mori cone, which corresponds to the A-representation, is simply obtained by picking the smallest positive linear combination of the generators $l_{B}^{(i)}$, s.t. the components of the new point $\nu^{*(5)} \in \Delta_{B}^{*}$ are zero, i.e.

$$
\begin{aligned}
& l_{A}^{(1)}=l_{B}^{(1)}+l_{B}^{(3)}=(-4, \quad 0, \quad 0, \quad 0, \quad 1, \quad 0, \quad 1, \quad 2), \\
& l_{A}^{(2)}=(0, \quad 1, \quad 1, \quad 1, \quad 0,0,-3,0) \text {. }
\end{aligned}
$$

The principal parts of the complete set of the Picard-Fuchs equations, which governs the B-representation read

$$
\begin{array}{ll}
\mathcal{L}_{1}=\theta_{1}\left(\theta_{1}-\theta_{3}\right)+\mathcal{O}(z), & \mathcal{L}_{2}=\left(\theta_{3}-3 \theta_{2}\right)\left(\theta_{1}-\theta_{3}\right)+\mathcal{O}(z), \\
\mathcal{L}_{3}=\theta_{3}\left(\theta_{3}-3 \theta_{2}\right)+\mathcal{O}(z), & \mathcal{L}_{4}=\theta_{2}^{3}+\mathcal{O}(z), \\
\mathcal{L}_{5}=\theta_{2}^{2}\left(2 \theta_{2}-\theta_{1}\right)+\mathcal{O}(z) . &
\end{array}
$$


Using the normalization from (5.4) we have the following intersection numbers

$$
\begin{array}{llll}
K_{J_{1}, J_{1}, J_{1}}=18, & K_{J_{1}, J_{1}, J_{2}}=6, & K_{J_{1}, J_{1}, J_{8}}=18, & K_{J_{1}, J_{2}, J_{2}}=2, \\
K_{J_{1}, J_{3}, J_{3}}=18, & K_{J_{1}, J_{2}, J_{3}}=6, & K_{J_{2}, J_{2}, J_{3}}=1, & K_{J_{2}, J_{3}, J_{3}}=3, \\
K_{J_{3}, J_{3}, J_{3}}=9, & \int_{X} c_{2} J_{1}=96, & \int_{X} c_{2} J_{2}=36, & \int_{X} c_{2} J_{3}=102 .
\end{array}
$$

Comparison with the untwisted sector of the A-representation for which one has

$$
K_{I_{1}, I_{1}, I_{1}}=18, \quad K_{I_{1}, I_{1}, I_{2}}=6, \quad K_{I_{1}, I_{2}, I_{2}}=2, \quad \int_{X} c_{2} I_{1}=96, \quad \int_{X} c_{2} I_{2}=36
$$

shows that one has to identify $J_{1}$ with $I_{1}$ and $J_{2}$ with $I_{2}$. For the topological invariants we have the following relations

$$
n_{d_{I_{1}}, d_{I_{2}}}=\sum_{d_{J_{3}}} n_{d_{J_{1}}, d_{J_{2}}, d_{J_{3}}}, \quad n_{d_{J_{1}}, d_{J_{2}}, d_{J_{3}}}=n_{d_{J_{1}}, d_{J_{2}},\left(3 d_{J_{2}}+d_{J_{1}}-d_{J_{3}}\right)} .
$$

This is the analog of the situation for the embeddings of the one moduli cases in the two moduli ones, mentioned at the beginning of this section.

\begin{tabular}{|r|rrrrrrrrrr|r|}
\hline$(i, j)$ & $n_{i j 0}$ & $n_{i j 1}$ & $n_{i j 2}$ & $n_{i j 3}$ & $n_{i j 4}$ & $n_{i j 5}$ & $n_{i j 6}$ & $n_{i j 7}$ & $n_{i j 8}$ & $n_{i j 9}$ & $n_{i j}$ \\
\hline$(0,1)$ & 3 & 0 & 0 & 3 & 0 & 0 & 0 & 0 & 0 & 0 & 6 \\
$(0,2)$ & -6 & 0 & 0 & 0 & 0 & 0 & -6 & 0 & 0 & 0 & -12 \\
$(0,3)$ & 27 & 0 & 0 & 0 & 0 & 0 & 0 & 0 & 0 & 27 & 54 \\
\hline$(1,0)$ & 108 & 108 & 0 & 0 & 0 & 0 & 0 & 0 & 0 & 0 & 216 \\
$(2,0)$ & 0 & 324 & 0 & 0 & 0 & 0 & 0 & 0 & 0 & 0 & 324 \\
$(3,0)$ & 0 & 108 & 108 & 0 & 0 & 0 & 0 & 0 & 0 & 0 & 216 \\
$(4,0)$ & 0 & 0 & 324 & 0 & 0 & 0 & 0 & 0 & 0 & 0 & 324 \\
$(5,0)$ & 0 & 0 & 108 & 108 & 0 & 0 & 0 & 0 & 0 & 0 & 216 \\
\hline$(1,1)$ & 0 & -216 & 0 & -216 & 0 & 0 & 0 & 0 & 0 & 0 & -432 \\
$(1,2)$ & 0 & 540 & 0 & 0 & 0 & 0 & 540 & 0 & 0 & 0 & 1080 \\
$(1,3)$ & 0 & -3456 & 0 & 0 & 0 & 0 & 0 & 0 & 0 & -3456 & -6912 \\
\hline$(2,1)$ & 0 & -648 & 5778 & 5778 & -648 & 0 & 0 & 0 & 0 & 0 & 10260 \\
$(2,2)$ & 0 & 1620 & -23112 & 0 & 1296 & 0 & -23112 & 1620 & 0 & 0 & -41688 \\
$(2,3)$ & 0 & -10368 & 202986 & 0 & -3240 & 0 & 0 & -3240 & 0 & 202986 & 378756 \\
\hline
\end{tabular}

Table 5.4 The invariants of rational curves of degree $(i, j, k)$ for $X_{12}(1,1,1,3,6)$ in the Brepresentation including the "twisted state". The last column contains for comparison the invariants which correspond to the "untwisted sector" in the A-representation. 
Finally, it is very instructive to investigate the intersection numbers in the basis, which is suggested by the Laurent-monomials divisor relation in the A-representation. Denoting this divisors with the same labels as the corresponding points in $\Delta_{A}^{*}$ we have after the identification

$$
I_{0}=I_{1}, \quad I_{6}=I_{1}-3 I_{2}
$$

the from $(5.27)$ the following topological data

$$
K_{I_{0}, I_{0}, I_{0}}=18, \quad K_{I_{6}, I_{6}, I_{6}}=18, \quad \int_{X} c_{2} I_{0}=96, \quad \int_{X} c_{2} I_{6}=-12 .
$$

The divisor $I_{6}$ with multiplicity two on the triangle $\operatorname{conv}\left(\nu^{*(1)}, \nu^{*(2)}, \nu^{*(3)}\right)$ has to be split symmetrically between $I_{6}^{\prime}$ and $I_{6}^{\prime \prime}$. In this way we arrive at the intersection numbers [22]

$$
K_{I_{0}, I_{0}, I_{0}}=18, K_{I_{6}^{\prime}, I_{6}^{\prime}, I_{6}^{\prime}}=9, K_{I_{6}^{\prime \prime}, I_{6}^{\prime \prime}, I_{6}^{\prime \prime}}=9, \int_{X} c_{2} I_{0}=96, \int_{X} c_{2} I_{6}^{\prime}=-6, \int_{X} c_{2} I_{6}^{\prime \prime}=-6 .
$$

Now in order to make the consistency check, that the A- and the B-representation are homotopy equivalent we must find linear transformation, by which (5.26) transform into (5.31). As the reader may check the simple transformation

$$
I_{0}=J_{1}, \quad I_{6}^{\prime}=J_{3}-3 J_{2}, \quad I_{6}^{\prime \prime}=J_{1}-J_{3}
$$

has precisely this property. Note that this is also in agreement with the second relation in $(5.28)$.

We will now mention an example for the situation when there are non-algebraic complex structure deformations. This also serves as an important technical application of the discussion in the previous section. Let us use the $\left(\Delta_{I I}, \Delta_{I I}^{*}\right)$ - and the $\left(\Delta_{I I I}, \Delta_{I I I}^{*}\right)$ representation of the moduli space $\mathcal{M}_{2,1}\left(X_{14}(1,2,2,2,7)\right)$ as representations in which all deformations are algebraic, see section 5.2. The possibility of utilizing $X_{7}(1,1,1,1,3)$ in this way was already pointed out, however in general we need non-Landau-Ginzburg models, as the second one, to obtain an algebraic representation. We can understand the modification of $\Delta_{I V}^{*}$ as follows. The point $\nu^{*(6)}$ on the edge $\operatorname{conv}\left(\nu^{*(1)}, \nu^{*(5)}\right)$ gives rise to the non-algebraic states of the deformation space for $\mathcal{M}_{2,1}$ in the representation $\left(\Delta_{I V}, \Delta_{I V}^{*}\right)$, because its dual face $\operatorname{conv}\left(\nu^{(6)}, \nu^{(7)}, \nu^{(8)}\right)$ contains 15 inner points. Removing the points $\nu^{*(1)}, \nu^{*(8)}\left(\nu^{*(1)}, \nu^{*(7)}\right)$ promotes the point $\nu^{*(6)}$ into an edge. The polyhedra $\Delta_{I I}\left(\Delta_{I I I}\right)$ contains $15(18)$ points more then $\Delta_{I V}(3(3)$ on codim $4,9(3)$ on codim $3,3(9)$ on codim 2 and $0(3)$ on codim 1 faces), which correspond (partly) to the missing algebraic deformations.

Finally, let us show, in cases where there are no points in codimension 1 faces, how we can circumvent the failure of removing the non-algebraic complex structure deformation. 
Consider $X_{8}(1,1,2,2,2)^{2,86}$. Beside the five corners the simplicial polyhedron $\Delta^{*}$ contains the interior point $\nu_{0}^{*}=(0,0,0,0)$ as well as one other point $\nu_{6}^{*}(0,-1,-1,-1)$ on a codimension three edge $\theta^{*}$ spanned by $\nu_{1}^{*}=(1,0,0,0)$ and $\nu_{5}=(-1,-2,-2,-2)$. The dual face $\theta$ of codimension two in $\Delta$ is spanned by $(3,-1,-1,-1),(-1,3,-1,-1),(-1,-1,3,-1)$ and contains three points $(1,0,0,-1),(0,1,0,-1),(0,0,1,-1)$. By (5.15) we hence count three non-algebraic $(1,2)$-forms.

As there are no points on codimension one faces in $\Delta^{*}$, cutting one of the corners $\nu_{1}^{*}$ or $\nu_{5}^{*}$ will not lead to a reflexive pair $\left(\Delta_{B}, \Delta_{B}^{*}\right)$, which could serve as an algebraic description for the complex structure deformations. Instead use $|H|=\left(x_{1}^{2}, x_{1} x_{2}, x_{2}^{2}, x_{3}, x_{4}, x_{5}\right)$ to map to $\mathbf{P}^{5}$; the image is contained in the singular quadric $y_{1} y_{3}=y_{2}^{2}$. The singularity $\left(y_{1}=y_{2}=y_{3}=0\right)$ gets blown up to produce the extra exceptional toric divisor. The degree 8 equation becomes degree 4 in the $y_{i}$ 's. So we get a degenerate blown up $\mathbf{P}^{5}[2,4]$.

Now vary the quadric from rank 3 to rank 4 (the prototype, which is unique up to coordinate change, is $\left.y_{1} y_{2}=y_{3} y_{4}\right)$. The quadric is singular at the smaller locus $y_{1}=y_{2}=$ $y_{3}=y_{4}=0$, so can still be blown up to produce the extra $(1,1)$-form. But the space of rank 3 quadrics has codimension 3 inside the space of rank 4 quadrics — so by allowing rank 4 quadrics, we have introduced 3 more parameters.

\section{Discussion}

The purpose of this article has been two-fold. On one hand we have shown that the original ideas of Candelas et al [18], studying the moduli space of complex structure and Kahler structure deformations by means of special geometry and mirror symmetry extends to general Calabi-Yau hypersurfaces in toric varieties. Although we do not claim to have shown this for all Calabi-Yau manifolds of this type we believe that the non-trivial examples considered is evidence that a description along the lines outlined below will serve the purpose.

Starting from $X$, its mirror, $X^{*}$ is constructed via the dual polyhedron. In resolving the singularities, subdividing $\Delta^{*}$, one has in general several subdivisions which lend themselves to a Kähler resolution, corresponding to a different geometric interpretation in the phase picture. For each of these subdivisions, we construct the generators of the Mori cone for $P_{\Delta^{*}}$. Note that in general it is not true that the Mori cones of the toric variety and $X$ are the same. Using linear relations between the generators we find all second and third order differential operators. Application of INSTANTON [46] gives information on whether this is a sufficient set of operators. In general that is not the case.

The next step is to find the Batyrev-Cox ring and then restrict to a subset of the coordinates, for which the original polyhedron $\Delta$ is unresolved. The explicit use of the monomial representation, and in particular the ideal allows us to compute all the second 
order relations. Although in the example studied in section 3 this turned out to be sufficient, we believe that it will not be so for the general situation. Rather, we need to consider order three monomials and then reapply the reduction method. Collecting all of the order two and order three operators constructed in this way, one will be able to first show that the classical intersection numbers agree with the above computation. Finally, the instanton contributions can (in principle) be computed to arbitrary multidegree of the rational curve.

There is an alternative approach to the method described here which is under investigation. It appears that the principal parts of the Picard-Fuchs operators can be deduced from the topological couplings directly. This would be a consequence of mirror symmetry if mirror symmetry could be established for Batyrev's construction via dual polyhedra. It is more likely that the result about the principal parts can be proven directly within a purely mathematical framework. It can be checked that the principal parts of all the second and third order operators in this paper may be derived much more easily by the method under investigation. At present however, this method is less powerful since its correctness rests on the validity of at least one conjecture.

Using these methods we have presented some new ways of exploring the moduli space. Given a toric variety represented in terms of a reflexive polyhedron, $\Delta$, we may alter $\Delta$ with the requirement that the new polytope, $\tilde{\Delta}$ is reflexive. In such a fashion new theories can be constructed as hypersurfaces in toric varieties based on $\tilde{\Delta}$, taking a first step towards a classification of all reflexive polyhedra, and hence further enlarge the class of $N=2 \mathrm{SCFT}$. (Recall that the toric description is equivalent to the $N=2$ supersymmetric gauged linear sigma model [51,41], where the latter is believed to have non-trivial fixed point in the IR-limit.) A less severe alteration of $\Delta$ such that the Hodge numbers do not change for the hypersurfaces in question can still result in interesting phenomena. In some cases it is possible to find a $\tilde{\Delta}\left(\tilde{\Delta}^{*}\right)$ such that all the Kähler (complex) structure deformations can be described algebraically. Thus, we can study the moduli space for all deformations including the so called twisted deformations. At this point it is not clear how general this phenomenon is; we know of twisted complex structure deformations which seems to allude the above prescription. Finally, given a $\Delta$ we can choose to embed the hypersurface in a variety of ways, and in particular restricting to (singular) hypersurfaces which however in their own right correspond to smooth (possibly after some desingularization) Calabi-Yau manifolds. It may be that it is possible to understand all hypersurfaces in toric varieties as special points in a moduli space. Naively, although the process in going from one theory to another is singular this may provide us with a better understanding of the theories at hand. However, in light of the recent developments in understanding conifold transitions in type IIB string theories in terms of condensation of black holes 61,60; though, rather than being singular the transition is smooth when the effect of the massless black holes 
are properly taken into account. Indeed, we find that the transition between model I and II in section 5.2 can be explained in perfect analogy with [60].

Related to this is the recent evidence of a spacetime duality between type II string theory compactified on Calabi-Yau manifolds and that of compactification of the heterotic string on $K 3 \times T^{2}[62$. It would be interesting to see if it is possible to find the heterotic dual corresponding to the type of Calabi-Yau compactifications discussed in this paper.

On the other hand, with the improved knowledge of the moduli space of heterotic string vacua one could try to make some first modest steps in probing the phenomenological implications. Apart from the well-known Yukawa couplings the moduli dependence of the threshold corrections to the gauge couplings in string theory (as well as in the effective quantum field theory) [9] have come to be understood. We have computed the corrections for the two parameter models studied in this article (as was done for some Fermat-type models in [21,22]), and since the techniques at hand allows us to find the Picard-Fuchs equation (and its solutions) for any model, similarly the threshold corrections can be computed for any Calabi-Yau hypersurface for which our method applies. Finally, it is interesting to note that so many quantities only depend on the massless part of the theory, or can be deduced from the massless sector. One may wonder what other non-trivial properties can be computed in a similar fashion.

Acknowledgments: It is a pleasure to thank D.R. Morrison and M.R. Plesser for useful discussions. P.B. was supported by the DOE grant DE-FG02-90ER40542 and acknowledges the hospitality of the Theory Division, CERN and Aspen Center for Physics where this work was initiated. S.K. was supported by NSF grant DMS-9311386. A.K. thanks Maximilian Kreuzer, Wolfgang Lerche, Bong Lian, Stefan Theisen, Shing-Tung Yau and especially Shinobu Hosono for discussions and comments. 


\section{Appendix A. Further examples}

A.1. The case $X_{8}(1,1,1,2,3)_{-208}^{2,106}$

$$
\begin{aligned}
& l^{(1)}=(-2,0,0,0,0,1,1), \quad l^{(2)}=(-2,1,1,1,2,0,-3) \\
& \mathcal{L}_{1}=\theta_{1}\left(\theta_{1}-3 \theta_{2}\right)-\left(2 \theta_{1}+\theta_{2}\right)\left(2 \theta_{1}+\theta_{2}-1\right) z_{1} \\
& \mathcal{L}_{2}=\left(1-4 z_{1}\right)^{2}\left[\theta_{2}^{2}\left(\theta_{1}-4 \theta_{2}\right)-2 z_{1} \theta_{2}^{2}\left(2 \theta_{1}+2 \theta_{2}+1\right)+4 z_{2} \prod_{i=0}^{2}\left(\theta_{1}-3 \theta_{2}-i\right)\right] \\
& +16\left(1-4 z_{1}\right) z_{1} z_{2}\left(\theta_{1}-3 \theta_{2}\right)\left(\theta_{1}-3 \theta_{2}-1\right)\left(\left(\theta_{1}-3 \theta_{2}-2\right)+2\left(2 \theta_{1}+2 \theta_{2}+1\right)\right) \\
& +128 z_{1}^{2} z_{2}\left(\theta_{1}-3 \theta_{2}\right)\left(2 \theta_{1}+2 \theta_{2}+1\right)+16 z_{1} z_{2}\left(\theta_{1}-3 \theta_{2}\right)\left(\theta_{1}-3 \theta_{2}-1\right) \\
& K_{111}=4 \frac{\left(1-4 z_{1}\right)\left(9+64 z_{1}+96 z_{1}^{2}+16 z_{1}^{3}\right)+\left(243+432 z_{1}+4608 z_{1}^{2}+4096 z_{1}^{3}\right) z_{2}}{z_{1}^{3} \Delta} \\
& K_{112}=4 \frac{\left(1-4 z_{1}\right)^{2}\left(3+12 z_{1}+2 z_{1}^{2}\right)-\left(81-432 z_{1}-512 z_{1}^{2}+4096 z_{1}^{3}\right) z_{2}}{z_{1}^{2} z_{2} \Delta} \\
& K_{122}=4 \frac{\left(1-4 z_{1}\right)^{3}\left(1+z_{1}\right)+\left(27-336 z_{1}+1536 z_{1}^{2}-4096 z_{1}^{3}\right) z_{2}}{z_{1} z_{2}^{2} \Delta} \\
& K_{222}=\frac{\left(1-4 z_{1}\right)^{4}+4\left(9-176 z_{1}+1536 z_{1}^{2}+4096 z_{1}^{3}\right) z_{2}}{z_{2}^{3} \Delta}, \\
& \Delta=\left(1-4 z_{1}\right)^{5}+z_{2}\left(27-576 z_{1}+5120 z_{1}^{2}-180224 z_{1}^{3}-131072 z_{1}^{4}-4194304 z_{1}^{3} z_{2}\right) \\
& K_{J_{1} J_{1} J_{1}}=36, K_{J_{1} J_{1} J_{2}}=12, K_{J_{1} J_{2} J_{2}}=4, K_{J_{2} J_{2} J_{2}}=1 \\
& \int c_{2} J_{1}=96, \quad \int c_{2} J_{2}=34
\end{aligned}
$$

$r_{0}=-\frac{1}{6}, s_{1}=-9, s_{2}=-\frac{23}{6}, n_{8 i-j, i}=n_{j, i}$

\begin{tabular}{|l|rrrrrrr|}
\hline$n_{i, j}$ & $j=0$ & $j=1$ & $j=2$ & $j=3$ & $j=4$ & $j=5$ & $j=6$ \\
\hline$n_{j, 0}^{r}$ & 0 & 40 & 0 & $\forall j>1$ & & & \\
$n_{j, 0}^{e}$ & 0 & $\forall j$ & & & & & \\
\hline$n_{j, 1}^{r}$ & 3 & -80 & 780 & 54192 & 121410 & 54192 & 780 \\
$n_{j, 1}^{e}$ & 0 & 0 & 0 & 40 & 200 & 40 & 0 \\
\hline$n_{j, 2}^{r}$ & -6 & 200 & -3120 & 29640 & -425600 & 5297640 & 558340176 \\
$n_{j, 2}^{e}$ & 0 & 0 & 0 & 0 & -400 & 62032 & 6426648 \\
\hline$n_{j, 3}^{r}$ & 27 & -1280 & 27580 & -365040 & 3953900 & -41185408 & 371614680 \\
$n_{j, 3}^{e}$ & -10 & 360 & -6240 & 69160 & -547340 & 3041832 & -8415720 \\
\hline
\end{tabular}

Table A.1 The invariants of rational and elliptic curves of degree $(i, j), n_{i, j}^{r}$ and $n_{i, j}^{e}$ respectively, for $X_{8}(1,1,1,2,3)$. 
$J_{1}$ is the class of cubic polynomials; $J_{2}$ is the class of linear polynomials. The exceptional divisor $E$ is $\mathbf{P}^{2}$, and $J_{1}=3 J_{2}+E$. From this, we see that the numbers $n_{0, j}$ are identical to those for $\mathbf{P}^{4}(1,1,1,1,3)$.

A.2. The case $X_{9}(1,1,2,2,3)_{-168}^{2,86}$

$$
\begin{gathered}
l^{(1)}=(-3,-1,-1,1,1,0,3), \quad l^{(2)}=(-1,1,1,0,0,1,-2) \\
\mathcal{L}_{1}=\theta_{2}\left(\theta_{2}-\theta_{1}\right)^{2}-\left(3 \theta_{1}+\theta_{2}\right)\left(3 \theta_{1}-2 \theta_{2}+2\right)\left(3 \theta_{1}-2 \theta_{2}+1\right) z_{2} \\
\mathcal{L}_{2}=\left(\theta_{2}-\theta_{1}\right)^{2}-\left(\theta_{2}-\theta_{1}\right)\left(3 \theta_{1}-\theta_{2} \theta_{2}\right)+4 \theta_{1}\left(3 \theta_{1}-2 \theta_{2}\right)+3 z_{1}\left(3 \theta_{1}-2 \theta_{2}\right)\left(3 \theta_{1}-2 \theta_{2}-1\right) \\
-48 z_{1} z_{2}\left(3 \theta_{1}+\theta_{2}+1\right)\left(3 \theta_{1}+\theta_{2}+2\right)-48 z_{1} z_{2}\left(3 \theta_{1}+\theta_{2}+1\right)\left(3 \theta_{1}-2 \theta_{2}\right)-16 z_{1}\left(\theta_{2}-\theta_{1}\right)^{2} \\
K_{111}=\frac{1}{z_{1}^{3} \Delta}\left(6-5 z_{1}-48 z_{2}+269 z_{1} z_{2}-768 z_{1}^{2} z_{2}+96 z_{2}^{2}-1677 z_{1} z_{2}^{2}\right. \\
\left.\quad+6144 z_{1}^{2} z_{2}^{2}+2916 z_{1} z_{2}^{3}-11664 z_{1}^{2} z_{2}^{3}\right) \\
K_{112}=\frac{1}{z_{1}^{2} z_{2} \Delta}\left(9-9 z_{1}-72 z_{2}-400 z_{1} z_{2}+256 z_{1}^{2} z_{2}+144 z_{2}^{2}+4005 z_{1} z_{2}^{2}-\right. \\
\left.\quad 11520 z_{1}^{2} z_{2}^{2}-8748 z_{1} z_{2}^{3}+34992 z_{1}^{2} z_{2}^{3}\right) \\
K_{122}=\frac{1}{z_{1} z_{2}^{2} \Delta}\left(13-13 z_{1}-106 z_{2}-1525 z_{1} z_{2}+1280 z_{1}^{2} z_{2}+216 z_{2}^{2}+14634 z_{1} z_{2}^{2}+\right. \\
\left.\quad 13824 z_{1}^{2} z_{2}^{2}-32805 z_{1} z_{2}^{3}-104976 z_{1}^{2} z_{2}^{3}\right) \\
K_{222}=\frac{1}{z_{2}^{3} \Delta}\left(17-17 z_{1}-153 z_{2}-2610 z_{1} z_{2}+2304 z_{1}^{2} z_{2}+324 z_{2}^{2}+\right. \\
\left.\quad 32886 z_{1} z_{2}^{2}+20736 z_{1}^{2} z_{2}^{2}-78732 z_{1} z_{2}^{3}+314928 z_{1}^{2} z_{2}^{3}\right) \\
K_{J_{1} J_{1} J_{1}=}=-\frac{1}{6}, s_{1}=-5, s_{J_{1} J_{1} J_{2}=9, K_{J_{1} J_{2} J_{2}}=13, K_{J_{2} J_{2} J_{2}}=17, \int c_{2} J_{1}=48}\left(c_{2} J_{2}=74\right. \\
\Delta=1-z_{1}-8 z_{2}-531 z_{1} z_{2}+512 z_{1}^{2} z_{2}+16 z_{2}^{2}+4335 z_{1} z_{2}^{2}+74496 z_{1}^{2} z_{2}^{2}- \\
65536 z_{1}^{3} z_{2}^{2}-8748 z_{1} z_{2}^{3}-1539648 z_{1}^{2} z_{2}^{3}+8503056 z_{1}^{2} z_{2}^{4}-14348907 z_{1}^{2} z_{2}^{5}
\end{gathered}
$$




\begin{tabular}{|l|rrrrrrr|}
\hline$n_{i, j}$ & $j=0$ & $j=1$ & $j=2$ & $j=3$ & $j=4$ & $j=5$ & $j=6$ \\
\hline$n_{0, j}^{r}$ & 0 & -2 & 0 & $\forall j>1$ & & & \\
$n_{0, j}^{e}$ & 0 & $\forall j$ & & & & & \\
\hline$n_{1, j}^{r}$ & 1 & 640 & 641 & 4 & 5 & 7 & 9 \\
$n_{1, j}^{e}$ & 0 & $\forall j$ & & & & & \\
\hline$n_{2, j}^{r}$ & 0 & 0 & 10032 & 208126 & 8734 & -2596 & -3900 \\
$n_{2, j}^{e}$ & 0 & 0 & 0 & 640 & 12 & 24 & 36 \\
\hline$n_{3, j}^{r}$ & 0 & 0 & 0 & 288384 & 23177356 & 23347504 & 798855 \\
$n_{3, j}^{e}$ & 0 & 0 & 0 & -1280 & 158140 & 164290 & 17152 \\
\hline
\end{tabular}

Table A.2 The invariants of rational and elliptic curves of degree $(i, j), n_{i, j}^{r}$ and $n_{i, j}^{e}$ respectively, for $X_{9}(1,1,2,2,3)$.

$J_{1}$ is the class of quadratic polynomials; $J_{2}$ is the class of cubic polynomials. We have $3 J_{1}=2 J_{2}+E$, where the exceptional divisor $E$ is a rational ruled surface, with map to $\mathbf{P}^{1}$ defined by $\left(x_{3}, x_{4}\right)$. This shows as in the case of $\mathbf{P}^{4}(1,1,1,2,2)$ that $n_{0,1}^{r}=-2$. Similarly, $n_{1, j}^{r}=2 j-3$ for $j \geq 4$, corresponding to the sections of the ruled surface.

A.3. The case $X_{14}(1,1,2,3,7)_{-260}^{2,132}$

$$
\begin{gathered}
l^{(1)}=(0,-2,-2,-4,1,0,7), \quad l^{(2)}=(-2,1,1,2,0,1,-3) \\
\mathcal{L}_{1}=\theta_{1}\left(7 \theta_{1}-3 \theta_{2}\right)-4 z_{1} z_{2}^{2}\left(2 \theta_{2}+1\right)\left(2 \theta_{2}+3\right) \\
\mathcal{L}_{2}=2\left(1-64 z_{1} z_{2}^{2}\right)^{2}\left[\left(\theta_{2}-2 \theta_{1}\right)^{3}-z_{2}\left(7 \theta_{1}-3 \theta_{2}\right)\left(7 \theta_{1}-3 \theta_{2}-1\right)\left(7 \theta_{1}-3 \theta_{2}-2\right)\right] \\
-14336 z_{1}^{2} z_{2}^{5}\left(2 \theta_{2}+1\right)\left(7 \theta_{1}-3 \theta_{2}\right)-128 z_{1} z_{2}^{3}\left(7 \theta_{1}-3 \theta_{2}\right)\left(7 \theta_{1}-3 \theta_{2}-1\right) \\
-32 z_{1} z_{2}^{3}\left(1-64 z_{1} z_{2}^{2}\right)\left(7 \theta_{1}-3 \theta_{2}\right)\left(7 \theta_{1}-3 \theta_{2}-1\right)\left[7\left(2 \theta_{2}+1\right)+4\left(7 \theta_{1}-3 \theta_{2}-2\right)\right] \\
K_{111}=\frac{1}{z_{1}^{3} \Delta}\left(9\left(1+27 z_{2}\right)-16 z_{1} z_{2}^{2}\left(11+378 z_{2}\right)-256 z_{1}^{2} z_{2}^{4}\left(88+2205 z_{2}\right)-\right. \\
K_{112}=\frac{1}{z_{1}^{2} z_{2} \Delta}\left(21\left(1+27 z_{2}\right)-336 z_{1} z_{2}^{2}\left(2+63 z_{2}\right)-256 z_{1}^{2} z_{2}^{4}\left(160+3773 z_{2}\right)-131072 z_{1}^{3} z_{2}^{6}\right) \\
K_{122}=\frac{1}{z_{1} z_{2}^{2} \Delta}\left(49\left(1+27 z_{2}\right)-64 z_{1} z_{2}^{2}\left(34+1029 z_{2}\right)-512 z_{1}^{2} z_{2}^{4}\left(120+2401 z_{2}\right)\right) \\
K_{222}=\frac{1}{z_{2}^{3} \Delta}\left(114+1029 z_{2}-80 z_{1} z_{2}^{2}\left(80+2401 z_{2}\right)-573444 z_{1}^{2} z_{2}^{4}\right) \\
\Delta=1+27 z_{2}-16 z_{1} z_{2}^{2}\left(16-441 z_{2}\right)+2048 z_{1}^{2} z_{2}^{4}\left(12+343 z_{2}\right)- \\
4096 z_{1}^{3} z_{2}^{6}\left(256+38416 z_{2}+823543 z_{2}^{2}\right)+16777216 z_{1}^{4} z_{2}^{7}
\end{gathered}
$$




$$
K_{J_{1} J_{1} J_{1}}=9, K_{J_{1} J_{1} J_{2}}=21, K_{J_{1} J_{2} J_{2}}=49, K_{J_{2} J_{2} J_{2}}=114, \int c_{2} J_{1}=66, \int c_{2} J_{2}=156
$$

$r_{0}=-\frac{1}{6}, s_{1}=-\frac{13}{2}, s_{2}=-14$

\begin{tabular}{|l|rrrrr|}
\hline$n_{i, j}$ & $j=0$ & $j=1$ & $j=2$ & $j=3$ & $j=4$ \\
\hline$n_{j, 0}^{r}$ & 0 & $\forall j$ & & & \\
$n_{j, 0}^{e}$ & 0 & $\forall j$ & & & \\
\hline$n_{j, 1}^{r}$ & 3 & 0 & $\forall j>0$ & & \\
$n_{j, 1}^{e}$ & 0 & $\forall j$ & & & \\
\hline$n_{j, 2}^{r}$ & -6 & 220 & 0 & $\forall j>1$ & \\
$n_{j, 2}^{e}$ & 0 & 1 & 0 & $\forall j>1$ & \\
\hline$n_{j, 3}^{r}$ & 27 & -440 & 0 & $\forall j>1$ & \\
$n_{j, 3}^{e}$ & -10 & -2 & 0 & $\forall j>1$ & \\
\hline$n_{j, 4}^{r}$ & -192 & 1100 & 260 & 0 & $\forall j>2$ \\
$n_{j, 4}^{e}$ & 231 & 5 & 2 & 0 & $\forall j>2$ \\
\hline
\end{tabular}

Table A.3 The invariants of rational and elliptic curves of degree $(i, j), n_{i, j}^{r}$ and $n_{i, j}^{e}$ respectively, for $X_{14}(1,1,2,3,7)$.

$J_{1}$ is the class of cubic polynomials and $J_{2}$ is the class of degree seven polynomials. The exceptional divisor $E$ is a $\mathbf{P}^{2}$, and $7 J_{1}=3 J_{2}+E$. From this, we see that the numbers $n_{0, j}$ are identical to those for $\mathbf{P}^{4}(1,1,1,1,3)$.

Note that the Kähler cone of the toric variety differs from the Kähler cone of the Calabi-Yau hypersurface. The Mori cone of $X_{14}(1,1,2,3,7)$ is generated by $l^{(1)}+2 l^{(2)}, l^{(2)}$, i.e. by the curves of type $(1,2)$ and $(0,1)$. A curve of type $(a, b)$ with $b<2 a$ would be contained in the locus $x_{1}=x_{2}=x_{3}=0$, which is a curve on the toric variety but a point on the Calabi-Yau hypersurface. 


\section{Appendix B. Pichard-Fuchs operators, intersection and instanton numbers for} $\mathbf{P}^{4}(1,2,3,3,4)$

$$
\begin{aligned}
& \mathcal{L}_{1}=\left(\theta_{2}+\theta_{4}-\theta_{5}\right)\left(-2 \theta_{1}+\theta_{4}\right)+\mathcal{O}\left(z_{i}\right) \\
& \mathcal{L}_{2}=\left(\theta_{1}-2 \theta_{4}+\theta_{5}\right)\left(-2 \theta_{2}+\theta_{5}\right)+\mathcal{O}\left(z_{i}\right) \\
& \mathcal{L}_{3}=\left(-2 \theta_{1}+\theta_{4}\right)\left(-2 \theta_{2}+\theta_{5}\right)+\mathcal{O}\left(z_{i}\right) \\
& \mathcal{L}_{4}=\left(3 \theta_{2}-2 \theta_{3}\right)\left(-2 \theta_{1}+\theta_{4}\right)+\mathcal{O}\left(z_{i}\right) \\
& \mathcal{L}_{5}=\theta_{1}\left(-2 \theta_{2}+\theta_{5}\right)+\mathcal{O}\left(z_{i}\right) \\
& \mathcal{L}_{6}=\left(\theta_{1}-2 \theta_{4}+\theta_{5}\right)\left(3 \theta_{2}-2 \theta_{3}\right)+\mathcal{O}\left(z_{i}\right) \\
& \mathcal{L}_{7}=\theta_{1}\left(\theta_{1}+\theta_{3}-\theta_{5}\right)\left(\theta_{1}-2 \theta_{4}+\theta_{5}\right)+\mathcal{O}\left(z_{i}\right) \\
& \mathcal{L}_{8}=\left(\theta_{2}+\theta_{4}-\theta_{5}\right)\left(\theta_{1}+\theta_{3}-\theta_{5}\right)\left(3 \theta_{2}-2 \theta_{3}\right)+\mathcal{O}\left(z_{i}\right) \\
& \mathcal{L}_{9}=\left(\theta_{3}-\theta_{2}\right)^{2}\left(\theta_{1}+\theta_{3}-\theta_{5}\right)+\mathcal{O}\left(z_{i}\right) \\
& \mathcal{L}_{10}=\theta_{1}\left(\theta_{2}+\theta_{4}-\theta_{5}\right)\left(\theta_{1}+\theta_{3}-\theta_{5}\right)+\mathcal{O}\left(z_{i}\right) \\
& \mathcal{L}_{11}=\left(-2 \theta_{1}+\theta_{4}\right)\left(-2 \theta_{2}+\theta_{5}\right)\left(-2 \theta_{2}+\theta_{5}-1\right)+\mathcal{O}\left(z_{i}\right) \\
& \mathcal{L}_{12}=\left(\theta_{1}+\theta_{4}-\theta_{5}\right)\left(3 \theta_{2}-2 \theta_{3}\right)\left(-2 \theta_{1}+\theta_{4}\right)+\mathcal{O}\left(z_{i}\right) \\
& \mathcal{L}_{13}=\left(\theta_{1}-2 \theta_{4}+\theta_{5}\right)\left(\theta_{1}-2 \theta_{4}+\theta_{5}-1\right)\left(3 \theta_{2}-2 \theta_{3}\right)+\mathcal{O}\left(z_{i}\right) \\
& \mathcal{L}_{14}=\theta_{1}\left(\theta_{2}+\theta_{4}-\theta_{5}\right)\left(-2 \theta_{2}+\theta_{5}\right)+\mathcal{O}\left(z_{i}\right) \\
& \mathcal{L}_{15}=\theta_{1}\left(-2 \theta_{2}+\theta_{5}\right)\left(-2 \theta_{2}+\theta_{5}-1\right)+\mathcal{O}\left(z_{i}\right) \\
& \mathcal{L}_{16}=\theta_{1}\left(\theta_{1}-2 \theta_{4}+\theta_{5}\right)\left(3 \theta_{2}-2 \theta_{3}\right)+\mathcal{O}\left(z_{i}\right) \\
& \mathcal{L}_{17}=\left(\theta_{3}-\theta_{2}\right)^{2}\left(-2 \theta_{1}+\theta_{4}\right)+\mathcal{O}\left(z_{i}\right) \\
& \mathcal{L}_{18}=\theta_{1}\left(\theta_{2}+\theta_{3}-\theta_{5}\right)\left(3 \theta_{2}-2 \theta_{3}\right)+\mathcal{O}\left(z_{i}\right) \\
& \mathcal{L}_{19}=\theta_{1}\left(3 \theta_{2}-2 \theta_{3}\right)\left(-2 \theta_{2}+\theta_{5}\right)+\mathcal{O}\left(z_{i}\right) \\
& \mathcal{L}_{20}=\left(\theta_{2}+\theta_{4}-\theta_{5}\right)\left(3 \theta_{2}-2 \theta_{3}\right)+\mathcal{O}\left(z_{i}\right) \\
& \mathcal{L}_{21}=\theta_{1}\left(\theta_{1}-2 \theta_{4}+\theta_{5}\right)-4\left(\theta_{3}-\theta_{2}\right)\left(\theta_{1}-2 \theta_{4}+\theta_{5}\right)+\mathcal{O}\left(z_{i}\right) \\
& \mathcal{L}_{22}=-\theta_{1}\left(\theta_{1}+\theta_{3}-\theta_{5}\right)+\theta_{1}\left(-2 \theta_{1}+\theta_{4}\right)-4\left(\theta_{3}-\theta_{2}\right)\left(-2 \theta_{1}+\theta_{4}\right) \\
& +\left(\theta_{3}-\theta_{2}\right)\left(\theta_{1}+\theta_{2}+\theta_{3}-\theta_{5}\right)-\left(\theta_{1}+\theta_{3}-\theta_{5}\right)\left(3 \theta_{2}-2 \theta_{3}\right) \\
& +2\left(3 \theta_{2}-2 \theta_{3}\right)\left(-2 \theta_{2}+\theta_{5}\right)+\mathcal{O}\left(z_{i}\right) \\
& \mathcal{L}_{23}=-39\left(-2 \theta_{1}+\theta_{4}\right) \theta_{3}+13\left(-2 \theta_{1}+\theta_{4}\right)\left(-2 \theta_{2}+\theta_{5}\right) \\
& +13\left(-2 \theta_{1}+\theta_{4}\right)\left(\theta_{1}+\theta_{3}-\theta_{5}\right)-13 \theta_{1}\left(\theta_{1}+\theta_{3}-\theta_{5}\right)+\theta_{4}\left(\theta_{3}-\theta_{2}\right)-6 \theta_{4}\left(3 \theta_{2}-2 \theta_{3}\right) \\
& -8\left(\theta_{3}-\theta_{2}\right)\left(-2 \theta_{1}+\theta_{4}\right)-4\left(\theta_{1}-2 \theta_{4}+\theta_{5}\right)\left(\theta_{3}-\theta_{2}\right)+\mathcal{O}\left(z_{i}\right)
\end{aligned}
$$

The first 19 operators are obtained from the Mori cone (3.18), while the last four we 
got from the relation (3.21) and via the Dwork-Katz-Griffith's reduction scheme applied to $(3.23)$.

\begin{tabular}{|lllll|}
\hline$K_{J_{1} J_{1} J_{1}}=8$ & $K_{J_{1} J_{1} J_{2}}=16$ & $K_{J_{1} J_{2} J_{2}}=26$ & $K_{J_{2} J_{2} J_{2}}=38$ & $K_{J_{1} J_{1} J_{3}}=24$ \\
$K_{J_{1} J_{2} J_{3}}=39$ & $K_{J_{2} J_{2} J_{3}}=57$ & $K_{J_{1} J_{3} J_{3}}=58$ & $K_{J_{2} J_{3} J_{3}}=85$ & $K_{J_{3} J_{3} J_{3}}=125$ \\
$K_{J_{1} J_{1} J_{4}}=20$ & $K_{J_{1} J_{2} J_{4}}=34$ & $K_{J_{2} J_{2} J_{4}}=52$ & $K_{J_{1} J_{3} J_{4}}=51$ & $K_{J_{2} J_{3} J_{4}}=78$ \\
$K_{J_{3} J_{3} J_{4}}=116$ & $K_{J_{1} J_{4} J_{4}}=42$ & $K_{J_{2} J_{4} J_{4}}=68$ & $K_{J_{3} J_{4} J_{4}}=102$ & $K_{J_{4} J_{4} J_{4}}=84$ \\
$K_{J_{1} J_{1} J_{5}}=32$ & $K_{J_{1} J_{2} J_{5}}=52$ & $K_{J_{2} J_{2} J_{5}}=78$ & $K_{J_{1} J_{3} J_{5}}=78$ & $K_{J_{2} J_{3} J_{5}}=117$ \\
$K_{J_{3} J_{3} J_{5}}=174$ & $K_{J_{1} J_{4} J_{5}}=68$ & $K_{J_{2} J_{4} J_{5}}=104$ & $K_{J_{3} J_{4} J_{5}}=156$ & $K_{J_{4} J_{4} J_{5}}=136$ \\
$K_{J_{1} J_{5} J_{5}}=104$ & $K_{J_{2} J_{5} J_{5}}=156$ & $K_{J_{3} J_{5} J_{5}}=234$ & $K_{J_{4} J_{5} J_{5}}=208$ & $K_{J_{5} J_{5} J_{5}}=312$ \\
\hline
\end{tabular}

Table B.1: Intersection numbers for $\mathbf{P}^{4}(1,2,3,3,4)$.

\begin{tabular}{|rrrrrrrr|}
\hline$[0,0,0,1,0]$ & -2 & {$[0,0,1,0,0]$} & -2 & {$[1,0,0,1,0]$} & -2 & {$[1,0,0,0,0]$} & -2 \\
{$[0,0,0,0,1]$} & 3 & {$[0,0,0,1,1]$} & 3 & {$[1,0,0,1,1]$} & 3 & {$[1,1,1,1,1]$} & 3 \\
{$[0,1,1,0,0]$} & -2 & {$[0,1,1,0,1]$} & 3 & {$[0,1,1,1,1]$} & 3 & {$[0,1,1,1,2]$} & 7 \\
{$[0,1,2,0,0]$} & -2 & {$[0,1,2,0,1]$} & 3 & {$[0,1,2,1,1]$} & 3 & {$[0,1,2,1,2]$} & 7 \\
{$[0,2,3,0,0]$} & -4 & {$[0,2,3,0,1]$} & 9 & {$[0,2,3,0,2]$} & -6 & {$[0,2,3,0,3]$} & 1 \\
{$[0,2,3,1,1]$} & 9 & {$[0,2,3,1,2]$} & -18 & {$[0,2,3,1,3]$} & 9 & & \\
{$[0,2,3,2,2]$} & -6 & {$[0,2,3,2,3]$} & 9 & & & & \\
{$[0,3,4,0,0]$} & -6 & {$[0,3,4,0,1]$} & 15 & {$[0,3,4,0,2]$} & -12 & {$[0,3,4,0,3]$} & 3 \\
{$[0,3,4,1,1]$} & 15 & {$[0,3,4,1,2]$} & -36 & & & & \\
{$[0,3,5,0,0]$} & -6 & {$[0,3,5,0,1]$} & 15 & {$[0,3,5,0,2]$} & -12 & {$[0,3,5,1,1]$} & 15 \\
{$[0,4,5,0,0]$} & -8 & {$[0,4,5,0,1]$} & 21 & {$[0,4,6,0,0]$} & -32 & & \\
{$[1,1,1,1,2]$} & 7 & {$[1,1,1,2,2]$} & 7 & {$[1,1,1,2,3]$} & 7 & {$[1,1,2,1,1]$} & 3 \\
{$[1,1,2,1,2]$} & 7 & {$[1,1,2,2,2]$} & 7 & {$[1,1,2,2,3]$} & 8 & {$[1,1,3,2,3]$} & 3 \\
{$[1,2,2,2,3]$} & 7 & {$[1,2,3,1,1]$} & 9 & {$[1,2,3,1,2]$} & -18 & {$[1,2,3,1,3]$} & 9 \\
{$[1,2,3,2,2]$} & -18 & {$[1,3,4,1,1]$} & 15 & & & & \\
\hline
\end{tabular}

Table B.2: A list of the non-zero instanton numbers for rational curves of degree $\left[a_{1}, \ldots, a_{5}\right]$ on $\mathbf{P}^{4}(1,2,3,3,4)$, with the restriction $\sum_{i=1}^{5} a_{i} \leq 10$; the non-existence of the curve of degree $[0,1,0,0,0]$ is explained in section 4 . 


\section{References}

[1] S. Ferrara and A. Strominger, in the Proceedings of the Strings ' 89 workshop, College Stattion, 1989;

A. Strominger, Commun. Math. Phys. 133 (1990) 163;

P. Candelas and X. de la Ossa, Nucl. Phys. B355 (1991) 455.

[2] L. Dixon, V. Kaplunovsky and J. Louis, Nucl. Phys. B329 (1990) 27.

[3] W. Lerche, C. Vafa and N. Warner, Nucl. Phys. B324 (1989) 427.

[4] J. Distler and S. Kachru, Duality of $(0,2)$ String Vacua, hep-th/9501111.

[5] E. Witten, Mirror Manifolds And Topological Field Theory, in Essays on Mirror Manifolds, (ed. S.-T. Yau), Int. Press, Hong Kong, 1992 p. 120-158, hep-th/9112056.

[6] E.g. K. Kodaira, Complex Manifolds and Deformations of Complex Structure (Springer Verlag 1985).

[7] L. Dixon: in Superstrings, Unified Theories and Cosmology 1987, eds. G. Furlan et al. (World Scientific, Singapore, 1988) p. 67-127.

[8] M. Bershadsky, S. Cecotti, H. Ooguri and C. Vafa (with an appendix by S. Katz), Nucl. Phys. B405 (1993) 279, hep-th/9302103.

[9] V. Kaplunovsky and J. Louis, On Gauge Couplings in String Theory, hep-th/9502077.

[10] Seiberg and Witten, Nucl. Phys. B426 (1994) 19-52, Erratum-ibid. Nucl. Phys. B430 (1994) 485-486

[11] A. Klemm, W. Lerche, S. Theisen and S. Yankielowicz Phys. Lett. B 344 (1995) 169175, hep-th/9411048 and On The Monodromies Of $N=2$ Supersymmetric Yang-Mills Theory, hep-th/9412158, P. C. Argyres and A. E. Faraggi The Vacuum Structure and Spectrum of $N=2$ Supersymmetric $S U(n)$ Gauge Theory hep-th/9411057

[12] A. Klemm, W. Lerche, S. Theisen, Nonperturbative Effective Actions of $N=2 \mathrm{Su}-$ persymmetric Gauge Theories. CERN-TH-95-104 hep-th/9505150

[13] A. Ceresole, R. D'Auria, S. Ferrara, A. Van Proeyen, On Electromagnetic Duality In Locally Supersymmetric $N=2$ Yang-Mills Theory. CERN-TH-7510-94, hepth/9412200 and Duality Transformations in Supersymmetric Yang-Mills-Theories Coupled to Supergravity CERN-TH-7547-94, hep-th/9502072

[14] S. Kachru and C. Vafa, Exact Results for N=2 Compactifications of Heterotic Strings, hep-th/9505105.

[15] E. Martinec, Phys. Lett. 217B (1989) 431, C. Vafa and N. P. Warner, Phys. Lett. 218B (1989) 51.

[16] V. I. Arnol'd, V. A. Vasil'ev, V. V. Goryunov and O. V. Lyashko, Singularities Local and Global Theory in Dynamical Systems, Enc. Math. Sc. Vol 6, Ed. V. I. Arnold, (1991) Springer, Heidelberg.

[17] D. Gepner, Phys. Lett. 199B (1987) 380, Nucl. Phys. B296 (1988) 757, Nucl. Phys. B311 (1988) 191;

C. Vafa, Mod. Phys. Lett. A4 (1989) 1169. 
[18] P. Candelas, X. De la Ossa, P. Green and L. Parkes, Nucl. Phys. B359 (1991) 21.

[19] D. Morrison, Picard-Fuchs Equations and Mirror Maps for Hypersurfaces, in Essays on Mirror Manifolds, ed. S.-T. Yau, (Int. Press, Hong Kong, 1992), hep-th/9111025.

[20] A. Klemm and S. Theisen, Nucl. Phys. B389 (1993) 153, hep-th/9205041; Theor. Math. Phys. 95 (1993) 583, hep-th/9210142;

A. Font, Nucl. Phys. B391 (1993) 358, hep-th/9203084.

[21] P. Candelas, X. de la Ossa, A. Font, S. Katz and D. Morrison, Nucl. Phys. B416 (1994) 481, hep-th/9308083;

P. Candelas, A. Font, S. Katz and D. Morrison, Nucl. Phys. B429 (1994) 626, hepth/9403187.

[22] S. Hosono, A. Klemm, S. Theisen and S.-T. Yau: Mirror Symmetry, Mirror Map and Applications to Calabi-Yau Hypersurfaces, HUTMP-93/0801, LMU-TPW-93-22, to be published in Commun. Math. Phys., hep-th/9308122.

[23] D.R. Morrison and M.R. Plesser, Summing the Instantons: Quantum Cohomology and Mirror Symmetry in Toric Varieties, hep-th/9412236.

[24] B. Greene and R. Plesser, Nucl. Phys. B338 (1990) 15.

[25] A. Klemm and R. Schimmrigk, Nucl. Phys. B411 (1994) 559, hep-th/9204060;

M. Kreuzer and H. Skarke, Nucl. Phys. B388 (1993) 113, hep-th/9205004.

[26] V. Batyrev, Journal Alg. Geom. 3 (1994) 493, alg-geom/9310003.

[27] V. Batyrev, Duke Math. Journ., 69 (1993) 349

[28] S.-S. Roan: Int. J. Math 2 (1991) 439

[29] P.Berglund and S. Katz, Mirror Symmetry Constructions: A Review to appear in Essays of Mirror Manifolds II (Ed. S. T. Yau), hep-th/9406008.

[30] P. Candelas, X. de la Ossa and S. Katz: Mirror Symmetry for Calabi-Yau Hypersurfaces in Weighted $\mathbf{P}^{4}$ and an Extension of Landau-Ginzburg Theory, IASSNS-HEP94/100,NEIP-94-009, OSU-M-93-3, UTTG-25-93, hep-th/9412117.

[31] B. Greene and R. Plesser, in the Proceedings of PASCOS '91, Boston 1991, hepth/9110014.

[32] P. Berglund and T. Hübsch, Nucl. Phys. B393 (1993) 377, hep-th/9201014.

[33] R. Dijkgraaf, E. Verlinde, H. Verlinde and C. Vafa, Commun. Math. Phys. 123 (1989) 485.

[34] P. Berglund and M. Henningson, Nucl. Phys. B433 (1995) 311, hep-th/9401029; see also On the Elliptic Genus and Mirror Symmetry to appear in Essays of Mirror Symmetry II, eds. B. Greene and S.T. Yau, hep-th/9406045.

[35] M. Kreuzer, Phys. Lett. 328B (1994) 312, hep-th/9402114.

[36] D.R. Morrison and M.R. Plesser, work in progress.

[37] V. Batyrev and D. Cox, Duke Math. Journ. 74 (1994) 293, alg-geom/9306011.

[38] B. Dwork, Ann. of Math. (2) 80 (1964) 227.

[39] N. Katz, Publ. Math. I.H.E.S. 35 (1968) 71. 
[40] P. Griffiths, Ann. of Math. 90 (1969) 460.

[41] P.S. Aspinwall, B.R. Greene and D.R. Morrison, Nucl. Phys. B416 (1994) 414, hepth/9309097.

[42] T. Oda and H. S. Park, Tôhoku Math. J. 43 (1991) 375.

[43] D. Morrison, Amer. Math. Soc. 6 (1993) 223; Compactifications of Moduli spaces inspired by mirror symmetry DUK-M-93-06, llg-geom/9304007.

[44] I. M. Gel'fand, A. V. Zelevinkii and M. M. Kapranov, Functional Anal. Appl. 232 (1989) 12, English trans. 94.

[45] W. Lerche, D. J. Smit and N. P. Warner, Nucl. Phys. B372 (1992) 87, hep-th/9108013.

[46] S. Hosono, A. Klemm, S. Theisen and S.-T. Yau, Nucl. Phys. B433 (1995) 501, hepth/9406055.

[47] P. Berglund and S. Katz, Nucl. Phys. B420 (1994) 289, hep-th/9311014.

[48] R. Hartshorne, Algebraic Geometry, Grad. Texts in Mathematics 52, (Springer Verlag, Heidelberg 1977).

[49] P.S. Aspinwall and D.R. Morrison: Phys. Lett. 334B (1994) 79, hep-th/9406032.

[50] C. Vafa, A Stringy Test of the Fate of the Conifold, HUTP-95/A014, hep-th/9505023.

[51] E. Witten, Nucl. Phys. B403 (1993) 159, hep-th/9301042.

[52] J. De Loera, Triangulations of Polytopes and Computational Algebra, Ph.D. thesis, Cornell University, in preparation.

[53] S. Katz and S.A. Strømme, Schubert: a Maple package for intersection theory. Available by anonymous ftp from ftp.math.okstate.edu or linus.mi.uib.no, cd pub/schubert.

[54] D. Eisenbud and J. Harris, Proc. Symp. Pure Math. 46 Part I (1987) 3.

[55] A. Altman and S. Kleiman, Comp. Math. 34 (1977) 3.

[56] M. Kontsevich, Enumeration of Rational Curves via Torus Actions, alg-geom/9405035.

[57] P.M.H. Wilson, Inv. Math. 107 (1992) 561.

[58] M. Kreuzer and H. Starke, Nucl. Phys. B405 (1993) 305, hep-th/9211047 and hepth/9412033; A. Niemeier, Klassifizierung von Calabi-Yau-Stringkompaktifizierungen durch $N=2$-superkonforme LG-Theorien, Diploma Thesis, TU-Munich (1993)

[59] P. Candelas, A. Font, S. Katz and D. Morrison, Nucl. Phys. B429 (1994) 626, hepth/9403187.

[60] B.R. Greene, D.R. Morrison and A. Strominger, Black Hole Condensation and the Unification of String Vacua, hep-th/9504145.

[61] A. Strominger, Massless Black Holes and Conifolds in String Theory, hep-th/9504090.

[62] S. Ferrara, J. A. Harvey, A. Strominger and C. Vafa, Second-Quantized Mirror Symmetry, hep-th/9505162. 